\title{
On hypervigilance and extreme evaluations in Borderline Personality Disorder
}

Citation for published version (APA):

Sieswerda, S. H. (2009). On hypervigilance and extreme evaluations in Borderline Personality Disorder. [Doctoral Thesis, Maastricht University]. Datawyse / Universitaire Pers Maastricht. https://doi.org/10.26481/dis.20090514ss

Document status and date:

Published: 01/01/2009

DOI:

10.26481/dis.20090514ss

Document Version:

Publisher's PDF, also known as Version of record

\section{Please check the document version of this publication:}

- A submitted manuscript is the version of the article upon submission and before peer-review. There can be important differences between the submitted version and the official published version of record.

People interested in the research are advised to contact the author for the final version of the publication, or visit the DOI to the publisher's website.

- The final author version and the galley proof are versions of the publication after peer review.

- The final published version features the final layout of the paper including the volume, issue and page numbers.

Link to publication

\footnotetext{
General rights rights.

- You may freely distribute the URL identifying the publication in the public portal. please follow below link for the End User Agreement:

www.umlib.nl/taverne-license

Take down policy

If you believe that this document breaches copyright please contact us at:

repository@maastrichtuniversity.nl

providing details and we will investigate your claim.
}

Copyright and moral rights for the publications made accessible in the public portal are retained by the authors and/or other copyright owners and it is a condition of accessing publications that users recognise and abide by the legal requirements associated with these

- Users may download and print one copy of any publication from the public portal for the purpose of private study or research.

- You may not further distribute the material or use it for any profit-making activity or commercial gain

If the publication is distributed under the terms of Article $25 \mathrm{fa}$ of the Dutch Copyright Act, indicated by the "Taverne" license above, 


\section{On Hypervigilance and Extreme Evaluations in Borderline Personality Disorder}

Simkje Sieswerda 
(C) Simkje Sieswerda, Darmstadt 2009

Cover drawing:

Anna Higgie, future03

Cover design:

Datawyse \& Simkje Sieswerda

ISBN 9789052788333

Universitaire Pers Maastricht 


\title{
On Hypervigilance and Extreme Evaluations in Borderline Personality Disorder
}

\author{
PROEFSCHRIFT \\ ter verkrijging van de graad van doctor aan de Universiteit Maastricht, \\ op gezag van de Rector Magnificus, Prof. mr. G.P.M.F. Mols, \\ volgens het besluit van het College van Decanen, \\ in het openbaar te verdedigen op \\ donderdag 14 mei 2009 om 16.00 uur \\ door
}

Simkje Heleen Sieswerda

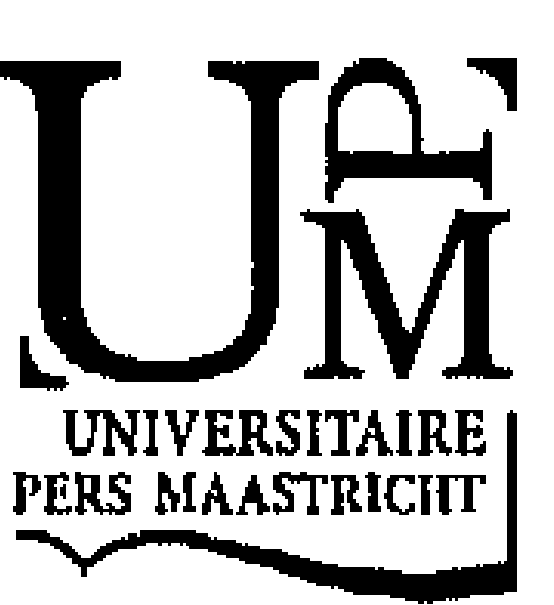




\section{Promotor}

Prof. dr. A. Arntz

\section{Beoordelingscommissie}

Prof. dr. M. Peters (voorzitter)

Dr. D. Bernstein

Prof. dr. S. Herpertz

Prof. dr. M. Kindt

Prof. dr. C. de Ruiter 
Wat het eerst opvalt is het bijzondere aan hem: die ogen, jukbeenderen en huid die anders zijn, onderscheiden hem en herinneren ons eraan dat daar iemand staat. Het afwijkende van dat gezicht onthult ten volle wat ieder gezicht bij aandachtige beschouwing zou moeten onthullen: dat gewoon bij de mens niet bestaat.

- Julia Kristeva, De vreemdeling in onszelf (1991) 


\section{Contents}

1 Hypervigilance and Extreme Evaluations in Borderline Personality Disorder: General Introduction 9

Introduction ................... 10

Conceptual development of borderline pathology . . . . . . . 10

Characteristics of borderline pathology . . . . . . . . . . . 11

Hypervigilance in borderline patients . . . . . . . . . . . 14

Extreme evaluations in borderline patients . . . . . . . . . . 16

Research questions and organization of this thesis . . . . . . 17

2 Hypervigilance in Patients with Borderline Personality Disorder: Speci$\begin{array}{lr}\text { ficity, Automaticity, and Predictors } & 19\end{array}$

Abstract . . . . . . . . . . . . . . . 19

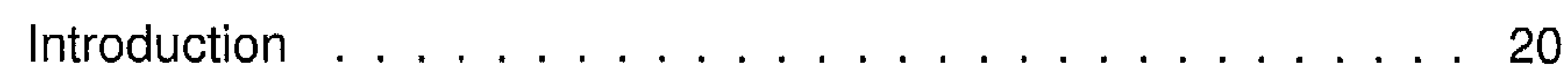

Method . . . . . . . . . . . . . . . . . . . 22

Results . . . . . . . . . . . . . . . . 28

Discussion . . . . . . . . . . . . . . 36

3 Successful Psychotherapy Reduces Hypervigilance in Borderline Personality Disorder $\quad 39$

Abstract . . . . . . . . . . . . . . . 39

Introduction . . . . . . . . . . . . 40

Method . . . . . . . . . . . . . . . . . . . 42

Results . . . . . . . . . . . . . . . . . 49 49

Discussion . . . . . . . . . . . . . . . . 54

4 Not Dichotomous, but Negative Interpersonal Evaluations Characterize Borderline Patients $\quad 59$

Abstract . . . . . . . . . . . . . . . 5 59 
Introduction .................... 60

Method . . . . . . . . . . . . . . . . . . . . . 6 64

Results ....................... . . . 71

Discussion . . . . . . . . . . . . . . . . 76

5 Negative Evaluations of Emotional Non-Interpersonal Situations Char$\begin{array}{ll}\text { acterize Borderline Personality Disorder } & \mathbf{8 1}\end{array}$

Abstract . . . . . . . . . . . . . . . 81

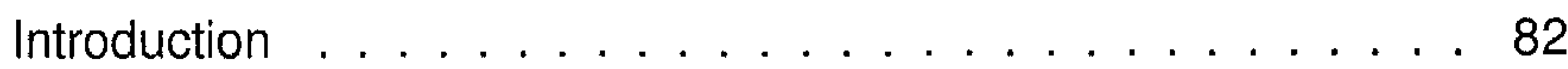

Method . . . . . . . . . . . . . . . . . . . 84

Results . . . . . . . . . . . . . . . . 89

Discussion . . . . . . . . . . . . . . 96

6 Change of Extreme and Negative Interpersonal Evaluations in Borderline Patients During Psychotherapy $\quad 99$

Abstract . . . . . . . . . . . . . . . . 99

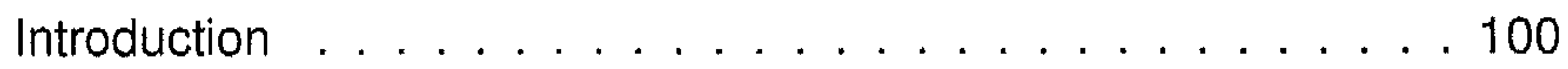

Method . . . . . . . . . . . . . . . . . . 102

Results . . . . . . . . . . . . . . . . . . 109

Discussion . . . . . . . . . . . . . . . . . 114

7 Hypervigilance and Extreme Evaluations in Borderline Personality

Disorder: General Discussion 119

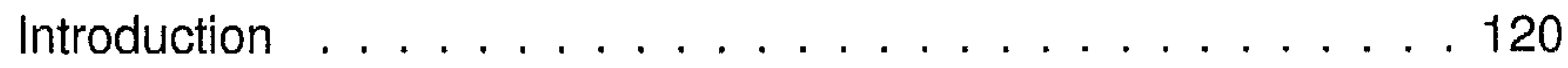

Summary of the findings . . . . . . . . . . . . . 120

Discussion of the findings . . . . . . . . . . . . . 122

Some limitations and other methodological considerations . . . 132

Closing remark . . . . . . . . . . . . . . . . . 139

$\begin{array}{ll}\text { Bibliography } & 141\end{array}$

$\begin{array}{ll}\text { Samenvatting } & 161\end{array}$

$\begin{array}{ll}\text { Dankwoord } & 171\end{array}$

$\begin{array}{ll}\text { Curriculum Vitae } & 175\end{array}$ 


\section{Hypervigilance and Extreme \\ Evaluations in Borderline Personality Disorder:}

General Introduction 
CHAPTER 1

\section{Introduction}

Since its differentiation from schizophrenia in the beginning of the twentieth century, the pathology associated with borderline personality disorder (BPD) has puzzled clinicians and researchers alike. Patients in this condition generally have intact reality testing, but their suffering does not seem less intense than that of schizophrenic patients. In order to get grip on this affliction, many different conceptualizations and hypotheses have been formulated; a process, in which the generation of theories not always has been balanced by the empirical testing of these.

This thesis aims to contribute to the understanding of BPD by presenting empirical research on two phenomena. The first phenomenon is hypervigilance, the second is extreme evaluations. According to prevailing theories, hypervigilance and extreme evaluations are both prominent in BPD. Five experiments will test this prominence, test hypotheses explaining these phenomena, and elaborate on the impact of the findings.

In this introductory chapter the conceptual development of borderline pathology is summarized, followed by an overview of the current diagnostic criteria and some other important characteristics. Subsequently, the concepts of hypervigilance and extreme evaluations are introduced including related hypotheses. A summary of the research questions and presentation of the thesis organization closes this introduction.

\section{Conceptual development of borderline pathology}

$\mathrm{BPD}$, as it is known today, has been distinguished and conceptualized in various ways during the past century. Originally, the concept of 'borderline' pathology was used by psychiatrists to describe phenomena on the borderline between schizophrenia and normality. Patients who were clearly disturbed but did not fulfill the criteria for schizophrenia were conceived of as borderline or latent cases of schizophrenia (e.g., Ingenhoven \& van den Brink, 1994; Kroll, 1988).

The label was subsequently adopted by psychoanalysts (e.g., Stern, 1938) for patients showing poor effects in classical psychoanalysis. These patients were conceived of as being on the verge of a mature 'neurotic' and primitive 'psychotic' personality. A disruption in a very early ('separation-individuation') developmental phase as the main cause for the 'borderline personality organization' (BPO) as hypothesized by Kernberg (1967) became a very influential 
idea.

Common from 1980 onwards has been the descriptive eclectic concept of 'borderline personality disorder' (BPD) of the Diagnostic and Statistical Manual of Mental Disorders (DSM) (American Psychiatric Association, 1980, 1987, 1994, 2000). The 'emotional instable personality disorder, borderline type' which later appeared in the International Classification of Diseases (ICD-10; World Health Organization, 1991, 1993) is broadly similar to BPD. The DSM concept resulted from a political and economical compromise (Kroll, 1988) or a 'double parenthood' (Rohde-Dachser, 1986) of psychiatrists and psychoanalysts. The DSM reformulated the difficult psychodynamic description into a set of observable criteria, adopted the psychoanalytical idea of a disordered personality with an emphasis on affective and relational instability, and largely excluded disturbed (schizophrenic) cognitions which were moved to the schizotypical personality disorder.

The introduction of the BPD criteria in the DSM-III (American Psychiatric Association, 1980) greatly increased empirical research on BPD. Studies on psychotic and depressive symptomatology soon casted doubt on the idea of BPD being a schizophrenic or affective spectrum disorder (e.g., Ingenhoven \& van den Brink, 1994). The idea of BPD resulting from a disturbance in a single primitive developmental phase neither found clear empirical support (e.g., Westen, 1990). Behavioral and cognitive psychology has long been reluctant to acknowledge the existence of personality and focused more on transdiagnostic phenomena rather than on disorders per se (see also, Linehan, 1993). Cognitive-behavioral models of BPD, consisting of specific complexes of cognitive schemata, erratic cognitions, deficit coping skills, and/or temperamental factors (e.g., Pretzer, 1990; Linehan, 1993), have therefore been formulated only recently and are currently being put to the test.

\section{Characteristics of borderline pathology}

This thesis focuses on the clear and widely accepted concept of BPD as described by the latest DSM-criteria (American Psychiatric Association, 2000). These criteria are presented in Table 1.1. BPO, the concept for borderline pathology of Kernberg (1975) whose hypotheses on extreme evaluations will be tested as well, is summarized in Table 1.2. The concepts of BPD and BPO cannot be equated. Only about $40 \%$ of patients with a BPO have been found to meet the criteria for BPD (Pretzer, 1990). Patients with BPD probably form a 


\section{CHAPTER 1}

Table 1.1: The diagnostic criteria for Borderline Personality Disorder according to the DSM-IV (American Psychiatric Association, 2000).

(I) An enduring pattern of inner experience and behavior that deviates markedly from the expectations of the individual's culture, and which is inflexible, pervasive, pathological, persistent, not better accounted for by another mental disorder, nor due to direct physiological effects of a substance or a general medical condition. ${ }^{1}$

(II) A pervasive pattern of instability of interpersonal relationships, selfimage, and affects, and marked impulsivity beginning by early adulthood and present in a variety of contexts, as indicated by five (or more) of the following:

1. Frantic efforts to avoid real or imagined abandonment. Note: Do not include suicidal or self-mutilating behavior covered in Criterion 5.

2. A pattern of unstable and intense interpersonal relationships characterized by alternating between extremes of idealization and devaluation.

3. Identity disturbance: markedly and persistently unstable selfimage or sense of self.

4. Impulsivity in at least two areas that are potentially self-damaging (e.g., spending, sex, substance abuse, reckless driving, binge eating). Note: Do not include suicidal or self-mutilating behavior covered in Criterion 5.

5. Recurrent suicidal behavior, gestures, or threats, or self-mutilating behavior.

6. Affective instability due to a marked reactivity of mood (e.g., intense episodic dysphoria, irritability, or anxiety usually lasting a few hours and only rarely more than a few days.

7. Chronic feelings of emptiness.

8. Inappropriate, intense anger or difficulty controlling anger (e.g., frequent displays of temper, constant anger, recurrent physical fights).

9. Transient, stress-related paranoid ideation or severe dissociative symptoms.

\footnotetext{
${ }^{1}$ Summary of the general criteria for a Personality Disorder.
} 
GENERAL INTRODUCTION

Table 1.2: The criteria for Borderline Personality Organization formulated by Kernberg (1975) and summarized by Gabbard (1994).

(I) Nonspecific manifestations of ego weakness ${ }^{1}$

1. Lack of anxiety tolerance

2. Lack of impulse control

3. Lack of developed subliminatory channels ${ }^{2}$

(II) Shift toward primary-process thinking ${ }^{3}$

(III) Specific defensive operations characteristic of borderline personality organization

1. Splitting ${ }^{4}$ and splitting tendencies, including primitive idealization, omnipotence and devaluation

2. Early forms of projection ${ }^{5}$, especially projective identification ${ }^{6}$

(IV) Pathological internalized object relations ${ }^{7}$

(V) Generally intact reality testing

\footnotetext{
1 Weakness of executive functioning, for example, difficulty with modulating affects, delaying discharge of impulses, using conscience to guide behavior, and sublimating drives. ${ }^{2}$ Socially accepted behaviors to express potentially maladaptive feelings or impulses (e.g., contact sports to channel angry impulses) (American Psychiatric Association, 2000). ${ }^{3}$ Psychotic-like thinking in the absence of structure or in the presence of strong affects. ${ }^{4}$ Compartmentalization of opposite affect states and failure to integrate the positive and negative qualities of the self and/or others into cohesive images (American Psychiatric Association, 2000). ${ }^{5}$ False attribution of own unacceptable feelings, impulses, or thoughts to another (American Psychiatric Association, 2000). ${ }^{6}$ Projection in which the individual does not fully disavow what is projected, but misattributes own affects and impulses as justifiable reactions to the other person (American Psychiatric Association, 2000). ${ }^{7}$ Maladaptive representations of relations with significant others.
} 


\section{CHAPTER 1}

subset of patients with BPO.

BPD is a massive burden for patients, their families, friends, health centers, and for society as a whole. The disorder is very common, affecting about 1 to $2 \%$ of the general population (American Psychiatric Association, 1994; Samuels et al., 2002; Torgersen, Kringlen, \& Cramer, 2001), 10\% of outpatients, $20 \%$ among psychiatric inpatients, and $30-60 \%$ among patients receiving treatments for their personality disorder (PD) (American Psychiatric Association, 2000). BPD has further been identified in both genders and in several cultures around the world, although patients with BPD are predominantly female (about 70 to $75 \%$ (American Psychiatric Association, 2000; Paris, 2004; Widinger \& Weissman, 1991)) and the infliction may be rather rare in some nonwestern countries (e.g., Kenya; Loranger et al., 1994). The seriousness of the symptomatology can be illustrated by a suicide risk of about 10\% (Paris, 1993), high prevalence of subjective dysphoric states (extreme feelings, destructiveness, fragmentation and victimization) compared to patients with other personality disorders (25-40\% vs. $10 \%$; Zanarini et al., 1998a), and a dramatic quality of life reduction (EQ-5D utility of 0.48 ; van Asselt, Dirksen, \& Arntz, 2008) compared to persons with other health problems (e.g., EQ-5D utilities of 0.79 or 0.66 for persons with respectively asthma or low back pain) and the general population (EQ-5D utility of 0.84 ; e.g., Bürström, Johannesson, \& Diderichsen, 2001).

\section{Hypervigilance in borderline patients}

Hypervigilance can be described as an anxious state, in which an individual engages in selective attention to threatening stimuli in the environment, particularly to those which have been associated with threat before. In this mode, initial automatic encoding of threat stimuli is prioritized at the expense of strategic rehearsal of the material for encoding into memory. An analogue is the physiological response to threat, involving blood being pumped to the muscles to prepare them for action, but away from the digestive system (Williams, Watts, MacLeod, \& Mathews, 1997). Several authors suppose that much of the behavior of patients with BPD stems from fear, panic, hopelessness and desperation, and directly or indirectly state that hypervigilance plays a central role in BPD. 


\section{Beckian model}

Models of BPD along the lines of (e.g., Beck, 1976) hypothesize that patients with this disorder process information through a specific set of schemas or core beliefs of themselves and others, such as, 'I am powerless and vulnerable', 'I am inherently unacceptable', and 'Others are or the world is dangerous and malevolent' (Pretzer, 1990). When activated, these schemas and their incompatibility, i.e., the belief of needing support in a dangerous world but not trusting others at the same time, are thought to generate excessive fear and a state of hypervigilance in patients with BPD (Pretzer, 1990).

\section{Trauma model}

Histories of childhood trauma in many borderline patients (Herman, Perry, \& van der Kolk, 1989; Sabo, 1997; Zanarini, 1997) have led several investigators to look for an overlap between posttraumatic stress disorder (PTSD) and BPD (Gunderson \& Sabo, 1993; Herman et al., 1989). Recurrent anxious states, feelings of emptiness, dissociative experiences, outbursts of anger and hypervigilance in BPD might result from traumatic experience similarly as in PTSD; other characteristics of BPD such as a distrustful attitude toward the world, identity problems, self-mutulating and suicidal behaviours and psychosomatic symptoms bear resemblance with those of the diagnosis of enduring personality change after catastrophic experience (World Health Organization, 1993) or 'complex PTSD' (e.g., Herman, 1992) and may have been brought about by chronic traumatic experiences. Behavioral theories hypothesize that traumatic experiences can result in chronic anxiety through classical and secondary conditioning (e.g., Joseph, Williams, \& Yule, 1997). According to cognitive theories a traumatic event can create a disparity between trauma-related information and existing cognitive schemas (e.g., Horowitz, 1993; Janoff-Bulman, 1991; Foa, Steketee, \& Rothbaum, 1989) causing strategic avoidance of trauma-related stimuli as well as an automatic bias towards threatening stimuli.

\section{Emotional dysregulation model}

The cognitive behavioral model of Linehan (1993) conceives emotional dysregulation as being the hallmark of BPD. This emotional dysregulation is thought of as being due to high emotional vulnerability plus an inability to regulate emotions, having their roots in a biological predisposed emotional temperament 


\section{CHAPTER 1}

exacerbated by specific environmental experiences. Besides high emotional intensity and slow return to emotional baseline, emotional vulnerability also includes high sensitivity or hypervigilance to emotional stimuli. However, different than the trauma and Beckian model, this model hypothesizes a high sensitivity for emotional stimuli in general, also not necessarily for emotionally negative stimuli or stimuli related to certain schemata.

\section{Extreme evaluations in borderline patients}

Extreme evaluations are thought to be a central characteristic of BPD in several models. These evaluations are thought to cause or at the least perpetuate the unstable and extreme affects and behaviors of patients with BPD.

\section{Beckian models}

Beckian models suppose that two kinds of extreme evaluations are central in BPD: dichotomous thinking and schema congruent evaluations (Pretzer, 1990). Pretzer (1990, p. 187) describes dichotomous thinking as:

"the tendency to evaluate experiences in terms of mutually exclusive categories (e.g., good or bad, success or failure, trustworthy or deceitful) rather than seeing experiences as falling along continua".

Beck (1976, p. 93) similarly states:

"Events are labeled as black or white, good or bad, wonderful or horrible. The basic premises underlying this kind of thinking are generally couched in absolute terms such as "always" or "never"."

Lacking intermediate evaluative categories, dichotomous evaluation is associated with both extreme as well as unstable affects and behaviors. Although dichotomous thinking is seen as a common cognitive distortion, which may also be displayed by other patients, it is thought to be highly prominent in BPD. Some theorists conceptualize dichotomous thinking in BPD as a regression to a lower, preoperational, cognitive developmental level, characterized by an inability to make stepwise gradations (Layden, Newman, Freeman, \& Morse, 1993). This regression would take place when maladaptive schemas are activated.

A second kind of extreme evaluations in Beckian models for BPD are schema congruent evaluations. As described in the previous section, these models 
(Layden et al., 1993; Pretzer, 1990) suppose that borderline patients tend to see themselves as extremely powerless and inherently unacceptable, and others or the world as dangerous and malevolent. The incompatible tendencies resulting from these schema's (approaching and avoiding others) are thought to further destabilize borderline patients.

\section{Object relational model of Kernberg}

The psychoanalytical object relational model of Kernberg (1996) conceives extreme 'black-or-white' thinking as 'splitting': a very specific defense mechanism which is assumed to be the hallmark of patients with a BPO, including practically all patients with BPD. Patients with a BPO are thought being stuck on a cognitive-emotional developmental level of three year olds. At this 'pre-oedipal' level, the integration of good and bad aspects of self- and other-representations is hypothesized to provoke massive anxiety because of (unconscious) fears that integration leads to annihilation of the good representations by the bad representations. Patients with BPO would therefore split good and bad aspects of themselves and others, resulting in extreme and unstable, unidimensional ('all bad' or 'all good') interpersonal evaluations, affects, and behaviors.

\section{Negative thinking}

Unstable and extreme affects and behaviors in BPD might finally stem from negative thinking. This hypothesis was put forward in many empirical studies finding that a strong and pervasive negativity bias was the most specific feature of borderline patients. A model with negative thinking as central characteristic of BPD has however not been articulated yet.

\section{Research questions and organization of this thesis}

This thesis aims investigating the hypothesized prominent roles of hypervigilance and extreme evaluations in BPD. In includes research focusing on the following questions.

- Is BPD characterized by hypervigilance and extreme evaluations? In other words, do patients with BPD show hypervigilance and extreme evaluations, and are these characteristics specific for BPD? 


\section{CHAPTER 1}

- What kind of hypervigilance and extreme evaluations are characteristic of BPD? For example, do patients with BPD show hypervigilance and extreme evaluations with regard to certain stimuli types such as schemarelated stimuli, emotionally negative stimuli in general, and/or interpersonal stimuli? Do these patients only show totally split, unidimensional extreme evaluations?

- What are potential predictors of hypervigilance and extreme evaluations in BPD?

- Is successful treatment of BPD related to decrease of hypervigilance and extreme evaluations?

The organization of this thesis is as follows. Chapters 2 and 3 focus on the the research questions with regard to hypervigilance. These chapters present experimental studies investigating whether and what kind of hypervigilance is characteristic of BPD (chapter 2), and whether this can be successfully treated (chapter 3). The next three chapters concentrate on extreme evaluations. Experimental studies in these chapters examined whether and what kind of extreme evaluations are characteristic of BPD in an interpersonal context (chapter 4) and a non-interpersonal context (chapter 5), and researched changes in these interpersonal evaluations during therapy (chapter 6). The thesis will end with a general discussion of the findings (chapter 7 ). 


\title{
Chapter 2
}

\section{Hypervigilance in Patients with Borderline Personality Disorder: Specificity, Automaticity, and Predictors ${ }^{1}$}

\begin{abstract}
According to cognitive theory, an important factor in borderline personality disorder (BPD) is hypervigilance. The aim of the present study was to test whether BPD patients show schema related biases, and to explore relations with childhood trauma, schemas, and BPD symptoms. Sixteen BPD patients were compared with 18 patients with a cluster $C$ personality disorder, 16 patients with an axis I disorder, and 16 normal controls. An emotional Stroop task was applied with schema related and unrelated, negative and positive, supra- and subliminal person related stimuli. BPD patients showed hypervigilance for both negative and positive cues, but were specifically biased towards schema related negative cues. Predictors were sexual childhood traumas and BPD related anxiety and identity disturbance symptoms. Both BPD and axis I disorder patients showed a trend for a bias for negative schema related subliminal stimuli. More attention to hypervigilance in BPD is recommended for clinical practice.
\end{abstract}

\footnotetext{
${ }^{1}$ The content of this chapter has been published as Sieswerda, S., Arntz, A., Mertens, I., and Vertommen, S. (2006), Hypervigilance in patients with porderline personality disorder: Specificity, automaticity, and predictors, Behaviour Research and Therapy, 45, 1011-1024.
} 


\section{CHAPTER 2}

\section{Introduction}

According to the Beckian cognitive model of borderline personality disorder (BPD), an important factor in the development and maintenance of this disorder is cognitive bias (Arntz, 2004; Pretzer, 1990). The model hypothesizes that BPD patients process information through a specific set of three core beliefs or schemas of themselves and others, i.e., 'I am powerless and vulnerable', 'I am inherently unacceptable', and 'Others are dangerous and malevolent'. Needing support in a dangerous world but not trusting others brings BPD patients in a state of hypervigilance. Schema specific information is highly prioritized or difficult to inhibit in this state, resulting in biases in early information processing phases such as selective attention.

Selective attention has been studied extensively in various disorders, and has been shown to play a crucial role in the etiology and maintenance of pathological anxiety in particular (Kindt \& Van den Hout, 2001; Williams et al., 1997). In BPD however, anxiety in general and early biases in particular, have not been paid much attention to by researchers. Physiological affective hyperarousal in BPD has been investigated more often (e.g., Herpertz et al., 2000, 2001a, 2001b; Schmahl et al., 2003), however with contradictory findings, i.e., from hypo-arousal to hyperarousal. The scarcity of selective attention studies of $\mathrm{BPD}$ is in contrast with the acknowledgement of anxiety as a significant aspect of BPD already in the earliest papers on 'borderline patients' (Hoch \& Cattell, 1959; Stern, 1938), the relation of BPD with childhood trauma (Herman et al., 1989; Sabo, 1997; Zanarini, 1997), and the relatively high comorbidity of BPD both with anxiety disorders (Zanarini et al., 1998b; Zimmerman \& Mattia, 1999) and anxious cluster personality disorders (PDs) (Zanarini et al., 1998c).

Diverging hypotheses exist about the specificity of early biases in BPD. BPD is often conceptualized as a post-trauma disorder (Gunderson \& Sabo, 1993; Herman et al., 1989), a view that is supported by data on high prevalences of interpersonal childhood traumas in BPD (Herman et al., 1989; Sabo, 1997; Zanarini, 1997). Cognitive-behavioral theories conceptualize these traumas as learning experiences resulting in specific trauma related cognitive schemas. These schemas facilitate but also bias information processing (Arntz, 2004; Pretzer, 1990), or result in relatively isolated memory structures generating pathological fear behaviors and cognitions (Foa et al., 1989). Both views hypothesize specific trauma related biases in BPD, like these have also been found in patients with post-traumatic stress disorder (PTSD) (McNally, 1998). 
Other theories on BPD hypothesize structural deficits causing general handicaps in emotional functioning instead of specific biases. Amongst them are Linehan's dialectical model (Linehan, 1993) assuming a general emotional dysregulation, and Kernberg's psychoanalytic theory (e.g., Kernberg, 1967) assuming immature cognitive-emotional functioning in these patients. Studies focusing on biological deficits such as serotonin dysregulation (Coccaro, 1989), limbic system irritability (Andrulonis et al., 1981), or attentional network deficits (Posner et al., 2002) also seem to take this stand.

Previous studies have found evidence for selective attention in BPD but did not resolve the stimulus specificity issue. Waller and Button (1999) investigated emotional Stroop interferences of BPD patients, patients with an anxiety or depressive disorder, and nonpatient controls. They found specific biases in BPD for stimuli related to self-criticism (e.g., failure), but not for stimuli related to criticism by others (e.g., ridiculed). A PD control group was not assessed, so it could not be tested whether the effect was specific for BPD or for PDs in general. Arntz, Appels, and Sieswerda (2000) who also applied an emotional Stroop task did compare BPD patients to other PD patients. They found a bias for emotionally negative stimuli related and unrelated to the BPD schemas in both BPD and cluster C PD (CPD) patients. Bias for emotionally positive stimuli was not investigated, leaving the question unanswered whether BPD patients show a really general emotional bias. The present study, that included control groups for both axis I and II, as well as emotionally positive stimuli, might clarify the specificity issue further. We hypothesized that patients with BPD would show deviating strong attentional biases, in particular for schema related stimuli.

A second unresolved issue is selective attention in BPD for not consciously perceived, i.e., subliminal stimuli. Schemas characteristically operate unconsciously and automatically (Beck, 1976), therefore they can be expected to be triggered by subliminal as well as supraliminal stimuli (Williams et al., 1997). Some studies even suggest that early bias for subliminal threats is a better predictor for emotional vulnerability than early bias for supraliminal threats (MacLeod \& Hagan, 1992; van den Hout, Tenney, Huygens, \& Merckelbach, 1995; Verhaak, Smeenk, van Minnen, \& Kraaimaat, 2004). Biases of subliminal stimuli in BPD were not found previously (Arntz et al., 2000) but this might be explained by methodological artifacts, like too short presentation times, no priming by supraliminal stimuli, and too small sample size. The present study applied less stringent calibration resulting in longer presentation times, presented the 
sub- and supraliminal stimuli mixed instead of blocked, and included more participants. Our hypothesis was that the interference of schema related subliminal stimuli of patients with BPD would be larger than we would find for the whole group.

A final objective of the present study was to explore potential predictors and consequences of hypervigilance in BPD. We investigated the relation between cognitive bias on the one hand and childhood trauma, BPD schemas, and BPD symptoms on the other hand. The symptom clusters we focused on were disinhibition and negative affectivity (anxiety in particular), which are personality traits characteristic for patients with BPD (Trull, 2001), and identity disturbance, which is another generally acknowledged problem in BPD (e.g., Pretzer, 1990; Kernberg, 1967).

\section{Method}

\section{Participants}

The study included 16 BPD patients, 18 CPD patients, and 16 patients with an axis I disorder (AID), who had been referred to outpatient community mental health services or inpatient mental hospitals in the Netherlands and Belgium. Sixteen nonpatient controls (NP) participated from the same regions. Ss had to be between 18 and 60 years of age. BPD, CPD and AID patients had to meet DSM-IV criteria for BPD, one or more CPD, or one or more AID, respectively. The NPs had to have no current axis I or axis II disorder and maximally one axis I disorder in complete remission. Control groups were matched to the BPD patients on age and sex. Control Ss meeting more than two BPD DSMIV criteria were not included. Ss having a bipolar or psychotic disorder, Ss being mentally retarded, visually handicapped, intoxicated by alcohol or drugs, or not having Dutch as mother tongue, were also excluded. Demographic and diagnostic variables of the four groups are presented in Table 2.1. The BPD group had more PD disorders and reported more severe BPD symptoms than the control groups. Across all patient groups, comorbidity was the rule rather than the exception.

\section{Design}

The study had a cross-sectional, mixed between-within design. Independent variables of the study were: Group (BPD, CPD, AID, NP), Stimulus Type (three 
HYPERVIGILANCE IN BPD: SPECIFICITY, AUTOMATICITY, AND PREDICTORS

Table 2.1: Demographic and diagnostic characteristics per group ${ }^{\perp}$.

\begin{tabular}{|c|c|c|c|c|}
\hline \multirow{3}{*}{ Variable } & \multicolumn{4}{|c|}{ Group } \\
\hline & BPD & CPD & AID & NP \\
\hline & $(n=16)$ & $(n=18)$ & $(n=16)$ & $(n=16)$ \\
\hline M Age & $27(6.8)$ & $29(9.2)$ & $30(9.6)$ & $26(5.4)$ \\
\hline$\%$ Female ${ }^{2}$ & $94(15)$ & $89(16)$ & $75(12)$ & $88(14)$ \\
\hline \multicolumn{5}{|l|}{ Education } \\
\hline$\%$ low - middle level ${ }^{3}$ & $44(7)$ & $39(7)$ & $56(9)$ & $56(9)$ \\
\hline$\%$ middle - high level ${ }^{4}$ & $56(9)$ & $61(11)$ & $44(7)$ & $44(7)$ \\
\hline \multicolumn{5}{|l|}{ Current employment $^{2}$} \\
\hline$\%$ employed & $50(8)$ & $39(7)$ & $31(5)$ & $50(8)$ \\
\hline$\%$ student & $25(4)$ & $33(6)$ & $19(3)$ & $44(7)$ \\
\hline$\%$ unemployed & $25(4)$ & $28(5)$ & $50(8)$ & $6(1)$ \\
\hline$\%$ Married/cohabiting & $13(2)$ & $44(8)$ & $38(6)$ & $50(8)$ \\
\hline \multicolumn{5}{|l|}{ Axis I disorders (SCID-I) ${ }^{5}$} \\
\hline$\% \operatorname{mood}$ & $44(7)$ & $50(9)$ & $13(2)$ & \\
\hline$\%$ anxiety & $63(10)$ & $67(12)$ & $94(15)$ & \\
\hline$\%$ somatoform ${ }^{2}$ & $0(0)$ & $6(1)$ & $19(3)$ & \\
\hline$\%$ eating ${ }^{2}$ & $19(3)$ & $11(2)$ & $0(0)$ & \\
\hline$\%$ impulse $^{2}$ & $6(1)$ & $0(0)$ & $0(0)$ & \\
\hline \# current disorders & $1.63(1.31)^{a}$ & $1.67(1.14)^{a}$ & $1.81(.98)^{a}$ & \\
\hline \# disorders in remission*** & $1.44(1.09)^{a}$ & $.83(.86)^{a, b}$ & $.50(1.03)^{b, c}$ & $.19(.40)^{c}$ \\
\hline \multicolumn{5}{|l|}{ PD disorders (SCID-II) } \\
\hline$\%$ paranoid $^{2}$ & $6(1)$ & $0(0)$ & & \\
\hline$\%$ borderline ${ }^{* * *}$ & $100(16)$ & $0(0)$ & & \\
\hline$\%$ avoidant & $25(4)$ & $56(10)$ & & \\
\hline$\%$ dependent $^{2}$ & $0(0)$ & $11(2)$ & & \\
\hline$\%$ obsessive-compulsive & $25(4)$ & $39(7)$ & & \\
\hline \# PD disorders* & $1.56(0.81)$ & $1.06(0.24)$ & & \\
\hline
\end{tabular}

${ }^{1}$ Standard deviations or frequencies are between parentheses. ${ }^{2}$ Low cell frequency did not allow testing. ${ }^{3}$ From no education up to and including vocational or technical training $<18$ years. ${ }^{4}$ From vocational or technical training $\geq 18$ years, up to and including university education. ${ }^{5}$ Participants did not have any current psychotic or substance disorder. ${ }^{a, b, c}$ Indicators of significant simple contrasts. ${ }^{*}$ Groups differ significantly, $p<.05 .{ }^{* * *}$ Groups differ significantly, $p<.005$. 


\section{CHAPTER 2}

types of emotional schema related stimuli and one type of emotional schema unrelated stimuli), Stimulus Valence (negative, positive), and Presentation (supraliminal, subliminal). The dependent variable was emotional Stroop interference, i.e., the S's mean reaction time (RT) on the emotional stimuli of a certain type minus the S's mean RT on the neutral stimuli.

\section{Materials and Apparatus}

\section{Diagnostics}

DSM-IV axis I and II diagnoses were assessed with the Dutch-language versions of the SCID-I (First, Spitzer, Gibbon, \& Williams, 1997; Groenestijn, Akkerhuis, Kupka, Schneider, \& Nolen, 1999) and SCID-II (First, Spitzer, Gibbon, Williams, \& Benjamin, 1994; Weertman, Arntz, \& Kerkhofs, 2000). Current severity of BPD symptoms was measured with the BPD Checklist (range (5235)) (Arntz \& Dreessen, 1992) consisting of 47 5-point Likert scale items on which Ss had to indicate to what degree they are currently troubled by a wide range of DSM-IV BPD symptoms. The BPD Checklist has a very good internal consistency (Cronbach's $\alpha$ BPD Checklist $=.97$ ). For this study, BPD Checklist subscales were created for symptoms of anxiety $(\alpha=.86)$, impulsiveness $(\alpha=.56)$, and identity disturbance $(\alpha=.90)$.

\section{Anxiety measurement}

The Dutch-language version of the State-Trait Anxiety Inventory (STAI) (Spielberger, Gorsuch, \& Lusthene, 1970; van der Ploeg, 1980; Hermans, 1994) measured state and trait anxiety (ranges (20-80)).

\section{Schema assessment}

BPD schemas were assessed with the borderline subscale of the Personality Disorder Belief Questionnaire (PDBQ-120) (Dreessen \& Arntz, 1995). The subscale has a good internal consistency (Cronbach's $\alpha=.87$ ) (Arntz, Dreessen, Schouten, \& Weertman, 2004).

\section{Childhood trauma scores}

Traumas during childhood were assessed with the Structured Childhood Trauma Interview (VBG) (van den Bossche, Kremers, Sieswerda, \& Arntz, 1999). This 
Interview rates occurrences of specific events of serual abuse. physical abuse. physical neglect. emotonal abuse and emotonal neglect and several objective aspects of each event type. Events and their aspects were rated a prion according to degree of damaging potential For example abuse by others more close to the S. at lower age of the S, or with higher frequencies or longer dura. tion, received higher ratings Summing the ratings resulted in three composite childhood trauma scores: sexual (range $10-47$ ). physical (range $(0-76)$. and emotional trauma (range (0-97))

\section{Stroop task}

Cognitive biases were assessed with a computerized emotional Stroop task with one-by-one trials. Each trial began with the presentation of a white cross at the center of a black computer screen for $0.5 \mathrm{~s}$, followed by the stimulus word written in red, blue. green or yellow capitals $0.5 \mathrm{~s}$ after it's disappearance. In subliminal trials, the word was presented during an individually calibrated presentation time ( $8-30 \mathrm{~ms}$ ), after which each letter of the word was replaced by a mask in the same color. Ss had to name as quickly and correctly as possible the color of the stimulus word (supraliminal trials) or masks (subliminal trials), after which the word or masks disappeared. Maximum presentation time of the word was $2 \mathrm{~s}$. The experimenter pressed an error button in case of a wrong response. A next trial started $2.5 \mathrm{~s}$ after appearance of the previous word.

Ten types of words were presented, consisting of 12 different Dutch words each. and matched on mean syllable length ( $1 / \cdots 2.4$, range (1-4)). The word stimuli of the Stroop task belonged to 10 types of words. Six types consisted of BPD schema related negative words and their positive opposites: words related to the schemas 'I am powerless and vulnerable' (e.g., powerless, powerful), 'I am inherently unacceptable' (e.g., unacceptable, worthy), and 'Others are dangerous and malevolent' (e.g., malevolent, reliable). Two types consisted of BPD schema unrelated negative and positive words: words related to stinginess (e.g., stingy), and joyfulness (e.g. joyful). Two final types consisted of neutral words: words related to science (e.g., abstract), and business (e.g., practical).

Specificity of the stimuli was judged by 7 therapists with clinical and theoretical knowledge of BPD related schemas. On the average, $95 \%$ of the negative schema related, $80 \%$ of the positive schema related, $90 \%$ of the negative schema unrelated, $86 \%$ of the positive schema unrelated, and $94 \%$ of the neutral stimuli were correctly classified. Emotional valences of the negative and positive stimuli were judged by 10 non-therapists. The negative stimuli 


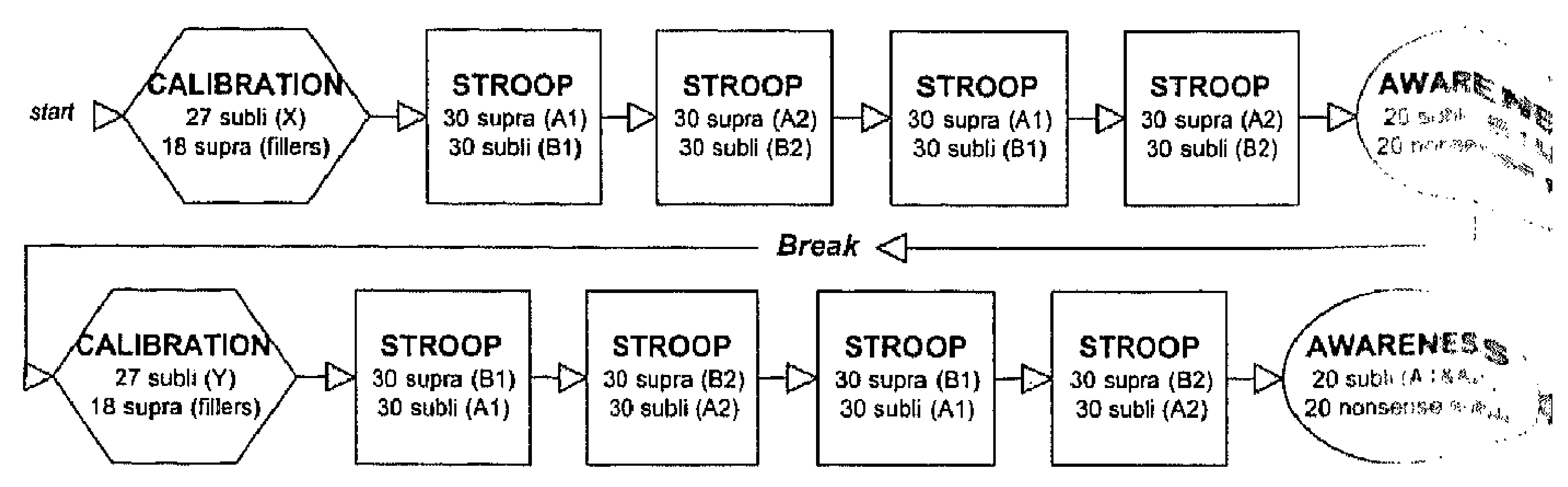

Figure 2.1: Succession of calibration, emotional Stroop, and awareness block numbers of supraliminally and subliminally presented stimuli and indications of $5 \mathrm{ti}$ in sets per block.

were rated more negatively, $t(9)=-10.2, p<.0005$, and the positive sti more positively, $t(9)=7.95, p<.0005$, than the neutral stimuli. Emotional lences of negative schema related and negative unrelated stimuli were sin $t(9)=-1.14, n s$, those of the positive schema related stimuli were less pos that that of the positive schema unrelated stimuli, $t(9)=-4.33, p<.101$. controlled for the latter difference in the analyzes using a restricted set of $>$ । itive schema unrelated stimuli that still matched on mean syllable length, that was equally positive as the schema related stimulus set, $t(9)=1$.fir), Words were not matched on frequency of use because this has been sh not to influence response latencies (Foa \& McNally, 1986; Watts, McKel Sharrock, \& Trezise, 1986; McNally, Riemann, \& Kim, 1990).

For reasons of feasibility, trials were presented in two series of four blo with 60 trials per block. See Figure 2.1 for an illustration of the procedure prevent priming effects within the series, half of the stimulus words were sented supraliminally while the others were presented subliminally. Sublimir presented words in the first series were presented supraliminally in the sec series, and vice versa for the other half of the words. Stimuli were presel two times: one set consisting of half of the words in the first and third. the remaining words in the second and fourth block. These stimulus sets equal mean syllable length. Stimulus sets $x$ order effects were controller by balancing the conditions within and between the groups. Within the blo stimuli appeared in random order and in random color, but with maximally consecutive trials with the same presentation mode (supraliminal, sublimil stimulus type, or color, and with each color used equally frequent. 
We derived interference scores from the RTs of correct responses (98.7\%) that were longer than $300 \mathrm{~ms}$ and shorter than the overall mean RT plus 3 SDs of responses on supraliminal or subliminal stimuli (98.1\%). Sixteen emotional interference scores (one per condition of Stimulus Type $x$ Stimulus Valence $x$ Presentation) resulted from subtracting each S's mean RT on the (supraliminal or subliminal) neutral stimuli from the S's mean RT on the stimuli of one of the eight (supraliminal or subliminal) emotional stimulus types. (RTs on the neutral stimulus types did not differ across Ss, $|t|(65) s<.97, n s$.) Additionally, we calculated two other types of effect scores (one per condition of Stimulus Valence $x$ Presentation) in which four respectively three levels of Stimulus Type were taken together. General emotional interference scores (EMO scores) resulted from averaging the interference scores for all emotional (supraliminal or subliminal, negative or positive) stimuli. Schema specific effect scores (SCHEMA scores) equalled the mean interference score for all schema related stimuli minus the mean interference score for the schema unrelated stimuli.

We trimmed the scores by replacing outliers that fell outside the $95 \%$ confidence interval of the group's mean, by the group's mean plus or minus two SDs. Groups had similar percentages of outliers (BPD: 3\%, CPD: $5 \%$, AID: $6 \%$, NP: $4 \%)$.

\section{Calibration task}

The presentation time of subliminal stimuli was individually assessed with a calibration task. Ss had to read aloud 45 emotional and neutral one-syllable words that were presented one-by-one in 9 series of five stimuli. The first, third and fifth word of each series was presented subliminally and the second and fourth word was presented supraliminally. Presentation times of the subliminal stimuli were $17,12,24,14,27,10,20,8$, and $30 \mathrm{~ms}$ respectively. The longest presentation time at which the subject could not read any of the three subliminally presented words was selected.

\section{Awareness task}

Awareness for subliminally presented stimuli was checked by letting Ss detect whether 40 in random order and subliminally presented stimuli were nonsense words ( 20 trials) or real words ( 20 trials) by pressing one of two buttons. The real words formed a representative subset (two words per word stimulus type, equal number of syllables) of the subliminally presented words in the preceding 


\section{CHAPTER 2}

two Stroop task blocks.

\section{Apparatus}

The tasks were run on a AMD $500 \mathrm{~Hz}$ PC with an Elsa graphical adapter and a 17 inch Eizo F57 monitor. A Stroop monitor interface connected the computer to a microphone, a two-button response box, and an error button. Software programs running on Windows NT4 randomized and presented the stimuli, and recorded response latencies, with an accuracy of $0.5 \mathrm{~ms}$. The room was indirectly and dimly illuminated. Luminous intensity was kept at a predetermined level.

\section{Procedure}

Ss were tested individually. The whole experiment took one to four sessions, depending on the time needed for administration of the SCID interviews. First, the $S$ received general information about the study, signed an informed consent form, and was administered a checklist on demographics and medical information. Then a diagnostic phase followed in which the SCID-I and -II interview, and the BPD Checklist were administered. On a next day or after a break, the S was seated behind the computer screen and continued with calibration task 1 , STAI (trait and state 1), Stroop task 1, awareness task 1, PDBQ-120, and after a break or in a final session, calibration task 2, state part of the STAI (state 2), Stroop task 2, awareness task 2, and the VBG. Having completed the battery, the $S$ was debriefed and received a small financial compensation. The study was approved by the ethical committee of Maastricht University.

\section{Results}

\section{Questionnaires}

Mean scores on the questionnaires are presented in Table 2.2. The BPD group showed higher scores than the control groups. They showed significantly higher scores on the BPD Checklist and the three VBG subscales than all other groups. Their scores on the STAI subscales were significantly higher than those of the non-PD groups. 
Table 2.2: Mean scores on the questionnaires per group ${ }^{1}$.

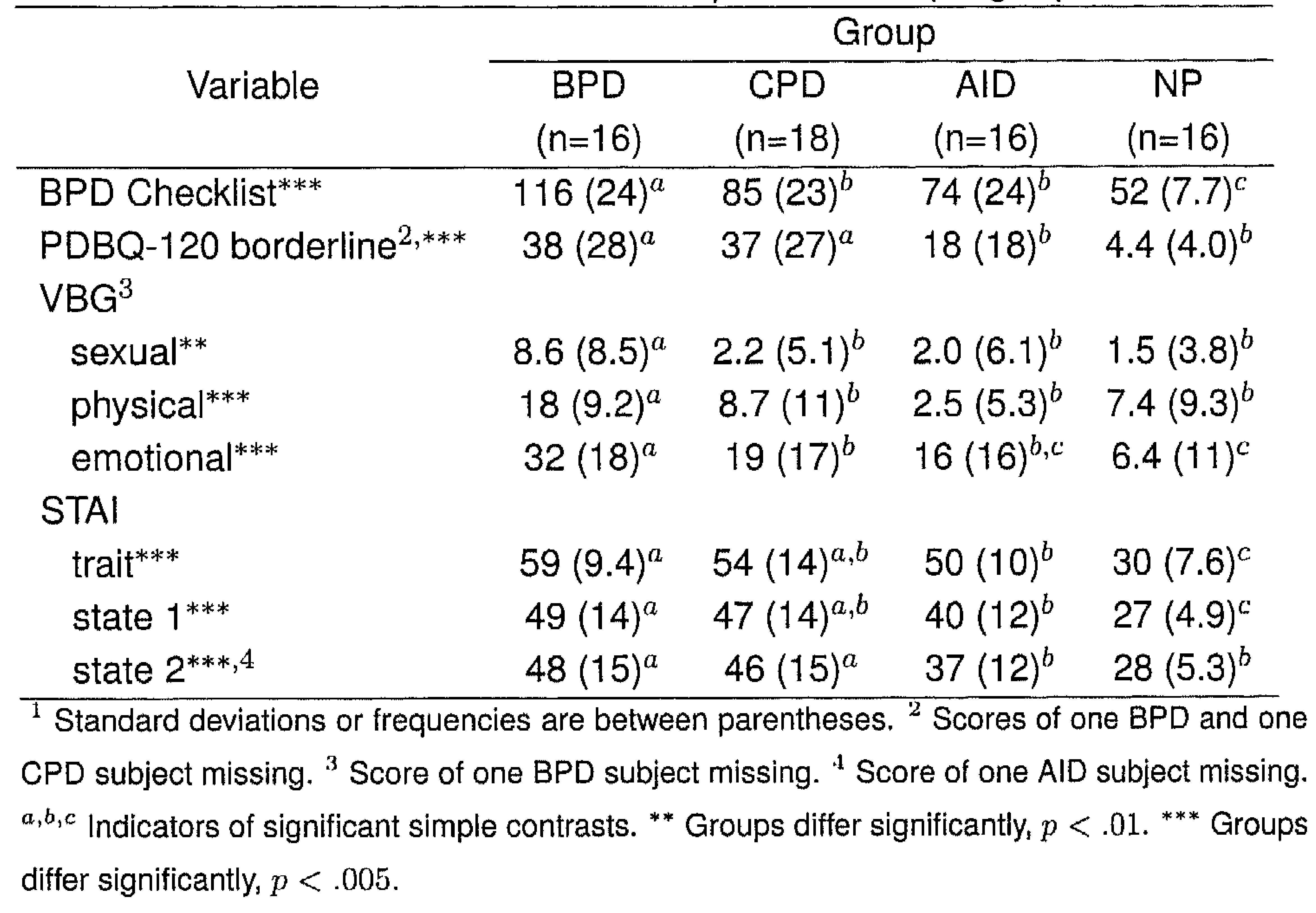

\section{Stroop interference}

\section{Supraliminal stimuli}

Emotional interference scores for supraliminal negative and supraliminal positive stimulus types are shown in Figure 2.2 and Figure 2.3 respectively.

We performed four separate ANOVAs of the EMO and SCHEMA scores for supraliminal negative and positive stimuli. Groups were compared with deviation contrasts (BPD, CPD, AID, and NP vs whole group). Results are presented in Table 2.3.

The ANOVA of the EMO scores for the supraliminal negative stimuli yielded significant contrasts for the BPD group showing higher scores, and the NP group showing lower scores. BPD patients also showed significantly higher SCHEMA scores for the supraliminal negative stimuli than the overall group, whereas the contrasts for the control groups for the SCHEMA scores were not significant or in the opposite direction. Direct tests also showed that BPD patients had higher scores than the whole group (whereas the others had not) for emotional interference scores of schema related stimuli, $t(65)=2.80, p<.005$, one-tailed, but not for emotional interference scores for schema unrelated stim- 


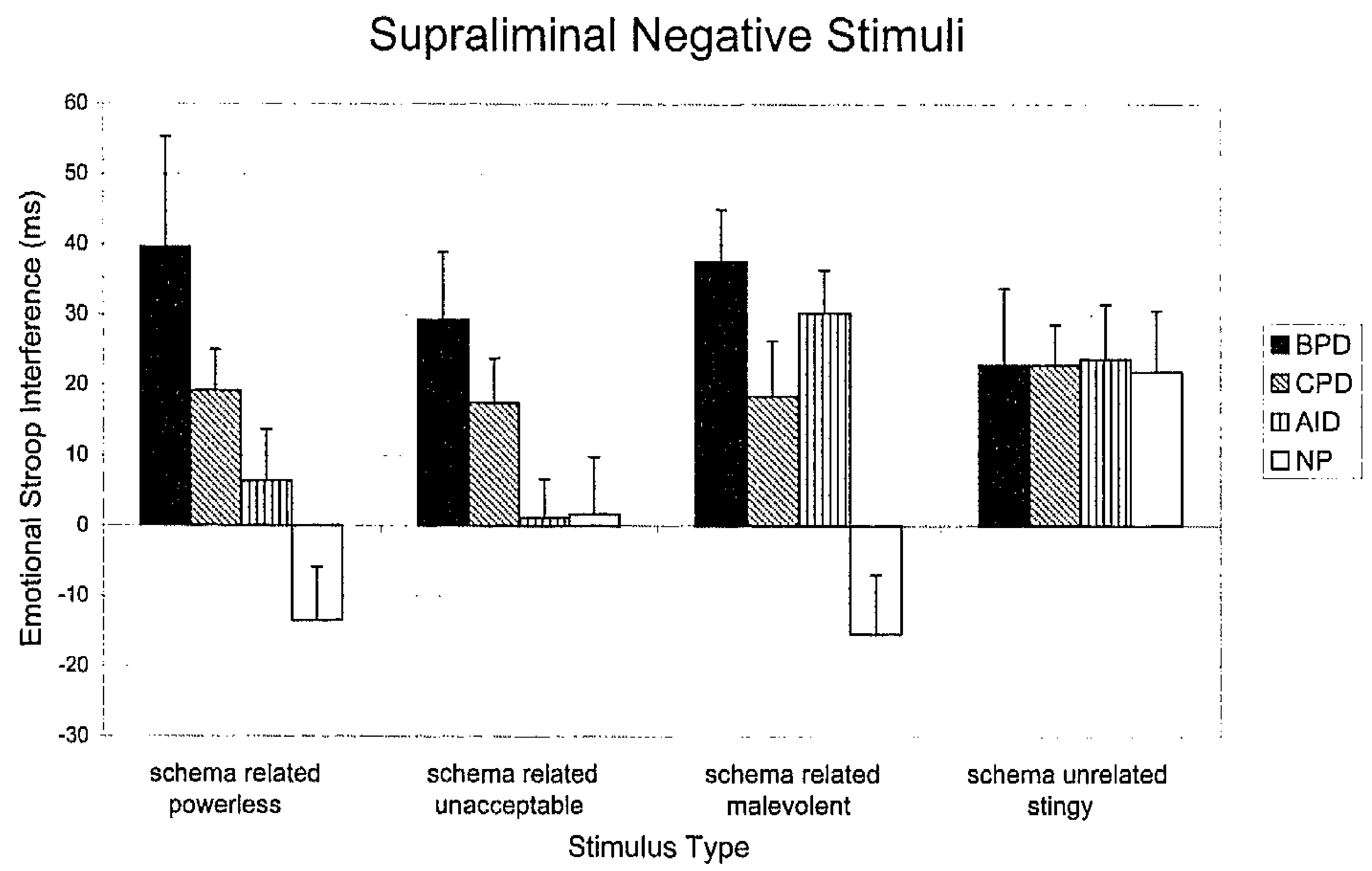

Figure 2.2: Mean emotional interference scores (ms) (+SE) of the BPD $(n=16)$, CPD $(n=18)$, AID ( $n=16)$, and NP ( $n=16)$ group for the supraliminal negative schema related and schema unrelated stimulus types.

uli, $t(65)=.01, n s$. The high EMO scores for the supraliminal negative stimuli of the BPD group, could thus be attributed to the interference of schema related stimuli.

We performed four separate ANOVAs of the EMO and SCHEMA scores for supraliminal negative and positive stimuli. Groups were compared with deviation contrasts (BPD, CPD, AID, and NP vs whole group). Results are presented in Table 2.3.

The ANOVA of the EMO scores for the supraliminal negative stimuli yielded significant contrasts for the BPD group showing higher scores, and the NP group showing lower scores. BPD patients also showed significantly higher SCHEMA scores for the supraliminal negative stimuli than the overall group, whereas the contrasts for the control groups for the SCHEMA scores were not significant or in the opposite direction. Direct tests also showed that BPD patients had higher scores than the whole group (whereas the others had not) for emotional interference scores of schema related stimuli, $t(65)=2.80, p<.005$, one-tailed, but not for emotional interference scores for schema unrelated stimuli, $t(65)=.01, n s$. The high EMO scores for the supraliminal negative stimuli of the BPD group, could thus be attributed to the interference of schema related 


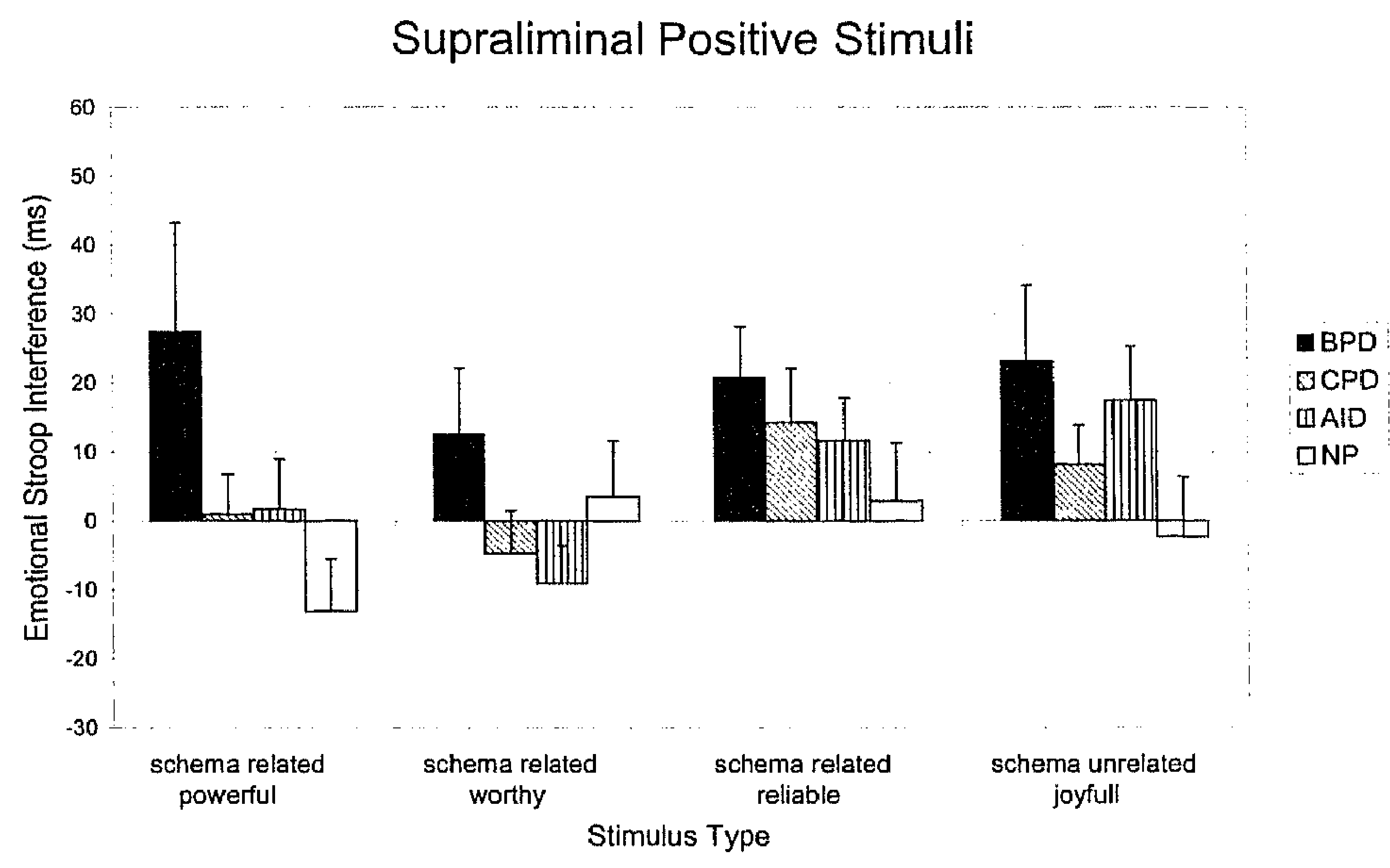

Figure 2.3: Mean emotional interference scores (ms) (+SE) of the BPD ( $n=16)$, CPD $(n=18)$, AID $(n=16)$, and NP ( $n=16)$ group for the supraliminal positive schema related and schema unrelated stimulus types.

stimuli.

In order to examine whether the BPD group showed differential emotional interference scores across the three schema related stimulus types, we performed a repeated measures analysis with Schema Related Stimulus Type (powerless, unacceptable, and malevolent words) as within-subjects factor, BPD Diagnosis (BPD, not BPD) as between-subjects factor, and Emotional Interference score as dependent factor. This analysis showed no effect of Schema Related Stimulus Type, $F(1.75,112)=0.55$, ns, nor an interaction effect of Schema Related Stimulus Type $\times$ BPD Diagnosis, $F(1.75,112)=.58$, ns. Apparently, the BPD group showed similar emotional interference scores for all three schema related stimulus types.

Analyzes for the supraliminal positive stimuli revealed that the four groups had dissimilar EMO scores again, but SCHEMA scores were now similar across the groups. The BPD group stood out with significantly higher EMO scores. A repeated measures analysis of the emotional interference scores with Stimulus Type (powerful, worthy, reliable, and joyful words) and BPD Diagnosis (BPD, not BPD) as factors, showed no effect of Stimulus Type, $F(3,192)=1.64, n s$, nor an interaction effect of Stimulus Type $\times$ BPD Diagnosis, $F(3,192)=1.16$, 
CHAPTER 2

Table 2.3: Deviation contrasts for EMO scores and SCHEMA scores for supraliminal negative and positive stimuli (ms)

\begin{tabular}{ccccc}
\hline Contrast & $M_{\text {Group }}(\mathrm{SD})$ & $M_{\text {ContrastGroup }}(\mathrm{SD})$ & $t$ & $p$ \\
\hline Negative Stimuli & & & & \\
EMO score & & & & \\
BPD vs all & $32.4(40.7)$ & $16.6(29.6)$ & 2.66 & $<.01$ \\
CPD vs all & $19.6(21.7)$ & $16.6(29.6)$ & .53 & $n s$ \\
AID vs all & $15.4(25.6)$ & $16.6(29.6)$ & -.19 & $n s$ \\
NP vs all & $-1.35(18.2)$ & $16.6(29.6)$ & -2.99 & $<.005$ \\
SCHEMA score & & & & \\
BPD vs all & $8.48(50.7)$ & $-9.35(35.9)$ & 2.41 & $<.05$ \\
CPD vs all & $-8.46(28.6)$ & $-9.35(35.9)$ & .13 & $n s$ \\
AID vs all & $-9.25(29.7)$ & $-9.35(35.9)$ & .02 & $n s$ \\
NP vs all & $-28.3(22.2)$ & $-9.35(35.9)$ & -2.55 & $<.05$ \\
Positive Stimuli & & & & \\
EMO score & & & & \\
BPD vs all & $21.0(32.0)$ & $7.14(24.5)$ & 2.27 & $<.01$ \\
CPD vs all & $4.66(17.1)$ & $7.14(24.5)$ & -.52 & $n s$ \\
AID v all & $5.45(18.0)$ & $7.14(24.5)$ & -.35 & $n s$ \\
NP vs all & $-2.25(24.7)$ & $7.14(24.5)$ & -1.78 & $=.07$ \\
SCHEMA score & & & & \\
BPD vs all & $-1.49(46.3)$ & $-4.61(36.3)$ & .41 & $n s$ \\
CPD vs all & $-2.30(27.6)$ & $-4.61(36.3)$ & .32 & $n s$ \\
AID v all & $-18.3(35.4)$ & $-4.61(36.3)$ & -1.75 & $=.09$ \\
NP vs all & $3.39(34.4)$ & $-4.61(36.3)$ & 1.03 & $n s$ \\
\hline
\end{tabular}

$n s$. BPD patients thus showed similar emotional interference scores across all supraliminal positive stimulus types. Analyzes of EMO and SCHEMA scores for the restricted supraliminal positive schema unrelated stimulus set yielded similar effects. We controlled for effects of general state and trait anxiety by running four follow-up ANCOVAs of the EMO scores and SCHEMA scores for supraliminal negative and supraliminal positive stimuli with STAI state 1, state 2 , and trait scores as covariates. This changed the NP group's contrast for EMO scores for supraliminal negative stimuli into a trend, $t(65)=1.91, p=.06$, and this group's contrast for supraliminal positive stimuli into a nonsignificant effect, $t(65)=1,50, n s$. Contrasts for the BPD group remained essentially the same. 
HYPERVIGILANCE IN BPD: SPECIFICITY, AUTOMATICITY, AND PREDICTORS

\section{Subliminal stimuli}

Presentation times selected by the calibration tasks were similar across the groups, Kruskall-Wallis $\chi^{2} s(3, N=66)<1.93$, ns.

Mean hit rate in awareness task $1(M=.52, S D=.068$, range (.36-.73)) was higher than $.50, t(65)=2.12, p<0.05$, one-tailed. Mean hit rate in awareness task $2(M=.50, S D=.11$, range (.29-.81)) was not higher than $.50, t(65)=-.13, n s$, one-tailed. Six Ss in task 1 and one $S$ in task 2 showed hit rates close to mean hit rate plus $3 \mathrm{SD}$. After having removed their responses in these tasks, mean hit rate in task $1(M=.51, S D=.063$, range (.36-.60)) and task $2(M=.49, S D=.10$, range (.29-.75)) were both not higher than .50 , $|t| s<.72, n s$, one-tailed. RTs on subliminal stimuli of Stroop task 1 of the six Ss whose hit rates were too high in awareness task 1 , and RTs on subliminal stimuli Stroop task 2 of the $S$ whose hit rate was too high in awareness task 2, were removed from the analyzes. Interference and effect scores for the subliminal stimuli of these Ss were calculated with their remaining RTs.

EMO and SCHEMA scores for the subliminal negative and positive stimuli were also analyzed with four ANOVAs. Table 2.4 summarizes the results. Analyzes only revealed a significant contrast for the NP group showing lower SCHEMA scores for subliminal negative stimuli. Contrasts for the BPD and AID patients' SCHEMA scores for the subliminal negative stimuli were in the other direction, but did both not reach significance. Analyzes of EMO and SCHEMA scores for the restricted set of subliminal positive schema unrelated stimuli yielded similar effects. Adding STAI state 1, state 2, and trait scores weakened the AID group's contrast, $t(65)=1.69, p=.10$, and removed the effects for the NP and BPD group.

\section{Predictors and comorbidity}

In order to detect potential predictors of cognitive bias, as well as to disentangle relations between comorbid diagnoses and bias, we performed 9 multiple regression analyzes on the whole sample $(\mathrm{N}=66)$. Independent factors in these analyzes were diagnosis (fulfilling DSM-IV criteria for BPD, a CPD, an anxiety disorder, or a mood disorder), BPD symptom cluster (anxiety, impulsiveness, identity disturbance), or childhood trauma (sexual, physical, emotional). The dependent variables were those scores on which the BPD patients stood out in the previous analyzes, i.e., the SCHEMA score for the supraliminal negative stimuli, the EMO score for the supraliminal positive stimuli, and the SCHEMA 


\section{CHAPTER 2}

Table 2.4: Deviation contrasts for EMO scores and SCHEMA scores for subliminal stimuli (ms).

\begin{tabular}{ccccc}
\hline Contrast & $M_{\text {Group }}(\mathrm{SD})$ & $M_{\text {ContrastGroup }}(\mathrm{SD})$ & $\mathrm{t}$ & $\mathrm{p}$ \\
\hline Negative Stimuli & & & & \\
EMO score & & & & \\
BPD vs all & $0.08(37.2)$ & $4.28(23.6)$ & -.67 & $n s$ \\
CPD vs all & $7.64(16.9)$ & $4.28(23.6)$ & .72 & $n s$ \\
AID vs all & $-4.17(14.4)$ & $4.28(23.6)$ & -1.67 & $n s$ \\
NP vs all & $12.4(17.8)$ & $4.28(23.6)$ & 1.64 & $n s$ \\
SCHEMA score & & & & \\
BPD vs all & $16.9(27.9)$ & $5.08(30.1)$ & 1.87 & $=.06$ \\
CPD vs all & $-3.87(25.1)$ & $5.08(30.1)$ & -1.57 & $n s$ \\
AID vs all & $17.1(27.5)$ & $5.08(30.1)$ & 1.92 & $=.06$ \\
NP vs all & $-8.72(33.0)$ & $5.08(30.1)$ & -2.30 & $<.05$ \\
Positive Stimuli & & & & \\
EMO score & & & & \\
BPD vs all & $-5.29(28.1)$ & $1.31(21.8)$ & -1.39 & $n s$ \\
CPD vs all & $6.14(16.1)$ & $1.31(21.8)$ & 1.12 & $n s$ \\
AID vs all & $-3.29(18.1)$ & $1.31(21.8)$ & -.96 & $n s$ \\
NP vs all & $7.07(22.6)$ & $1.31(21.8)$ & 1.27 & $n s$ \\
SCHEMA score & & & & \\
BPD v all & $-6.43(37.3)$ & $1.89(31.6)$ & -1.20 & $n s$ \\
CPD vs all & $4.09(34.4)$ & $1.89(31.6)$ & .34 & $n s$ \\
AID vs all & $3.43(27.8)$ & $1.89(31.6)$ & .23 & $n s$ \\
NP vs all & $6.18(26.8)$ & $1.89(31.6)$ & .63 & $n s$ \\
\hline
\end{tabular}

score for the subliminal negative stimuli. Results of these analyzes are summarized in Table 2.5. In order to control for an artificial enhancement by including a non-clinical sample, we controlled whether results were similar when only patients were included. This appeared to be the case, except for a clearly weaker relation $(\beta=.15, p=.32)$ between the diagnosis anxiety disorder and the SCHEMA score for the subliminal negative stimuli.

BPD diagnosis was the only diagnosis that showed significant relations with the effect scores for supraliminal schema related negative stimuli and supraliminal schema related and unrelated positive stimuli. Effect scores for subliminal schema related negative stimuli showed similar borderline significant or significant $\beta$ s for the diagnoses BPD and anxiety disorder respectively. The latter 
HYPERVIGILANCE IN BPD: SPECIFICITY, AUTOMATICITY, AND PREDICTORS

Table 2.5: Summary of nine regression analyzes for variables predicting SCHEMA scores for supraliminal negative stimuli, EMO scores for supraliminal positive stimuli, or SCHEMA scores for subliminal negative stimuli $(\mathrm{N}=66)$.

\begin{tabular}{|c|c|c|c|c|c|c|}
\hline \multirow[b]{3}{*}{ Independent Variable } & \multicolumn{6}{|c|}{ Dependent Variable } \\
\hline & \multicolumn{2}{|c|}{$\begin{array}{l}\text { SCHEMA score } \\
\text { Supra. Negative }\end{array}$} & \multicolumn{2}{|c|}{$\begin{array}{c}\text { EMO score } \\
\text { Supra. Positive }\end{array}$} & \multicolumn{2}{|c|}{$\begin{array}{l}\text { SCHEMA score } \\
\text { Sub. Negative }\end{array}$} \\
\hline & $\bar{\beta}$ & $p$ & $\bar{\beta}$ & $p$ & $\beta$ & $p$ \\
\hline \multicolumn{7}{|l|}{ Diagnosis } \\
\hline BPD & .28 & $<.05$ & .32 & $<.05$ & .24 & $=.05$ \\
\hline CPD & .25 & $=.08$ & .066 & $n s$ & -.11 & $n s$ \\
\hline Anxiety disorder & .061 & $n s$ & -.049 & $n s$ & .28 & $<.05$ \\
\hline Mood disorder & -.10 & $n s$ & -.003 & $n s$ & -.14 & $n s$ \\
\hline \multicolumn{7}{|l|}{ BPD symptom clusters ${ }^{1}$} \\
\hline anxiety & .44 & $<.05$ & -.38 & $=.08$ & .02 & $n s$ \\
\hline impulsiveness & -.16 & $n s$ & .086 & $n s$ & .04 & $n s$ \\
\hline identity disturbance & .016 & $n s$ & .59 & $<.01$ & .10 & $n s$ \\
\hline \multicolumn{7}{|l|}{ Childhood trauma ${ }^{2}$} \\
\hline sexual & .38 & $<.005$ & .19 & $n s$ & -.09 & $n s$ \\
\hline physical & .25 & $=.08$ & -.02 & $n s$ & -.04 & $n s$ \\
\hline emotional & -.14 & $n s$ & 23 & $n s$ & .21 & $n s$ \\
\hline
\end{tabular}

Note. SCHEMA score supraliminal negative stimuli: $R^{2}=.14, n s$ for Diagnosis; $R^{2}=.19$, $p<.001$ for Childhood trauma; $R^{2}=.15 ; p<.05$ for BPD symptom clusters. EMO score supraliminal positive stimuli: $R^{2}=.11, n s$ for Diagnosis; $R^{2}=.10, n s$ for Childhood trauma; $R^{2}=.15, p<.05$ for BPD symptom clusters. SPE score subliminal negative stimuli: $R^{2}=.14$, $p=.06$ for Diagnosis; $R^{2}=.04, n s$ for Childhood trauma; $R^{2}=.02 ; n s$ for BPD symptom clusters. ${ }^{1}$ BPD Checklist subscale scores. ${ }^{2}$ VBG subscale scores.

however was now longer significant in an analysis without the non-clinical group and might thus be artificial.

BPD severity, especially the severity of BPD anxiety symptoms (such as separation anxiety), showed a significant relation with the SCHEMA score for supraliminal negative stimuli. Severity of BPD impulsiveness and identity disturbance symptoms were not significantly related to the SCHEMA score for supraliminal negative stimuli. The EMO score for the supraliminal positive stimuli on the other hand was significantly and positively related to severity of BPD identity disturbance, and tended to be negatively instead of positively related to severity of BPD anxiety symptoms.

Having experienced (more severe) childhood sexual or physical traumas showed significant or nearly significant $\beta$ s with the SCHEMA score for nega- 
CAAPTED?

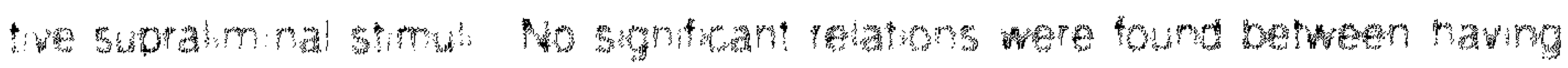

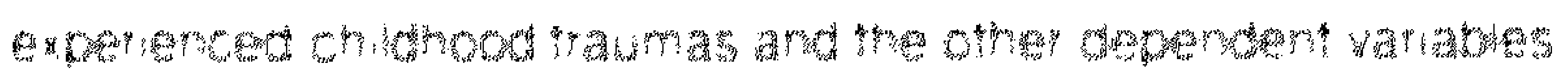

\section{Discussion}

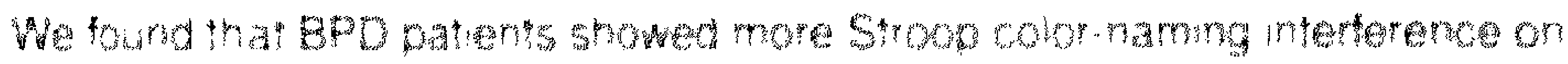

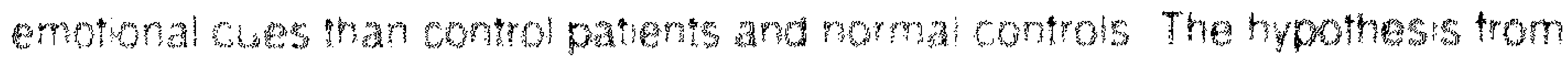
the cognt:

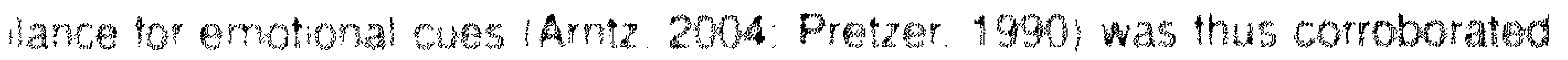

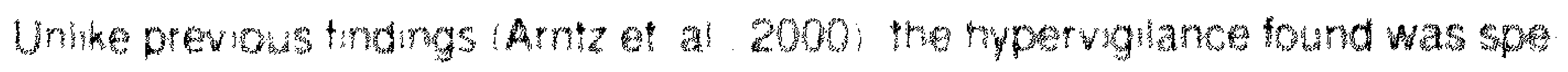
chtc tor $\mathrm{BPD}$ patents and was not fourd in chuster $\mathrm{CPO}$. nor in axis 1 patents The typernglance appeared furthermore spectically pelated to BPD. and not to other comortud disorders of the patuents

BPD patents showed hypervglance for negatre as well as positive strmum. but our results indicate that BPD patents do not show a general form of hy. perviglance. Which one may infer from a general defout m emotional regulation Firstly, BPD patients showed a bias for schema related negatwe cues, but not for schema unrelated negatwe cues H.e. person descriptors related to sting. ness). Secondy, bases for negative and positve strmuli shown by the BPD group of this study seerned to differ conceptuaty Hypervigiance for schema related negative stmul was related to current anxiety (e.g. BPD anxiety symp. toms; and predicted by a history of chilhood sexual abuse whereas hyper. vigiance for positive stmuli was not (positively) related to current anxiety and not predicted by chidhood sexual abuse or other childnood traumas The first type of hypervgitance might be rooted in (childhood) traumas, conform theo. res viewing BPD as a complex post traumatic anxiety disorder (Herman et al. 1989. Sabo. 1997, Zanarm. 1997). Together these findings support the hypoth. esis that BPD patients suffer from cognitive schema mediated hypervigilance for specific negative cues as formulated in the cognitive model of BPD (Arrtz. 2004; Pretzer. 1990)

It is not clear how to understand the bias for positive stimuli in BPD which was neither related to stronger current anxiety nor predcted by childhood trauma. This bias might represent an urge to approach a certain positive object, for ex. ample a strong and caring figure. Such an 'urge to act' was also hypothesized for appetitive biases for alcohol or food cues found in tasting and alcoholic persons iLavy \& van den Hout, 1993, Johnsen, Laberg, Cox, Vaksdal, \& Hugdaht 1994). Another explanation taking into account the positive relation found be 
veen bias for positive stimuli and BPD identity disturbance and the fact that o specificity was found here, is that the combining of positive stimuli and negtive schemas may result in an ambiguous situation that has been thought to e arousing for BPD patients, especially for those patients with a weak identity 2retzer, 1990). A similar phenomenon might have caused amygdala activation fter the presentation of neutral faces in patients with BPD (Donegan et al., 003).

The question whether hypervigilance in BPD is also mediated by schemas perating on cues below awareness, could not yet be confirmed with certainty. Iso on an automatic level, BPD patients attended more to schema related than chema unrelated negative cues, although now not significantly more $(p=.06)$. 'atients with an axis I disorder, most of whom (94\%) having an anxiety disorder, terestingly showed a similar trend. This might again indicate that BPD and nxiety patients share basic characteristics. It can be speculated that both atient groups monitor the presence of BPD schema related stimuli, which in zct are basic social threat cues, at a nonconscious level, but only patients ithout BPD manage to strategically suppress this urge at a conscious level, a ypothesis that also has been put forward to explain a similar pattern found for linical and non-clinical high trait anxious subjects (Williams et al., 1997).

This study had several strengths. It included two patient control groups vhich ruled out effects of axis I and other axis II disorders. Inclusion of two con- ol emotional stimulus types (schema unrelated stimuli and emotionally positive timuli) controlled for schema related and negative emotional effects. Presenation time was further assessed individually which optimized detection of subminal biases.

Future studies could include larger samples to lower chance of type ll errors, ,articularly concerning conclusions on the small subliminal effects. A longitudiral design could rule out uncontrolled between-subject factors, and an expermental design in which hypervigilance is manipulated might clarify the causal ;tatus of hypervigilance in BPD. Alternative paradigms could also be considered secause emotional Stroop bias may result from later cognitive processes than ittention (e.g., response), or related to only the quality of the schemas (e.g., powerless', 'malevolent') but not to the specific qualified object (e.g., 'poweress self' or 'malevolent other').

For clinical practice, more attention to hypervigilance in BPD is recomnended. This study empirically supports that hypervigilance for emotional, sspecially schema related negative cues, is specific for BPD, a characteristic 


\section{CHAPTER 2}

Which has a maintaining or causal status in the cognitive model of this PD. Although BPD patients might still benefit from general emotion regulation training, this study suggests that a focus on (1) correction of BPD schemas, for example by cognitive and experiential techniques. and (2) reduction of vigilance for negative cues related to BPD schemas or for generally positive person related cues, for example by (cue-)exposure techniques and attentional training (Arntz. 2004: Pretzer, 1990; Young, Klosko. \& Weishaar, 2003: Layden et al., 1993). might be preferable. 


\title{
Chapter 3
}

\section{Successful Psychotherapy Reduces Hypervigilance in Borderline Personality Disorder ${ }^{1}$}

\begin{abstract}
Several studies have demonstrated that patients with borderline personality disorder (BPD) show hypervigilance or selective attention to emotional cues, which is in line with cognitive models of BPD. The present study aimed to further investigate selective attention in BPD, in particular whether it is content-specific and influenced by treatment. We compared emotional Stroop interferences of stimulus types that were related and unrelated to hypothesized BPD schemas, (1) of patients with BPD $(n=24)$ and nonpatient controls $(n=23)$, and (2) of BPD patients $(n=16)$ at start and end of an intensive, three year lasting treatment. Patients with BPD showed general hypervigilance, i.e., attentional biases for both schema related and unrelated emotional stimuli. Interference by stimuli associated with being generally bad seemed to be most characteristic of BPD. Hypervigilance was completely reduced to normalized levels in recovered patients $(n=6)$, but not in non-recovered patients $(n=10)$ at the end of treatment. Findings support the possibility of structural change in BPD.
\end{abstract}

\footnotetext{
${ }^{1}$ The content of this chapter has been published as Sieswerda, S., Arntz, A., \& Kindt, M. (2007), Successful psychotherapy reduces hypervigilance in borderline personality disorder, Behavioural and Cognitive Psychotherapy, 35, 387-402.
} 


\section{CHAPTER 3}

\section{Introduction}

According to cognitive views, patients with borderline personality disorder (BPD) show dysfunctional schemas and information processing biases resulting in anxiety and hypervigilance. In particular, Pretzer (1990) describes three schemas (core beliefs) that seem to be central in BPD, i.e., 'I am powerless and vulnerable', 'I am inherently unacceptable', and 'The world is dangerous and malevolent'. Regarding the world as dangerous and themselves as relatively powerless and unacceptable, borderline patients can be assumed to feel like a child left alone in a dangerous place (see also, Arntz, 1994). Several empirical findings are in line with this anxious side of BPD. Comorbidity studies of patients with BPD demonstrate that anxiety disorders and anxious cluster personality disorders (PDs) are highly prevalent in BPD (Zanarini et al., 1998b, 1998c). Furthermore, many patients with BPD report histories of childhood traumas (e.g., Herman et al., 1989; Zanarini et al., 2000).

Cognitive theory places anxiety and related biases for threat primarily in an early information processing stage. In this stage, information enters the cognitive system and anxious individuals selectively focus their attention on the origins of threat (e.g., MacLeod, 1991). Attending to stimuli that are real threats has survival value. However, attending to stimuli that are not really threatening, is not adaptive. The latter is thought to lead anxious individuals into a vicious circle of more attention and more anxiety. Selective attention has been found to characterize anxiety disorders (see, Williams et al., 1997), to predict their emergence (MacLeod \& Hagan, 1992; van den Hout et al., 1995), and to cause dysfunctional anxiety (MacLeod, Rutherford, Campbell, Ebsworthy, \& Holker, 2002).

Previous studies on selective attention in BPD (chapter 2 of this thesis; Arntz et al., 2000; Waller \& Button, 1999) consistently found evidence for attentional biases in BPD. These studies, successfully applied an emotional Stroop task, and found that the responses of BPD patients to emotional words were slower than to neutral words. Moreover, this response latency for emotional words in BPD patients was larger than in nonpatients. These findings are in line with a neurobiological study on BPD finding enhanced activation in the amygdala and the fusiform gyrus in the perceptual cortex, associated with anxiety and increased attention for emotionally relevant stimuli respectively (Herpertz et al., 2001a).

It is currently unclear whether BPD patients selectively attend to content- 
specific stimuli or whether they show general hypervigilance to any emotional cue. Two studies (chapter 2 of this thesis; Waller \& Button, 1999) found selective attention for certain stimulus types in BPD, which was not found in normal, and axis I or axis II patient controls. We demonstrated a bias specifically for negative stimuli associated with the three core beliefs formulated by Pretzer (1990) (e.g., powerless, unacceptable, malevolent) and not for control negative emotional stimuli that were not associated with these core beliefs (e.g., stingy). Waller and Button (1999) found a bias for stimuli related to self-criticism (e.g., failure, stupid), and not for stimuli related to physical threat, nor for stimuli related to other-criticism (e.g., ridiculed, humiliated). Arntz et al. (2000) could not demonstrate a specific attentional bias in BPD, and found that these patients as well as Cluster C PD patients attended to negative emotional stimuli in general. The issue of content-specificity is important for several reasons. A specific bias conflicts with the popular hypothesis that patients with BPD show a general, possibly congenital, hyper-emotionality (Linehan, 1993). Finding a specific attentional bias would further differentiate BPD from other disorders and provide more focus to treatments for BPD.

Another important but uninvestigated question is whether selective attention in BPD patients has decreased after successful treatment. A decreased bias in a longitudinal design would more convincingly demonstrate that selective attention is characteristic for BPD than cross-sectional studies, because this design rules out confounding effects of uncontrolled between-subject differences. Moreover, a decreased bias in recovered BPD patients hints at a fundamental change by treatment. Selective attention for emotional stimuli, as measured by an emotional Stroop task, has been found to correlate with functioning on fundamental levels. FMRI studies with healthy volunteers (Whalen et al., 1998) and patients with PTSD have found that emotional Stroop tasks in particular recruit the rostral anterior cingulate cortex (Shin et al., 2001), a part of the brain that has been suggested to be involved in regulatory responses to emotional stimuli. It therefore seems justifiable to conclude that finding a decrease in selective attention in BPD patients who are successfully treated would support the hypothesis that structural changes in BPD are possible, and would do so more strongly than a demonstration of a decrease in self-report measures alone. Such a finding would also support the attainability of fundamental change that is aimed for in new treatments for BPD, for example Beckian therapies (Pretzer, 1990; Layden et al., 1993; Arntz, 1994) and Schema Focused Therapy (Young et al., 2003; Arntz, 2004), which aim to replace maladaptive 


\section{CHAPTER 3}

basic assumptions or schema modes by healthier alternatives, and psychodynamic treatments like Transference Focused Psychotherapy (Clarkin, Yeomans, \& Kernberg, 1999), which target integration of split internalized object relations.

Selective attention has been shown to decrease after successful treatment in several other disorders, including social phobia (Lundh \& Öst, 2001; Mattia, Heimberg, \& Hope, 1993), spider phobia (Côté \& Bouchard, 2005; Lavy \& van den Hout, 1993; Lavy, van den Hout, \& Arntz, 1993; van den Hout, Tenney, Huygens, \& de Jong, 1997; Watts et al., 1986), obsessive-compulsive disorder (Foa \& McNally, 1986), general anxiety disorder (Mathews, Mogg, Kenthish, \& Eysenck, 1995; Mogg, Bradley, Millar, \& White, 1995), anorexia nervosa (Ball, 1999), bulimia nervosa (Cooper \& Fairburn, 1994), major depressive disorder (Segal \& Gemar, 1997), and somatoform disorders (Lupke \& Ehlert, 1998). However, decrease of selective attention does not always coincide with decrease of symptoms (Carter, Bulik, Mclntosh, \& Joyce, 2000; Devineni, Blanchard, Hickling, \& Buckley, 2004; Kampman, Keijsers, Verbraak, Näring, \& Hoogduin, 2002). Although some authors therefore conclude that selective attention is not a useful measure for therapy evaluation, an alternative explanation for the absence of a bias decrease in some studies might be a lack of structural changes, presumably leaving the patient at a relatively high risk for relapse.

In sum, the present study was designed to test the following hypotheses. First, BPD is characterized by a content-specific attentional bias as opposed to a bias for emotional stimuli in general. Second, successful treatment of BPD results in a concomitant decrease of (content-specific or general) bias. The hypotheses were tested for both supra- and subliminal stimuli because it has been assumed that threat related stimuli are attended to automatically and independently of awareness (Beck, 1976).

\section{Method}

\section{Sample}

The experimental group consisted of 24 outpatients with BPD from a Community Mental Health Center starting treatment for BPD. Sixteen of these patients completed the treatment and were tested at end of treatment. At that time, six of them scored below a dysfunctional cutoff score of 15 on the Borderline Personality Disorder Severity Index (BPDSI: Giesen-Bloo, Wachters, Schouten, \& 
Arntz, 2006c) and were regarded as recovered. Nonpatient controls (NPs) were 23 persons without psychopathology who were recruited by advertisements.

Participants were screened with Dutch versions of the SCID-I (First et al., 1997; Groenestijn et al., 1999) and SCID-II interviews (Spitzer, First, Gibbon, \& Williams, 1995; Weertman et al., 2000) for DSM-IV (APA, 1994) axis I and II disorders, the BPD Symptom Checklist 47 (BPD-47: Arntz \& Dreessen, 1992) or the BPDSI (Giesen-Bloo et al., 2006c). Participants that were thought to have dissociative disorders were additionally screened with the SCID-D (Steinberg, 1993). Patients in the BPD group had BPD according to DSM-IV criteria as main diagnosis and a BPDSI score $\geq 20$. They were not allowed to have a (current) psycho-organic disorder, attention-deficit/hyperactivity disorder, severe addiction needing clinical detoxification, bipolar disorder, psychotic disorder, dissociative identity disorder, or antisocial personality disorder. NPs were not allowed to have any current axis I or II disorder, to meet any of the DSMIV BPD criteria, a BPD-47 score $\geq 80$, and more than one axis I disorder in complete remission. General exclusion criteria were age $<18$ and $>60$ years, intoxication by alcohol or drugs during testing, IQ below 80 , vision problems, and not being native speaker of Dutch. The control group was matched to the BPD group on age and sex.

Table 3.1 presents the demographic variables and diagnostic characteristics at screening for the BPD group that started treatment, the NPs, and the subgroup of BPD patients that were assessed at start and end of treatment. The BPD and NP group differed in terms of educational level such that the patients were somewhat less educated than the normal controls; the groups were furthermore quite similar on demographic variables. Axis I disorders that were most frequently diagnosed among the BPD patients were anxiety and mood disorders. Almost half of the BPD patients had an additional cluster C PD and about a quarter had a comorbid cluster A PD.

\section{Design}

The study consisted of two sub-studies both with mixed between-within subjects designs. Independent variables of the first study were: Group (BPD, NP), Stimulus Type (three types of emotional schema related stimuli and two types of emotional schema unrelated stimuli), and Presentation (supraliminal, subliminal). The second study compared recovered to non-recovered BPD patients (Group), at start and end of treatment (Time), the same five types of stimuli 
CHAPTER 3

Table 3.1: Demographic and diagnostic variables at screening for the BPD group, the NP group, and the sub-group of patients with BPD that were tested at start and end of treatment $^{1}$

\begin{tabular}{|c|c|c|c|}
\hline \multirow[b]{2}{*}{ Variable } & \multicolumn{3}{|c|}{ Group } \\
\hline & $\begin{array}{c}\text { BPD } \\
(n=24)\end{array}$ & $\begin{array}{c}N P \\
(n=23)\end{array}$ & $\begin{array}{l}\text { BPD Sub-group } \\
\qquad(n=16)\end{array}$ \\
\hline M Age & $29.6(7.2)$ & $34(11)$ & $28.8(6.6)$ \\
\hline$\%$ Female & $88(21)$ & $91(21)$ & $81(13)$ \\
\hline$\%$ Low - middle level education ${ }^{2}$ & $71(19)$ & $57(13)$ & $75(12)$ \\
\hline$\%$ Employed or student ${ }^{3}$ & $75(18)$ & $78(18)$ & $82(13)$ \\
\hline$\%$ Married/cohabiting & $42(10)$ & $48(11)$ & $44(7)$ \\
\hline \multicolumn{4}{|l|}{ SCID-1 $\left.\right|^{4}$} \\
\hline$\%$ substance & $42(10)$ & $0(-)$ & $44(7)$ \\
\hline$\% \operatorname{mood}$ & $71(17)$ & $0(-)$ & $81(13)$ \\
\hline$\%$ anxiety & $96(23)$ & $0(-)$ & $94(15)$ \\
\hline$\%$ dissociation & $25(6)$ & $0(-)$ & $31(5)$ \\
\hline$\%$ somatoform & $29(7)$ & $0(-)$ & $31(5)$ \\
\hline$\%$ eating & $25(6)$ & $0(-)$ & $13(2)$ \\
\hline M \# current disorders & $3.9(1.2)$ & $0(-)$ & $3.8(1.3)$ \\
\hline \multicolumn{4}{|l|}{ SCID-II } \\
\hline$\%$ cluster $\mathrm{A}$ & $25(6)$ & $0(-)$ & $25(4)$ \\
\hline$\%$ cluster B & $100(24)$ & $0(-)$ & $100(16)$ \\
\hline$\%$ cluster C & $42(10)$ & $0(-)$ & $44(7)$ \\
\hline M\# disorders & $2.1(1.4)$ & $0(-)$ & $2.2(1.4)$ \\
\hline
\end{tabular}

${ }^{1}$ Standard deviations or frequencies are between parentheses. ${ }^{2}$ From no education up to and including vocational or technical training $<18$ years; other participants had vocational or technical training $\geq 18$ years, up to and including university education. ${ }^{3}$ Other participants were unemployed and not student. ${ }^{4}$ Participants did not have any current psychotic disorder. 
(Stimulus Type), presented supra- or subliminally (Presentation). The dependent variable in both studies was Emotional Stroop Interference (ESI) score, i.e. the participant's mean reaction time (RT) on the emotional stimuli of a certain type minus the participant's mean RT on the neutral stimuli.

\section{Psychological treatment}

The patients participated in a randomized clinical trial (Giesen-Bloo et al., 2006b) that compared Schema Focused Therapy (Young et al., 2003), a cognitive behavioral treatment with experiential elements, to Transference Focused Psychotherapy (Clarkin et al., 1999), a psychodynamic treatment based on Kernberg's model of borderline personality organization. Both treatments aim at structural change in BPD. Treatments lasted 3 years with a frequency of two sessions a week, and were given by experienced psychotherapists receiving supervision by experts (viz. Jeffrey Young or Frank Yeomans) and participating in frequent peer supervision.

\section{Material}

\section{Diagnostics}

Dutch-language versions of the SCID-I (First et al., 1997; Groenestijn et al., 1999), SCID-D (Steinberg, 1993), and SCID-II (First et al., 1994; Weertman et al., 2000) were used to assess DSM-IV diagnoses. The SCID-II has a good test-retest interrater reliability (Weertman, Arntz, Dreessen, Velzen, \& Vertommen, 2003). Current severity of BPD symptoms was measured with the BPDSI (Giesen-Bloo et al., 2006c) and the BPD-47 (Arntz \& Dreessen, 1992). The BPDSI is a semi-structured interview assessing frequency and severity of DSMIV BPD symptom manifestations during the past three months (range: 0-90). It has an excellent interrater reliability $(0.98 \leq \mathrm{ICCs} \leq 1.00)$ and internal consistency (Cronbach's $\alpha=.96$ ), and a good discriminant ( $p s<.001$ ) and concurrent validity $(.68 \leq r s \leq .77)$. It's dysfunctional cutoff score is 15 and has high specificity and sensitivity (Giesen-Bloo et al., 2006c). The BPD Checklist is a self-report questionnaire consisting of 475 -point Likert scale items on which participants can indicate to what degree they have been troubled by a wide range of DSM-IV BPD symptoms during the past month (range: 47-235). The BPD Checklist has a very good internal consistency (Cronbach's $\alpha>.92$ ), construct, concurrent, and discriminant validity, and has been proved sensitive to 


\section{CHAPTER 3}

change during treatment (Arntz et al, 2003; Giesen-Bloo, Arntz, \& Schouten, 2006a).

\section{Anxiety measurement}

State and trait anxiety were assessed with the Dutch-language version of the State-Trait Anxiety Inventory (STAl: Spielberger et al. 1970; van der Ploeg. 1980) (subscale ranges: 20.80 ). This is a reliable and valid questionnaire (Hermans. 1994). Participants have to report on two scales, each consisting of 20 4.point Likert items, to what degree they feel anxious or tense at the moment or in general.

\section{Emotional Stroop task}

Cognitive biases for schema related and unrelated emotional stimuli were assessed with a computerized emotional Stroop task with one-by-one trials. Although the emotional Stroop task has been criticized in the past for not controlling for response bias (e.g. Dalgleish \& Watts, 1990), more recent findings show that Stroop-like interferences are not caused by response bias (e.g. Luo, 1999). A particular advantage of using this task was the possibility to related current outcomes to earlier emotional Stroop findings.

Each trial of the Stroop task began with the presentation of a white cross at the center of a black computer screen for $.5 \mathrm{~s}$. This was followed by the stimulus word written in red, blue, green or yellow capitals after $0.5 \mathrm{~s}$. Subliminally presented words stayed on the screen for $14 \mathrm{~ms}$, after which the letters of the word were replaced by an array of masks in the same color as the word. Participants were asked to name as quickly as possible the color of the stimulus word (supraliminal presentation) or the post-stimulus masks (subliminal presentation). The experimenter pressed an error button in case of a wrong voice response. The word or mask was presented until the $S$ responded, with a maximum presentation time of $2 \mathrm{~s}$. The next trial started $2.5 \mathrm{~s}$ after the previous stimulus word had appeared.

Seven types of Dutch word stimuli were presented in the Stroop task: negative words related to the BPD schemas on powerlessness (e.g., powerless, vulnerable, helpless), being unacceptable (e.g., unwanted, damned, wrong), or malevolence (e.g., malevolent, hostile, unfaithful), negative words not related to the BPD schemas but related to stinginess (e.g., inflexible, greedy, stingy), or to physical threat (e.g., pain, cancer, accident), neutral words referring to abstract 


\section{SUCCESSFUL PSYCHOTHERAPY REDUCES HYPERVIGILANCE IN BPD}

academic themes (e.g., abstract, culture, theory), or house interiors (e.g., curtain, table, sofa). The stimulus types consisted of 12 different words each and were matched on mean number of syllables $(M=2.4$, range $(1-3))$. Words were not matched on frequency of use because this has been shown not to influence response latencies (Foa \& McNally, 1986; McNally et al., 1990; Watts et al., 1986). Words had been systematically selected on degree of specificity and emotional valence from a larger pool of words from previous research, lexicons, and the authors' own imaginations. Specificity of the person related stimuli (not house interior words) was judged by 7 therapists with clinical and theoretical knowledge of BPD related schemas. $69 \%-92 \%$ of the stimuli were correctly classified. Ten non-therapists judged the emotional valences of the stimuli. Schema related and schema unrelated stimulus types were rated equally negative, $t(9)=0.72, n s$, and both more negatively, $t(9) \mathrm{s}=9.58, p s<.005$, one-tailed, than the neutral type. Separate emotional stimulus types did differ in emotional valence. The following sequential order was obtained which was based on valence ratings: malevolence $<$ physical threat $\leq$ unacceptable $=$ stinginess $\leq$ powerlessness.

Stroop stimuli were presented four times, in four blocks with 84 trials each. Before these blocks, participants practiced with 8 trials with neutral filler words. The stimuli were divided into two sets with 6 words per stimulus type each, and equal mean syllable length per stimulus type. One set was presented supraliminally and the other was presented subliminally. For BPD patients, subliminally presented words at start of treatment were presented supraliminally at the end of treatment, and vice versa. Effects of stimulus set were controlled for by balancing the conditions within and between the groups. Within the blocks, stimuli appeared in random order and in random color, but with maximally two consecutive trials with the same presentation mode (supraliminal, subliminal), stimulus type, or color, and with each color used equally frequently.

\section{Awareness task}

Awareness for subliminally presented stimuli was checked in a detection task with two blocks of 28 trials each which were presented in random order. In each block, participants had to detect whether subliminally presented stimuli were nonsense words (14 trials) or real words ( 14 trials) by pressing one of two buttons of a response box. The 28 real words formed a representative subset (per block two words per stimulus type, and equal number of syllables per stimulus type) of the subliminally presented words in the preceding Stroop 
task. Nonsense words were created from the same letters as the real words, were pronounceable, and matched to the real words on syllable number, and first and last letter. Order of the blocks was balanced within and between the groups. The detection task started with 8 practice trials.

\section{Apparatus}

The task was run on a AMD $500 \mathrm{~Hz}$ PC with an Elsa graphical adapter and a 17 inch Eizo F57 monitor. A Stroop monitor interface connected the computer to a microphone, an error button, and a two-button response box. Software programs running on Windows NT4 randomized and presented the stimuli, and recorded RTs. Accuracy of presenting and recording was $0.5 \mathrm{~ms}$. The room was illuminated indirectly and dimly, at a predetermined level.

\section{Procedure}

All participants were tested individually. Participants were screened in one to six sessions with a biographical checklist, the SCID-interviews, the BPDSI (patients) or BPD-47 (NPs). If participants fulfilled the inclusion criteria, the study was described to them and written informed consent was obtained. Because of problems with development of the task software, 18 patients were tested and again administered the BPDSI when they were already in treatment for 5-7 months. This was, however, still in the first treatment phase and all were still well above the criterion BPDSI score. Six other patients and the NPs performed the Stroop-experiment within one month after the interviews, which was before the treatment started. Participants first completed the STAI. Then, after having received instructions for the Stroop task, participants started with two Stroop task blocks, followed by one awareness task block, a short break, again two similar Stroop blocks, and a second awareness task block. Sixteen of the initial 24 patients completed treatment and were administered the BPDSI and the Stroop-experiment again 30-34 months after start of the treatment. For the patients, the Stroop-experiments formed a part of a larger 3-monthly administered test battery with varying items. They participated in exchange for free treatment. The NPs received 5 euros per hour for their participation. Participants spent about 50 minutes on the Stroop-experiment. 
SUCCESSFUL PSYCHOTHERAPY REDUCES HYPERVIGILANCE IN BPD

\section{Data analysis}

ESI-scores were computed with only the RTs of color-naming responses that were correct (99\% of the RTs) and not shorter than $300 \mathrm{~ms}$ or longer than $3 S D$ above the average per participant and presentation condition $(98 \%$ of the correct RTs). ESI-scores that differed more than 2SD from the mean in their condition of Group, Stimulus, and Presentation were regarded as outliers, and trimmed to that mean score $\pm 2 S D$. ESI-scores of subliminal presentations of participants whose hit rate in the awareness task were $>.50$ and high in comparison to hit rates of the other participants, were excluded.

ESI-scores of supra- and subliminal presentations were analyzed separately with mixed repeated measures analyzes, optionally with follow-up simple or deviation contrasts, or covariates. Moreover, effect sizes (Cohen's $\eta^{2}$ ) were computed for the predicted effects that either were significant $(p<.05)$ or approached significance $(.05<p<.10)$. Note that $\eta^{2}$ of $.01-.05$ is considered as a small effect, $\eta^{2}$ of .06-.13 as a medium effect, and $\eta^{2} \geq .14$ as a large effect (Cohen, 1988).

The first hypothesis (content-specificity) would be supported by an interaction effect of Group and Stimulus Type in the first sub-study with BPD patients showing relatively high scores for the schema related stimuli as compared to the NPs. The second hypothesis (decrease of bias) would be supported by an interaction effect of Group and Time, and possibly Stimulus Type, in the second sub-study with recovered patients showing a decrease or more decrease of (specific) bias from start to end of treatment than non-recovered patients.

\section{Results}

\section{BPD group at start of treatment versus NP group}

\section{Scores on questionnaires}

Scores on the BPD-47, BPDSI, and STAI subscales are listed in Table 3.2. The BPD group at start of treatment showed higher scores than the NP group on all administered questionnaires, $p s<.0005$. 
CHAPTER 3

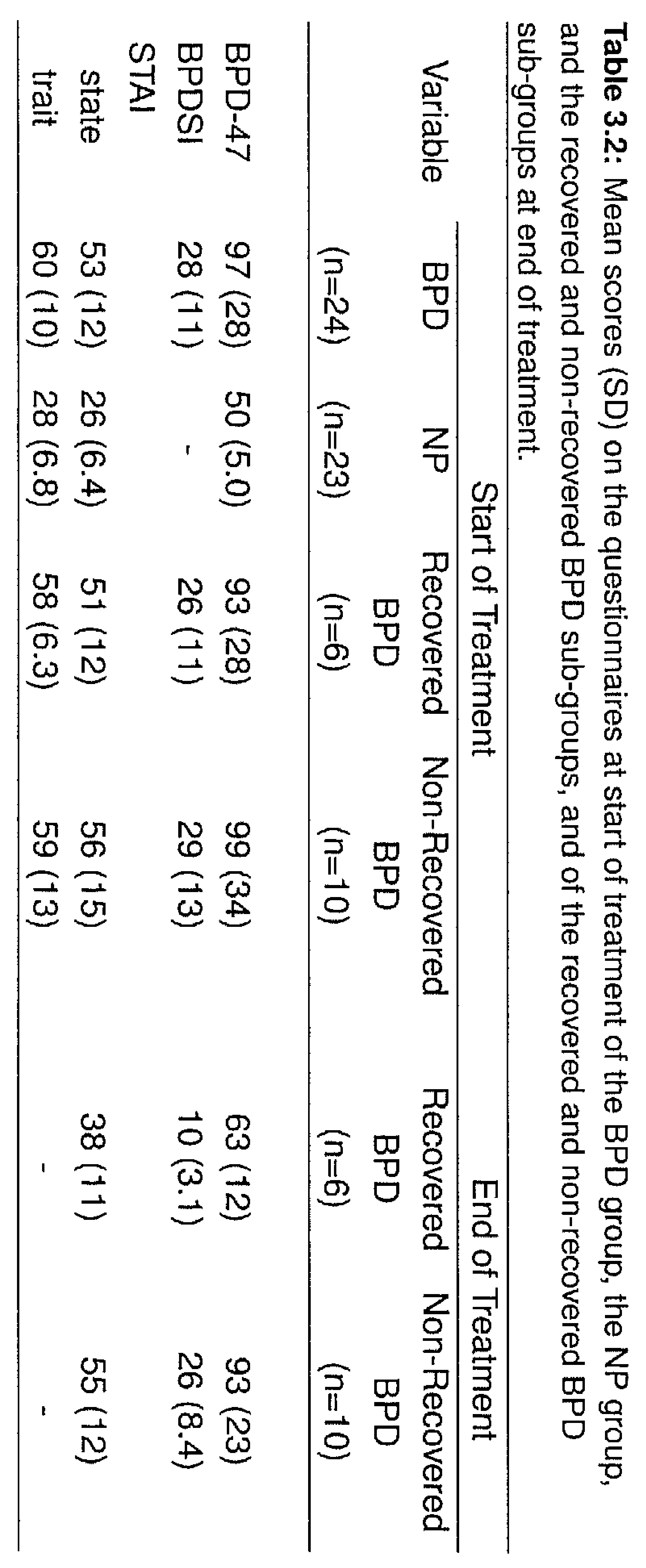




\section{Supraliminally presented stimuli}

ESI-scores for the supraliminally presented stimuli of the BPD group at start of treatment and the NP group are presented in Figure 3.1. Analysis of the ESIscores with the factors Group and Stimulus yielded a significant main effect of Group, $F(1,45)=11.2, p<.005, \eta^{2}=.20$, and trends for a main effect of Stimulus Type, $F_{G G}(2.9,128.6)=2.47, p=.07, \eta^{2}=.052$, and an interaction effect of Group and Stimulus Type, $F_{G G}(2.9,128.6)=2.15, p=.10, \eta^{2}=.046$. BPD patients had higher ESI-scores than NPs. ESI-scores appeared to be relatively low for powerless words, $F(1,45)=5.33, p<.05, \eta^{2}=.11$, and a trend was found for relatively high ESI-scores for stingy words, $F(1,45)=3.03$, $p=.09, \eta^{2}=.063$ (deviation contrasts). Follow-up one-tailed t-tests showed that the interaction effect did not support the first hypothesis: the patients had higher ESI-scores than the NPs for schema related words except powerless words, $t \mathbf{s}>2.13, p \mathbf{s}<.05$, but also for schema unrelated stimulus types, $t \mathbf{s}>$ $2.48, p \mathbf{s}<.01$.

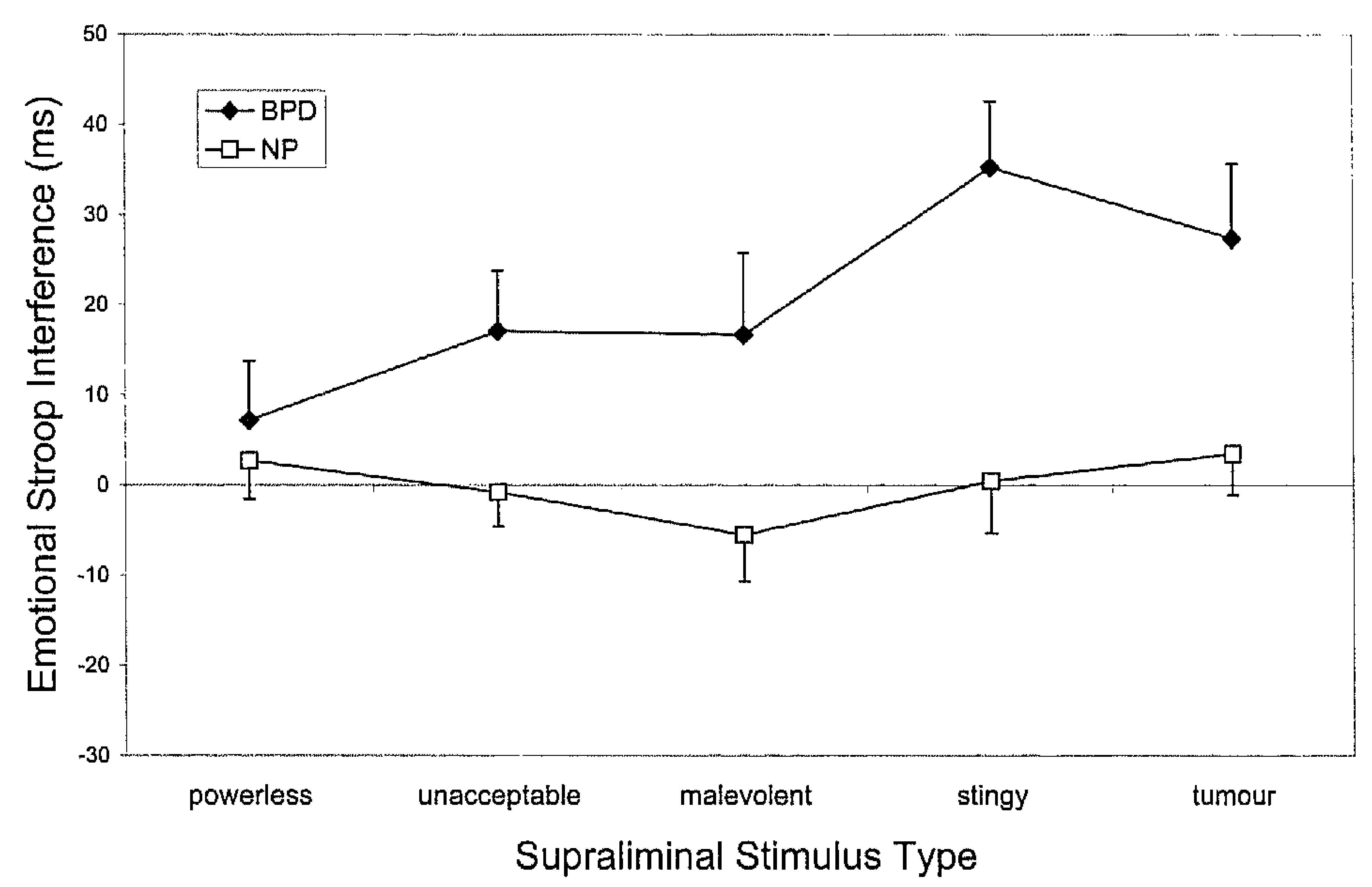

Figure 3.1: Mean interference (ESI) scores (ms) per stimulus type for supraliminally presented stimuli of the BPD $(n=24)$ and NP $(n=23)$ group. 


\section{CHAPTER 3}

Not expecting high ESI-scores for the stingy words in the BPD group, and suspecting that this stimulus type might be related to the unacceptableness schema, we did a correlation analysis within the BPD group. It appeared that ESI-scores for stingy and unacceptable words were indeed relatively highly correlated, $\mu(n)=24\}=.61, p, .015$.$) Some other schema related and unrelated$ stimulus types were also correlated, but less strongly: powerless and physical threat words, $\mu(n=24)=.4 \pi, \mu<.11 .$, and malevolent and physical threat words, $\mu(n=24) \cdots .41, p<.11 \%$.

The ESI-scores were not reanalyzed with STAl-state and -trait scores as covariates because the overlap in scores on these scales of the two groups was too small. Such an ANCOVA would remove too much of the group differences (see, Miller \& Chapman, 2001).

\section{Subliminally presented stimuli}

Mean hit rate in the awareness task $(M=.48, S D=.11$, range (0.15-0.78)) was not greater than $.50, f(46)=-1.08, n . s$, one-tailed. The highest mean hitrate was however rather high and close to the mean hit rate plus 3 SD. ESIscores for the subliminally presented stimuli of the participant with this hit rate were therefore excluded from the analyzes.

Analysis of the ESI-scores with the factors Group and Stimulus Type yielded no significant main or interaction effect of Group and/or Stimulus, $F \mathbf{s}<1.79$, ns. An ANCOVA with STAI-state and -trait scores was again not performed because of small overlap in these scores between the groups. The hypothesis on content specificity was thus also not supported by the group's ESI-scores for subliminally presented schema related and unrelated stimuli.

\section{Recovered patients versus non-recovered patients}

\section{Scores on questionnaires}

Scores on the BPD-47, BPDSI, and STAI subscales of the recovered and nonrecovered patients are listed in Table 3.2. At start of treatment, the (to be) recovered BPD sub-group showed equally high mean scores on all administered questionnaires as the (to be) non-recovered BPD sub-group. At end of treatment, besides showing lower mean scores on the BPDSI, $p<.0005$, the recovered group showed lower scores than the non-recovered group on the STAl-state subscale as well, $p<.05$. 


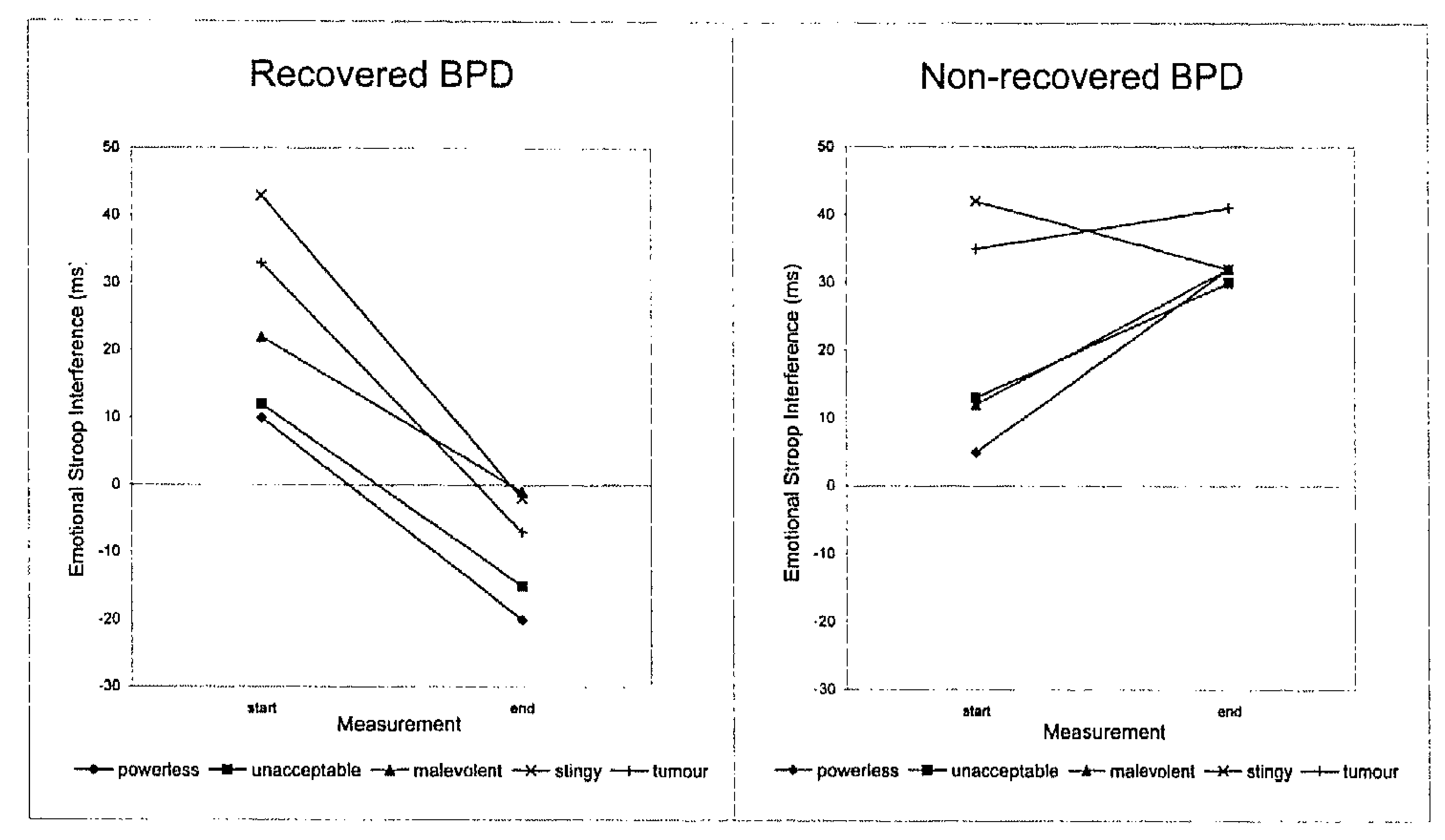

Figure 3.2: Mean interference (ESI) scores (ms) of the recovered BPD $(n=6)$ and nonrecovered BPD group $(n=10)$ for supraliminally presented stimuli at start and end of treatment.

\section{Supraliminally presented stimuli}

ESI-scores for the supraliminal stimuli of the recovered and non-recovered BPD group at start and end of treatment are presented in Figure 3.2. Analysis of these scores with the factors Group, Time, and Stimulus Type yielded a trend for a main effect of Time, $F(1,14)=3.32, p=.09, \eta^{2}=.19$, a main effect of Stimulus Type, $F(4,56)=2.76, p<.05$, eta ${ }^{2}=.16$, and an interaction effect of Group and Time, $F(1,14)=9.14, p<.01, \eta^{2}=.40$. The three-way interaction was not significant, $F(4,56)<1$, ns. Patients showed lower ESIscores at end than at start of treatment. ESI-scores for powerless words were relatively low, $F(1,14)=9.66, p<.01, \eta^{2}=.41$, and a trend was found for relatively high ESI-scores for physical threat words, $F(1,14)=3.14, p=.10$, $\eta^{2}=.18$ (deviation contrasts). The interaction between Group and Time was in the hypothesized direction: patients that were recovered at the end of treatment showed a significant and general decrease in ESI-scores from start to end of treatment, $F(1,5)=11.12, p<.05, \eta^{2}=.69$, whereas ESI-scores of the non-recovered patients did not change, $F(1,9)<1$, ns.

We did not control for state and trait anxiety at start and end of treatment. 


\section{CHAPTER 3}

Covarying for STAI-state and -trait scores at start and end of treatment would remove the group effect because decreases in these scores are too highly correlated to decrease in BPDSI-scores.

It is highly unlikely that the observed effects were caused by medication use. From the patients that had been using medication at start of treatment $(50 \%)$, most did not recover from additive psychological treatment. Most patients that did recover had not been using medication, both at start of treatment $(83 \%)$ and at end of treatment $(100 \%)$. Therefore, use of medication cannot have caused the hypervigilance reduction in the recovered group after treatment.

\section{Subliminally presented stimuli}

Mean hit rates in the awareness task at start of treatment $(M=0.51, S D=$ 0.06 , range (0.43-0.67)) and at the end of treatment $(M=0.50, S D=0.06$, range (0.43-0.61)) were both not greater than $.50, t(15) s<0.78$, $n s$, one-tailed, and highest hitrates were rather low and below the mean hitrate plus 3 SD. All subjects were therefore included in the analysis.

Analysis of the ESI-scores for the subliminally presented stimuli did not support the second hypothesis: the effects with the factor Time were all nonsignificant, $F \mathbf{s}<1.45, n s$. The analysis only yielded a significant main effect of Stimulus Type, $F_{G G}(2.13,29.75)=3.47, p<.05, \eta^{2}=.20$. ESI-scores for subliminally presented powerless words were relatively high, $F(1,14)=5.00, p<.05$, $\eta^{2}=.26$, and those for stingy words were relatively low, $F(1,14)=5.03$, $p<.05, \eta^{2}=.26$.

\section{Discussion}

Several studies (chapter 2 of this thesis; Arntz et al., 2000; Waller \& Button, 1999) have demonstrated that patients with BPD are characterized by selective attention for certain emotional stimuli or by a general kind of hypervigilance. The present study has investigated selective attention in BPD and hypothesized that attentional bias in BPD is schema specific and decreases under the influence of treatments that focus on fundamental change in personality.

The present study again confirmed that patients with BPD are characterized by selective attention for emotionally negative stimuli but, contrary to our hypothesis, the patients with BPD in the present study did not show a content specific bias. Patients with BPD were not found to show selective attention for 
schema related stimulus types only, but also for schema unrelated stimuli, such as stimuli related to being stingy or physical threats. This absence of specific biases is not in line with observations in previous studies (chapter 2 of this thesis; Waller \& Button, 1999). Differences in patient samples and tasks might give some leads for explanations of these divergent findings. The patients in the present study were all outpatients whereas those who showed specific biases were mostly inpatients. Specific biases are perhaps only shown by patients with more severe BPD symptoms. Another difference was that a majority $(71 \%)$ of the patients in the present study had a depressive disorder in contrast to a minority $(44 \%)$ in our previous study (see chapter 2). Depressive mood has been shown to negatively interfere with Stroop effects (Bradley, Mogg, White, \& Millar, 1995), possibly also with content-specific effects. Besides patient factors, task features might also have influenced the findings. An emotional Stroop task presenting a mixture of both negative and positive stimuli (see chapter 2) or a card Stroop (see, Waller \& Button, 1999) might be more sensitive for specific biases. The latter, however, might be more related to late instead of early information processing phases. Specific biases might also be more consistently found when not only the qualifier (e.g., "unacceptable") would be presented, but also the object (e.g., "I"). A primed Stroop task in which the objects are presented prior to the target stimuli (see, Segal \& Gemar, 1997) might be an alternative. Follow-up studies addressing these issues have to be performed, before coming up with theoretical conclusions.

The unexpected bias for stinginess words in BPD might be explained by an excessive sensitivity for rejection and punishment. The correlation of interference scores of the stinginess words with those of the unacceptability words supports this (see also, Waller \& Button, 1999). A study on beliefs suggested that BPD patients are more charaterized by self-rejecting beliefs than by powerlessness and vulnerability beliefs, which were more specific to dependent PD (Arntz et al., 2004). This may explain the rather low interference scores we found for the powerlessness category.

Hypervigilance in BPD was not demonstrated with subliminal stimuli. This is ad odds with the assumption that threat related stimuli are attended to automatically and independently of awareness (e.g., Beck, 1976). The finding furthermore contradicts with biases for subliminal stimuli found in anxiety patients with subliminal tasks (e.g., Lundh, Wikstrom, Westerlund, \& Öst, 1999; Mogg, Kentish, \& Bradley, 1993). Although we did find differential effects for the different types of subliminal stimuli for the whole sample, the stimuli might have 


\section{CHAPTER 3}

been too complex to be processed differentially by the groups.

The most important result of this study was that a reduction of hypervigilance was found in recovered patients but not in non-recovered patients. BPD patients that could be regarded as recovered on the basis of their low BPD symptom level at the end of treatment showed a decrease in hypervigilance to the level of persons without psychopathology, whereas treatment did not reduce selective attention in patients in whom the BPD symptoms sustained from start to end of treatment. Note that reduction of hypervigilance within the group of recovered patients was independent of medication use, but that in the total sample recovery was negatively associated with medication use. Because medication was not randomized, it is unclear what the cause of this association is. Although medication use at start of treatment was not associated with severity (see also, Giesen-Bloo et al., 2006b), patients using medication might be more difficult to treat. Another possibility might be that medication interferes with psychological treatment of BPD. There is one randomized study that indicates that this may be the case (Simpson et al., 2004).

This study cannot rule out that hypervigilance is simply an epiphenomenon instead of a causal or maintenance factor. However, other studies indicate that this is not the case. Two prospective studies have shown that selective attention for (subliminal) threat stimuli predicts non-adaptive responses to subsequent stressful situations (MacLeod \& Hagan, 1992; van den Hout et al., 1995), and another study showed that experimentally manipulated attentional bias influences mood responses to stress (MacLeod et al., 2002). Attentional bias may have a similar role in maintaining BPD.

This longitudinal study demonstrates more strongly than before that hypervigilance is truly characteristic of BPD, which may, at least partly, explain the emotional regulation problems in BPD. This finding is in line with cognitive theories and findings on comorbidity and childhood trauma, stressing the central role of threat bias, anxiety, and hypervigilance in this disorder.

Picturing a patient with BPD as an individual who feels threatened might not only be justified, but also beneficial. Stroop interference can be assumed to tap cognitive processes more directly and independently of demand characteristics than measures depending on introspection and self-report. More than one-third of the treated patients of the sample studied improved on this measure to the level of nonpatients. Realizing that reducing hypervigilance means a change in basic cognitive processes and that BPD has long been considered untreatable, this is very good news for patients with BPD and their therapists, and is promis- 
ing for change oriented clinical treatments. Accepting 'hyper-emotionality"'as an inborn personality characteristic may be premature. Aiming for fundamental change, besides symptom reduction, seems warranted. Anxiety reducing techniques like exposure and cognitive therapy might be helpful, but more fundamental schema change through the therapeutic relationship and processing of childhood traumas are probably the most effective in this respect (see, for example, Arntz, 2004; Young et al., 2003). Future studies should focus on replication and extension (e.g., stability) of these findings, and on how to enlarge success rates and efficiency of change oriented treatments. 


\title{
Chapter 4
}

\section{Not Dichotomous, but Negative Interpersonal Evaluations Characterize Borderline Patients ${ }^{1}$}

\begin{abstract}
Cognitive models explain extreme thoughts, affects, and behaviors of patients with borderline personality disorder (BPD) by specific maladaptive negative schemas and dichotomous thinking, psychodynamic theories ascribe these to splitting. This study hypothesized that making some type of extreme evaluations of others is characteristic of and specific to BPD, and investigated whether these (1) are dichotomous, negative, or split evaluations, (2) limited to specific interpersonal situations, and (3) related to traumatic childhood experiences. A BPD ( $n=18)$, Cluster C PD $(n=19)$, and non-patient group ( $n=17)$ were asked to judge 16 characters portrayed in film fragments in a specific or nonspecific context and with negative, positive, or neutral roles, on specific, nonspecific and neutral visual analogue scales. Schema related negative evaluations of others characterized BPD, particularly negative evaluations of caring characters in an intimate context, and appeared to be related to traumatic childhood experiences, to be independent of depression, and not affectively polarized or split. The results differ from previous studies that found evidence for dichotomous thinking in BPD.
\end{abstract}

\footnotetext{
${ }^{1}$ The content of this chapter has been submitted for publication as Sieswerda, S., Arntz, A., \& Verheul, R., Not dichotomous, but negative interpersonal evaluations characterize borderline patients.
} 


\section{CHAPTER 4}

\section{Introduction}

Various theories have hypothesized that extreme thoughts or representations are central in borderline personality disorder (BPD). Extreme thoughts may lead to extreme affective states and behaviors, like extreme separation anxiety and frantic efforts to avoid abandonment, and anger and displays of temper or (para)suicide attempts. Furthermore, without 'shades of gray', patients with BPD are likely to experience abrupt shifts to an extreme, or from one extreme to another. Extreme thoughts and affects may thus play a major role as well in the instability of relationships, sense of self, and mood of these patients.

Different psychological concepts have been put forward to conceptualize extreme thoughts in BPD. Cognitive models of BPD based on the ideas of Beck (Arntz, 2004; Layden et al., 1993; Pretzer, 1990) hypothesize that extreme thoughts in BPD are manifestations of maladaptive schemas and dichotomous thinking (DT). Schemas are absolute and basic beliefs that organize an individual's perception of the self and others. When maladaptive and active, they can lead to dysfunctional thoughts, feelings, and behaviors, and to systematic cognitive errors that corroborate the schema (Beck, 1976; Layden et al., 1993). BPD patients are thought to have schemas about themselves as being powerless and inherently unacceptable, and about others or the world as being malevolent (Pretzer, 1990). DT is a cognitive error and defined as 'the tendency to evaluate experiences in mutually exclusive categories (...) rather than seeing experiences falling along continua' (Pretzer, 1990, p. 187). Some authors assume a relation between DT and Piagetian preoperational thinking that is thought to characterize children between two and seven years old (Beck, 1976; Layden et al., 1993).

This relation between extreme thoughts and cognitive emotional development is fundamental in object-relation theories of borderline patients ${ }^{1}$. These models conceive extreme thoughts and behaviors of borderline patients as manifestations of splitting, i.e., the complete separation of good from bad aspects of representations of the self and others, resulting in viewing the self or others as 'all good' or 'all bad' (Adler, 1985; Blatt \& Auerbach, 1988; Kernberg, 1966, 1996). Splitting in borderline patients has been formulated as either a developmental deficit or a defense mechanism (Melito, 1983). 'Developmental

\footnotetext{
${ }^{1}$ Patients with a BPD are in psychodynamic theories conceived of as patients with a 'borderline personality organization'. This category applies to almost all patients with BPD, but can also include patients with other PDs.
} 
splitting' is ascribed to an arrest in an early stage of cognitive development. In this stage, the child is supposed to have outgrown a psychotic personality organization and to have learned maintaining boundary differentiations, but not yet to have acquired total object constancy and the capacity to integrate experiences with opposite valences (Adler, 1985; Blatt \& Auerbach, 1988). 'Defensive splitting' is thought to characterize developmentally more advanced children and patients who have acquired integration capacities but who separate opposite aspects out of fear for destruction of good aspects by bad aspects (Kernberg, $1966,1996)$. The hypothesized outcome of normal development after about the third year of life is a neurotic personality organization in which splitting has been replaced by repression (through which differentiated sets of aspects can be ignored). Kernberg $(1966,1996)$ hypothesizes that borderline patients keep using splitting resulting from an aggressive temperament and/or early childhood traumas.

Extreme thoughts and affects in borderline patients have been investigated with various paradigms. Most studies had a psychodynamic orientation and have investigated spontaneous interpretations of pictures or ink blots, and spontaneous evaluations of intimates. These studies have found that borderline patients showed less integrated, differentiated, and accurate interpretations as compared to patients with neurotic personality organizations (Leichsenring, 1990; Leichsenring, Roth, \& Meyer, 1992; Lerner \& St. Peter, 1984; Westen, Lohr, Silk, Gold, \& Kerber, 1990a), and more or equally cognitively advanced interpretations than patients with psychotic personality organizations (Leichsenring, 1991; Spear \& Sugarman, 1984), which is in accordance with psychodynamic views. Some borderline patients however, appeared to make interpretations that were cognitive-developmentally more advanced than those of neurotic patients (Lerner \& St. Peter, 1984; Westen, Ludolph, Lerner, Ruffins, \& Wiss, 1990c). Borderline patients further repeatedly demonstrated negative or malevolent evaluations and lack of positive evaluations (Ackerman, Clemence, Weatherill, \& Hilsenroth, 1999; Baker, Silk, Westen, Nigg, \& Lohr, 1992; Benjamin \& Wonderlich, 1994; Lerner \& St. Peter, 1984; Nigg, Lohr, Westen, Gold, \& Silk, 1992; Segal, Westen, Lohr, Silk, \& Cohen, 1992; Stern, Herron, Primavera, \& Kakuma, 1997; Stuart et al., 1990; Westen et al., 1990a). Integrating these findings, it has been suggested that what renders borderline patients unique is not a cognitive developmental deficit but negative thinking (NT) or more specifically "an overwhelming tendency to construe interpersonal relations as malevolent" (Stuart et al., 1990, p. 313). 


\section{CHAPTER 4}

Experimental studies inspred by the cognitive model of BPD have looked at various evaluation aspects (extremity, affect polarization, dimensionality , and negativity). stimulus types (persons and things). stimulus characteristics (valence and specificity). and (closed or open) response format. These studies have found evidence for both DT and NT. Evaluatons of specific. interpersonal stimuli (i.e., persons associated with childhood trauma and/or intimate interpersonal relations). given in a closed response format. were found to be more extremely negative and positive for borderline patients than for those of patient and nonpatient controls (Ten Haaf \& Arntz, 2009; Veen \& Arntz, 2000). Evaluations of nonspecific non-interpersonal stimuli (see chapter 5 of this thesis) and/or spontaneous evaluations made in an open response format (Arntz \& Veen, 2001) were not always found to be more extremely negative and positive, although very often more negative. These studies also looked for indices of splitting and their outcomes contradicted the splitting hypothesis as well. Evaluations of borderline patients were repeatedly found to be not less cognitively advanced than those of normal controls (Arntz \& Veen, 2001; Ten Haaf \& Arntz, 2009). and not unidimensional (i.e., 'all negative' or 'all positive' evaluations) but multidimensional (i.e., mixed extreme negative and positive evaluations) (chapter 5 of this thesis; Veen \& Arntz, 2000; Ten Haaf \& Arntz, 2009).

The present study has been designed to further investigate extreme thoughts in BPD. In particular. to what degree BPD patients engage in extreme thinking in positive contexts. DT and splitting have been hypothesized to manifest not only as extremely negative thoughts, but also as extremely positive thoughts and/or affects, as well as to switches between these opposites. Several clinical accounts exists of this kind of extreme thoughts, for example that therapist are devaluated but also idealized by patients with BPD (e.g., Adler, 1985; Clarkin et al., 1999). This idea is so prominent, that it became a part of the DSM criteria set of BPD (American Psychiatric Association, 1994). Extreme positive thoughts and affects in BPD have hitherto not been found in empirical studies, but these studies have often not focused at them. Most studies only included negative or neutral stimuli and/or only investigated the extremity of the evaluations without separating negative evaluations from positive evaluations. $\mathrm{Pa}$ tients with BPD seem more likely to engage in negativistic thinking regarding their evaluations of neutral stimuli, but positive DT might also exist in BPD in certain circumstances. Research on extreme evaluations of positive stimuli in negative contexts have found that patients with BPD did tend to, but not clearly showed more extreme positive judgements of benevolent persons than controls 
(Veen \& Arntz, 2000).

Another goal of this study was to investigate what kind of interpersonal stimuli induce extreme evaluations in BPD and along what type of dimensions. Extreme evaluations of borderline patients have been hypothesized (e.g., Layden et al., 1993) and found (Veen \& Arntz, 2000) to be related to schema-activation. Accordingly, it can be expected that schema related stimuli are evaluated more extremely, particularly on schema related dimensions. It further has been found that patients with BPD not only show extreme evaluations along the good-bad dimension, but also along other dimensions (Benjamin \& Wonderlich, 1994). This finding, like the demonstration of mixed negative and positive evaluations, is not in line with the concept of splitting, but it does accord with the concept of DT (Beck, 1979; Layden et al., 1993; Pretzer, 1990; Veen \& Arntz, 2000), as well as with the view that these patients have difficulty articulating, differentiating, and integrating all kinds of object related aspects (Blatt, Brenneis, Schimek, \& Glick, 1976).

The hypothesis that a history of childhood trauma is related to extreme thinking in BPD was also tested. Childhood traumas are relatively often reported by patients with BPD (Herman et al., 1989; Sabo, 1997; Zanarini, 1997). Maladaptive schemas of patients with BPD (on the self being powerless and unacceptable, and others being malevolent), might be direct cognitive representations of these traumatic experiences. A path described by others is that traumatic childhood experiences result in a general or partially developmental arrest in the pre-operational phase, in which children have not outgrown DT (Layden et al., 1993) or splitting (Kernberg, 1996; Melito, 1983).

In sum, the present study aimed to test the following three hypotheses. First, making some type of extreme interpersonal evaluations in all or certain situations is characteristic of BPD. We explored whether these extreme evaluations can be conceived of expressions of DT, NT, or of splitting, on the basis of extremity, negativity, and affect polarization of the evaluations. Second, borderline patients only show extreme evaluations in specific interpersonal situations (i.e., situations related to BPD schemas and/or intimate relationship problems) indicating schema specific DT or NT, or defensive (instead of developmental) splitting. Third, extreme evaluations in BPD are related to a history of childhood trauma. The hypotheses were tested by looking at evaluations of film characters on visual analogue scales (VASs), a paradigm which has proved to be useful (Veen \& Arntz, 2000). 


\section{CHAPTER 4}

\section{Method}

\section{Participants}

Eighteen patients with BPD, 16 patients with one or more cluster $C$ personality disorders (CPD) (without a cluster A or B PD), and 17 nonpatient controls (NPs) were included in this study. Patients had been referred to outpatient or inpatient mental health centers in the Netherlands. Nine CPD participants had been referred to a center specialized in cluster C PD pathology. Patients who fulfilled the inclusion criteria, were invited to participate. NPs were recruited from the same regions by advertisements among the general public, and by invitation to participate among staff of the collaborating centers. All participants were screened with semi-structured diagnostic interviews (SCID-I and SCID-II or SIDP-IV). NPs had to have no current axis I or axis II disorder, and maximally one axis I disorder in complete remission. Control groups were matched to the BPD group on age and sex. In order to prevent group overlap, control participants meeting more than two BPD DSM-IV criteria were not included. Patients with a bipolar or psychotic disorder, patients and NPs with a mental retardation, or who were intoxicated by alcohol or drugs, were also excluded.

Demographic and diagnostic variables of the sample are presented in Table 4.1. The control groups were comparable to the BPD group on most demographic variables. Frequency of being employed was lower in the patient groups, which might be related to having a PD. The BPD and CPD group were quite similar with regard to axis I pathology. Differences in axis II pathology between the BPD and CPD group were a higher mean number of PDs. In comparison with previous studies, this BPD group showed relatively low BPD Checklist scores ( $M=116 S D=26$ in Arntz et al., 2003) and low numbers of PD disorders ( $M=2.44$ in Veen \& Arntz, 2000), whereas this CPD group showed relatively high BPD Checklist scores ( $M=68 S D=11$ Arntz et al., 2003) and high numbers of DSM-IV axis I disorders and PDs (respectively $M=1.6$ and $M=1.1$ in Veen \& Arntz, 2000).

\section{Design}

The study had a quasi-experimental cross-sectional between-within design. The independent variables were Group (BPD, CPD, NP), Role (specific negative, specific positive, nonspecific negative, nonspecific positive, neutral), and Scale (specific, nonspecific, neutral). The dependent variables were DT score, Split- 
Table 4.1: Demographic and diagnostic variables per group ${ }^{1}$.

\begin{tabular}{|c|c|c|c|}
\hline \multirow{3}{*}{ Variable } & \multicolumn{3}{|c|}{ Group } \\
\hline & BPD & CPD & $\mathrm{NP}$ \\
\hline & $(n=18)$ & $(n=16)$ & $(n=17)$ \\
\hline M Age & $27(7.0)$ & $31(7.7)$ & $30(11)$ \\
\hline$\%$ Female & $83(15)$ & $81(13)$ & $88(15)$ \\
\hline$\%$ Education middle - high level ${ }^{2}$ & $33(6)$ & $38(6)$ & $47(8)$ \\
\hline$\%$ Employed/studying ${ }^{3 *}$ & $33(6)^{a}$ & $38(6)^{a}$ & $88(15)^{b}$ \\
\hline$\%$ Married/cohabiting & $33(6)$ & $44(7)$ & $41(7)$ \\
\hline$\%$ Inpatient & $61(11)$ & $50(8)$ & \\
\hline \multicolumn{4}{|l|}{ SCID-1 $1^{4}$} \\
\hline$\%$ mood disorder & $56(10)$ & $50(8)$ & \\
\hline$\%$ anxiety disorder & $67(12)$ & $75(12)$ & \\
\hline $\mathrm{M} \#$ (current) disorders & $2.0(1.4)$ & $2.0(1.3)$ & \\
\hline \multicolumn{4}{|l|}{ SCID-II / SIDP-IV } \\
\hline$\%$ cluster A PD & $6(1)$ & & \\
\hline$\%$ cluster B PD, not BPD & $6(1)$ & & \\
\hline$\%$ cluster $C P^{*}$ & $39(7)$ & $100(16)^{5}$ & \\
\hline M\# disorders* & $1.7(0.8)$ & $1.2(0.4)$ & \\
\hline M \# BPD criteria* & $5.7(1.3)^{a}$ & $0.4(0.7)^{b}$ & $0.1(0.2)^{b}$ \\
\hline M BPD Checklist* 6 & $108(22)^{a}$ & $95(25)^{a}$ & $52(6.2)^{b}$ \\
\hline \multicolumn{4}{|c|}{ 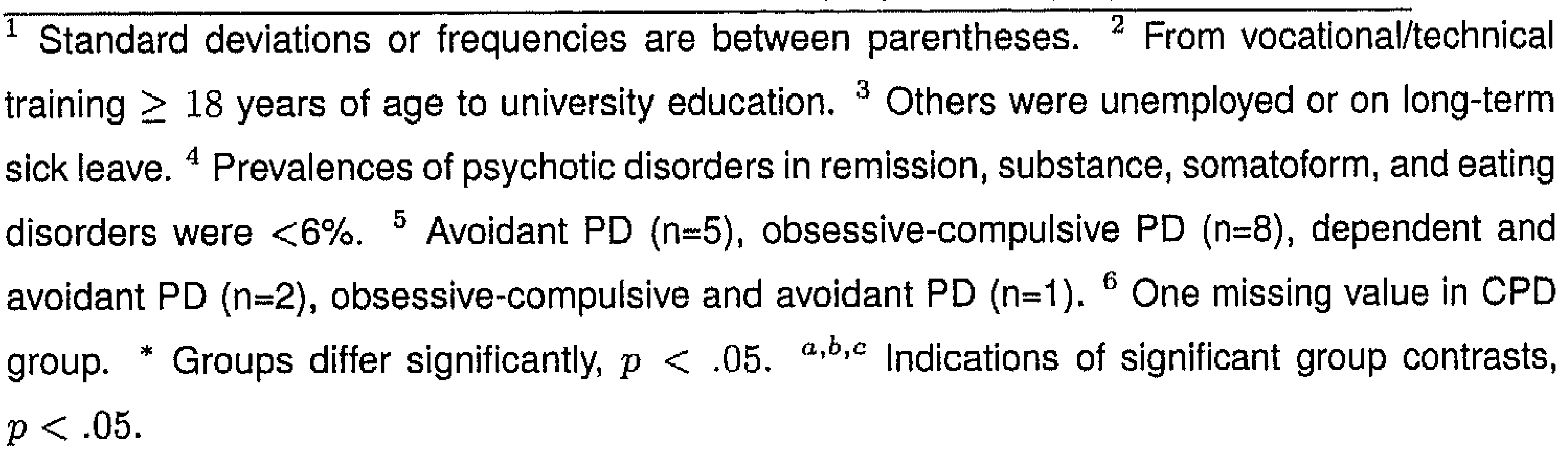 } \\
\hline
\end{tabular}




\section{CHAPTER 4}

ting score, and NT score. Scale had three levels (specific, nonspecific, neutral) for the DT scores, and two levels (specific, nonspecific) for the Splitting and NT scores.

\section{Material}

\section{Diagnostics}

DSM-IV axis I and II diagnoses were assessed with the Dutch-language versions of the SCID-I (First et al., 1997; Groenestijn et al., 1999) and the SCID-II (First et al., 1994; Weertman et al., 2000) or the SIDP-IV (Pfohl, Blum, \& Zimmerman, 1996; Jong de, Derks, van Oel, \& Rinne, 1996). These semistructured interviews are adequately reliable (Damen, de Jong, \& van der Kroft, 2004; Maffei et al., 1997; Ventura, Liberman, Green, Shaner, \& Mintz, 1998; Weertman et al., 2003). Six BPD patients and 12 CPD patients of one center were administered the SIDP-IV as part of that center's admission interview, all other participants were administered the SCID-II. Current severity of BPD symptoms was measured with the BPD Checklist (Arntz \& Dreessen, 1992), a self-report questionnaire consisting of 475 -point Likert scale items on which participants can indicate to what degree they are currently troubled by a wide range of DSM-IV BPD symptoms (range: 5-235). The BPD Checklist has a very good internal consistency (Cronbach's $\alpha=.94$ ) (Arntz et al., 2003; GiesenBloo et al., 2006a).

\section{Characters}

Participants evaluated 16 characters representing ten roles. Table 4.2 presents an overview of the roles, characters, and film clips. Roles could be subdivided according to their specificity (specific, nonspecific, neutral) and emotional valence (negative, positive, neutral). Roles were specific when an emotional interaction in a primary support group, i.e., a partnership, a parent-child interaction, or an intimate friendship, was prominent in the film clip. These type of roles were thought to induce schema related and/or childhood trauma related thinking in borderline patients. Two specific roles were ambiguous, the negative or positive intentions of these (perpetrator or lover) roles were less obvious. Each film clip was five to ten minutes long and could be followed without prior knowledge. The characters represented the role types reasonably accurately, as eight independent judges ascribing role types to the characters, using definitions of the five 
role types and a free-response option, reached a mean percentage correct of $73 \%$.

\section{Emotional state}

Participants rated their emotional state on five $100 \mathrm{~mm}$ VASs (i.e., happy, sad, anxious, angry, aroused). Ratings were scored afterwards from 0 (not at all) to +100 (extremely). The ratings of happiness and mirrored ratings of the three negative emotions were averaged to create one composite emotion score, besides one arousal score.

\section{Character evaluations}

Participants evaluated each character on 26 trait VASs. There were three scale types: (1) 12 specific traits related to the BPD schemas (e.g., powerless - powerful, bad - good, rejecting - accepting), (2) seven nonspecific traits not related to the BPD schemas, with a negative and positive pole (e.g., stingy - generous, untidy - tidy), and (3) seven neutral traits not related to the BPD schemas, with neutral poles (e.g., persistent - flexible, contemplative - action taking). Evaluations were scored afterwards from -50 (pole with negative valence or randomly chosen neutral pole) to +50 (pole with positive valence or the other neutral pole).

A separate nonpatient group $(\mathrm{N}=10)$ rated the scales on relatedness to the BPD schemas and the poles of the scales on emotional valence, in order to check the validity of the scale types. Mean ratings showed that the specific scales were significantly stronger related to the BPD schemas than nonspecific and neutral scales, $t(9) s>3.90, p s<.005$, and differences between the emotional valences of the poles of the specific and nonspecific scales were significantly larger than those of the neutral scales, $t(9) s>6.73, p s<.0005$.

Evaluations of the characters were converted to (1) DT scores, i.e., means of the absolute evaluation scores on the nonneutral and neutral VASs, or $\frac{\sum_{i=1}^{n}\left|V A S_{i}\right|}{n}$, (2) NT scores, i.e., the negative means of the (not absolute) evaluation scores on the nonneutral VASs, or $\frac{-\sum_{i=1}^{m} V A S_{i}}{m}$, and (3) Splitting scores, i.e., absolute means of the (not absolute) evaluation scores on the nonneutral VASs, or $\frac{\left|\sum_{i=1}^{m} V A S_{i}\right|}{m}$ (see, Veen \& Arntz, 2000, for a detailed explanation of these calculations). The score types were calculated for the evaluations on each scale 
CHAPTER 4

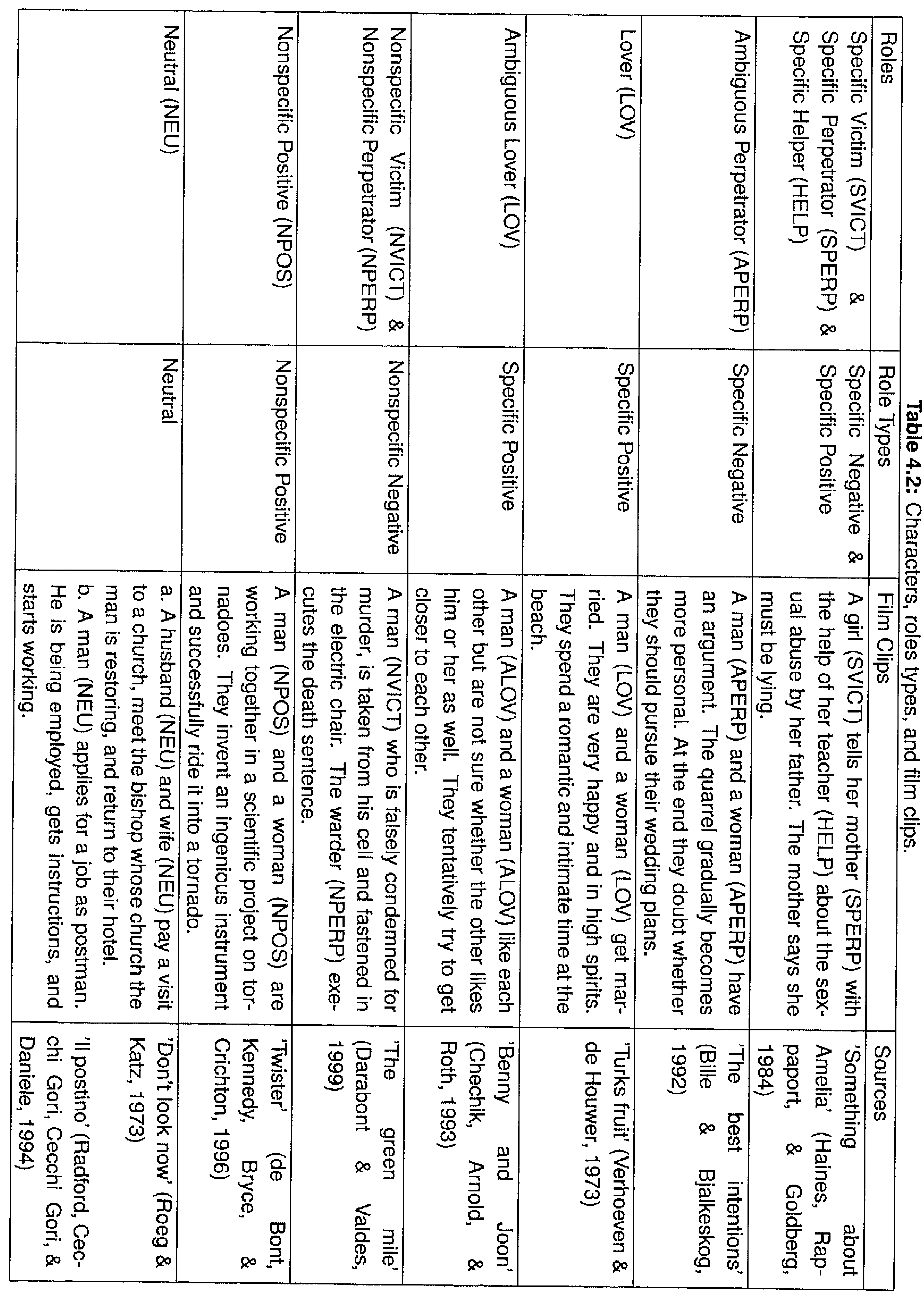


type of the specific negative, specific positive, nonspecific negative, nonspecific positive, and neutral roles, as well as for the separate specific roles.

\section{Childhood trauma scores}

Traumas during childhood were assessed with the Structured Childhood Trauma Interview (VBG) (van den Bossche et al., 1999) rating occurrences of specific events of sexual abuse, physical and neglect, emotional abuse and neglect, and several objective aspects of each event type. Events and their aspects are rated a priori according to degree of damaging potential. For example, abuse received higher ratings when the abusers were more close to the participant, when the abuse took place at a lower age of the participant, with higher frequencies or longer duration. Summing the ratings resulted in three composite scores for severity of childhood trauma: sexual abuse (range (0-47)), physical abuse and neglect (range (0-76)), and emotional abuse and neglect (range (097)). Number of reported events and (comparable) composite scores have been found to show a high 27-months test-retest reliability (Kremers, Spinhoven, \& Van der Does, 2004).

\section{Procedure}

The screening and invitation procedure differed somewhat across the centers from which the patients were recruited. Patients of one center had already been administered the SCID-I and SCID-II as part of their admission interview, before being informed about the study and invited to participate. Patients of another center had already been interviewed with the SIDP-IV and were thereafter informed, invited, and additionally administered the SCID-I. All other participants were informed and invited first, and were then administered the SCID-I and SCID-II. All participants signed a written consent. Participants who had been partly screened by the center's admission interview gave permission to include the data of these interviews in the study. The screening took one to four sessions.

The experiment was performed in a next session. Participants firstly filled out the BPD Checklist and received written instructions and broad information on the goal and procedure of the experiment. The experiment began by participants indicating their baseline emotional state on VASs. Participants were then presented the first film clip. After this, they respectively assessed their emotional state again, gave their spontaneous evaluations of one to three specified 
CHAPTER 4

characters (for another study), and their evaluations of one to three specified characters on the VASs. This procedure was repeated with the second through the eighth fim clip. with a short break between the fourth and fifth clip. Film clips with specific and nonspecific, negative. positive. and neutral, and ambiguous and unambiguous themes were alternately presented. The order was randomily chosen from a set of eight orders in which each film clip was presented once in each ranking. Each order was used at least two times in each group of participants. The experiment ended with the administration of the VBG and a debriefing NPS received a small compensation of 5 EUROs per hour. Patients were offered the possibility to be informed about the results of the diagnostic interviews.

\section{Statistical analyzes}

Demographic and diagnostic group differences were analyzed with ANOVAs, $t$-, and $y^{\prime}$-tests. Differences in emotional states from baseline to post-film measurement were analyzed with one-tailed or, if indicated, two-tailed $t$-tests.

We investigated the DT, NT, and Splitting scores with ANOVAs and onetailed follow-up t-tests. First, scores were analyzed for levels of Group (BPD, CPD, NP). Role Type (specific negative, specific positive, nonspecific negative, nonspecific positive, neutral), and Scale (specific, nonspecific, and/or neutral). Relevant effects were further investigated. We focused on the Group contrasts: $B P D$ vs $C P D$, and $B P D$ vs NP. In case the BPD group would show differential DT, splitting and/or NT, DT and Splitting scores of the BPD group would be compared by an ANOVA with the factors Score (DT, Splitting), Group, Role Type, and Scale. A main or interaction effect of Score with higher DT than splitting scores would indicate mixed negative and positive evaluations, instead of totally negative or totally positive evaluations (see also, Veen \& Arntz, 2000). Finally, the composite scores for childhood trauma were added as independent variables together with having a BPD and CPD diagnosis in regression analyzes with (DT, Splitting, and/or NT) scores and role types for which a significant contrast with BPD were found, as dependent variables.

The scores had skewed distributions and outliers. Kolmogorov-Smirnov tests for the distributions of the three evaluation scores were however nonsignificant, and reanalyzes with nonparametric ANOVAs of the median group scores (Wilcox, 1997) yielded similar results as analyzes with standard ANOVAs. Outliers in the relation between childhood trauma scores and DT, Splitting and/or 
NT scores were checked by visually inspecting the scatter plots.

\section{Results}

\section{Manipulation Check}

The film clips seem to have induced the intended emotional states. After the negative clips, participants reported lower emotion scores than at baseline, $t(50)=-10.5, p<.0005$, although similar arousal scores as at baseline, $t(50)=1.17, n s$. Positive clips were followed by more positive emotion scores than at baseline, $t(50)=2.72, p<.005$, and lower arousal scores than at baseline, $t(50)=-2.87, p<.005$. After the neutral film clips, emotion scores were equal to baseline, $t(50)=0.71, n s$ (two-tailed), and participants again reported less arousal than at baseline, $t(50)=-3.62, p<.001$ (two-tailed). Post-hoc explanations for finding no differences in arousal after the negative film clips and lower arousal after the positive and neutral film clips are high scores for arousal at baseline, possibly resulting from apprehension, and relaxation after the positive and neutral clip.

We largely succeeded in selecting nonspecific film clips that were as emotional as the specific films. Decreases and increases in (positive) emotion scores after respectively the negative and positive nonspecific clip were not smaller than those after the negative and positive specific clips, $t(50) s<1.29$, $n s$; decrease in emotion score after the negative nonspecific clip was even larger than after the negative specific clip $t(50)=4.58, p<.0005$ (two-tailed). Increase in reported arousal after the negative nonspecific clip also matched those after the negative specific clips, $t(50)=.20, n s$. Only decreases in reported arousal after the positive films were lower after the nonspecific clip than after the specific clip, $t(50)=2.37, p<.05$ (two-tailed).

\section{Characteristics of extreme evaluations}

\section{DT scores}

The ANOVA of the DT scores yielded one significant effect involving Group: a three-way interaction effect of Group, Role Type, and Scale, $F_{G G}(12.1,291)=$ $2.38, p<.01$. Other effects with the factor Group were nonsignificant: Group, $F(2,48)=0.39$, ns; Group $\times$ Role Type, $F_{G G}(5.8,139)=0.82$, ns; Group $x$ Scale, $F_{G G}(3.5,83)=1.57$, ns. We examined the three-way interaction with 


\section{CHAPTER 4}

separate ANOVAs for the three levels of Scale. A significant interaction of Group and Role Type, $F_{G G}(6.4,153)=2.30, p<.05$, was found to. scores for specific scales, but not for the DT scores for the other scales the specific scales, BPD patients showed higher DT scores for the nonspas negative roles $(M=33, S D=8)$ than the CPD patients $(M=28, S I)$ $t(32)=1.81, p<.05$, but not compared to the NP group $(M=31, s l$ The BPD group thus did not show the hypothesized contrasts with the NP $9 n_{\text {; }}$ and for the specific roles. Mean DT scores for the role types and specific se are presented in Figure 4.1.

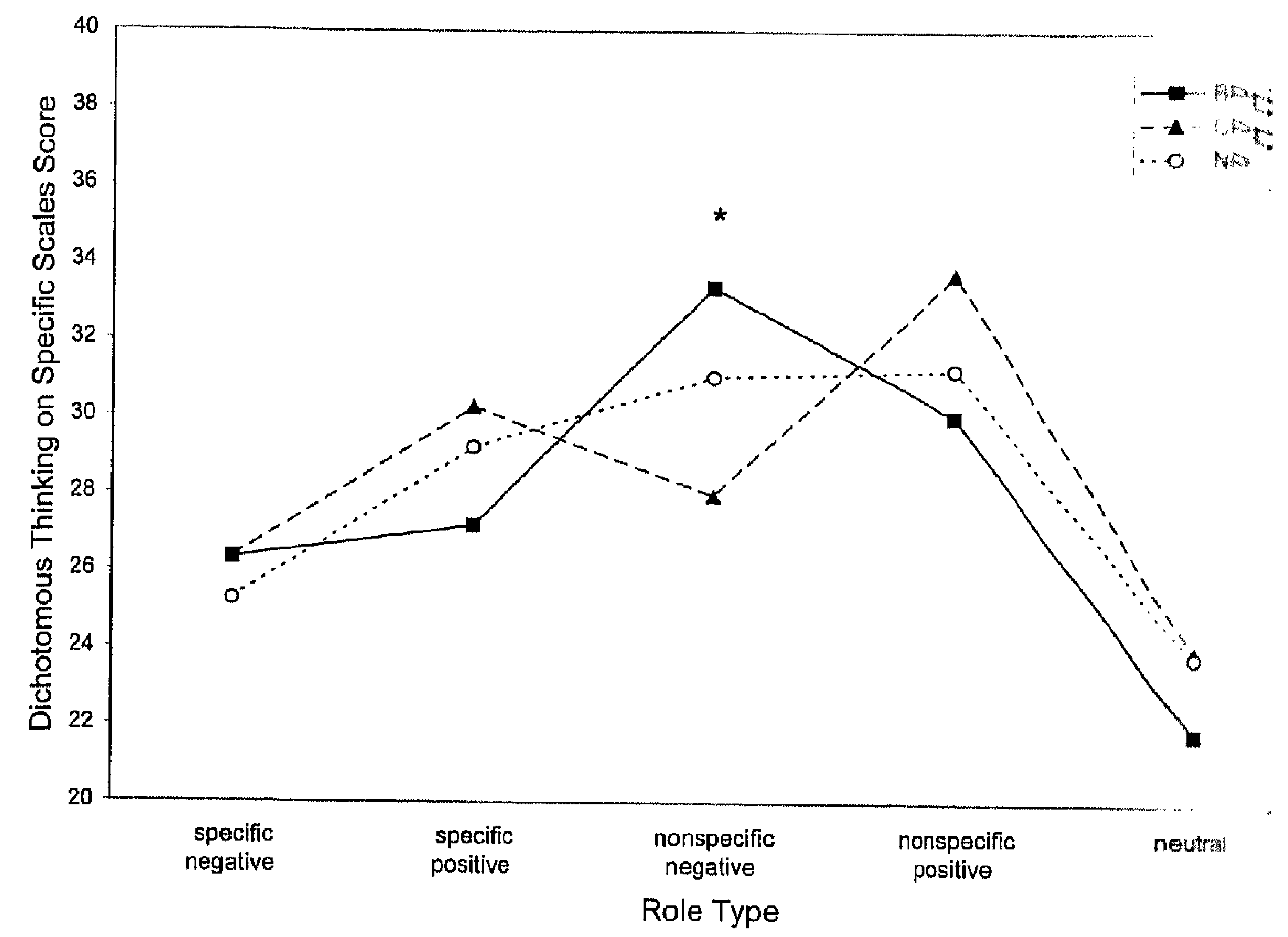

Figure 4.1: Mean DT scores for the role types and specific scales of the BPD $(n=1$ CPD ( $n=16)$, and NP Group ( $n=17)$.

In order to investigate whether the nonsignificant contrasts were due to I atively low DT scores of the BPD and/or high DT scores of the control groups compared the scores found by Veen and Arntz (2000), we compared the grou overall DT scores for evaluations of five roles (specific perpetrator, ambiguc. perpetrator, specific victim, helper, neutral) and on scales (specific, neut' these studies had in common. The BPD group of the present study did sh 
a somewhat but not significantly lower overall DT score $(M=26.3 S D=5.6)$ than the BPD group of the previous study $(M=28.2 S D=8.5), t(25)=-0.75$, $n s$. The overall DT scores of the present CPD $(M=28.0 S D=7.1)$ and NP group ( $M=25.5 S D=7.4$ ) however were respectively significantly and nearly significantly higher, $t \mathbf{s}>1.41, p<.08$, than those of the previously investigated groups (CPD: $M=22.1 S D=6.6$; NP: $M=22.4 S D=4.8$ ).

\section{NT scores}

NT scores for the groups and the role types are presented in Figure 4.2. The ANOVA of the NT scores revealed an interaction effect of Group and Role Type, $F_{G G}(6.3,150)=2.37, p<.05$. Other effects involving Group were nonsignificant: Group, $F(2,48)=1.64$, ns; Group $\times$ Scale, $F(2,48)=.31$, ns; Group $\times$ Role Type $\times$ Scale, $F_{G G}(6.3)=$,1.20 , ns. It appeared that the BPD group showed higher NT scores for the specific negative roles, $t(33)=3.65$, $p<.0005$, and the neutral roles, $t(33)=2.10, p<.05$, as compared to the NPs, and for the specific positive roles as compared to the NP group, $t(33)=1.83, p<.05$, as well as the CPD group, $t(32)=2.71, p<.01$. The BPD group thus showed more NT for film characters with specific and neutral roles, with NT for characters with specific positive roles being specifically high in BPD.

To test whether the higher NT scores for the neutral roles of the BPD group was due to two of the three neutral characters being partners in an intimate relationship, we performed another ANOVA of the NT scores with the factors Group, Neutral Character Type (intimate partner, postman), and Scale. This analysis however did not yield effects of Neutral Character Type and Group, $F \mathbf{s}<0.30, n s$, that would support such an explanation.

Negative interpersonal evaluations are thought to be characteristic for patients with BPD as well as for depressive patients (e.g., Beck, 1979). We therefore performed three linear regression analyzes with fulfilling criteria for BPD and for a current depressive episode as independent variables, and as dependent variable NT for specific negative roles, specific positive roles, or the neutral role. Having BPD appeared to be significantly and positively related to NT for the specific negative, $\beta=.35$, and positive roles, $\beta=.38, p \mathbf{s}<.01$, and nearly significantly for the neutral role, $\beta=.19, p=.10$. Coefficients for currently going through a depressive episode were not significant for any of the role types, $-.16<\beta s<.15, p s>.32$. This indicated that the group contrasts for NT thinking could not be attributed to depression. 


\section{CHAPTER 4}

In order to explore whether the BPD patients evaluated some specific roles as more negative than others, we examined whether an ANOVA of the NT scores with the factors Group, Specific Role (perpetrator, ambiguous perpetrator, victim, ambiguous lover, lover, helper), and Scale, yielded an interaction effect between Group and Specific Role. This appeared to be the case, $F(10,255)=2.60, p<.01$. The BPD group showed higher NT scores for the perpetrator and ambivalent perpetrator roles compared to the NP group, $t \mathbf{s}(33)>3.12, p s<.005$, and for the lover and ambiguous lover roles as compared to both the CPD and NP group, $t \mathrm{~s}>1.81, p \mathrm{~s}<.05$. NT scores of the three groups did not differ for the victim and helper role. Mean NT scores of the groups for all roles are presented in Figure 4.2.

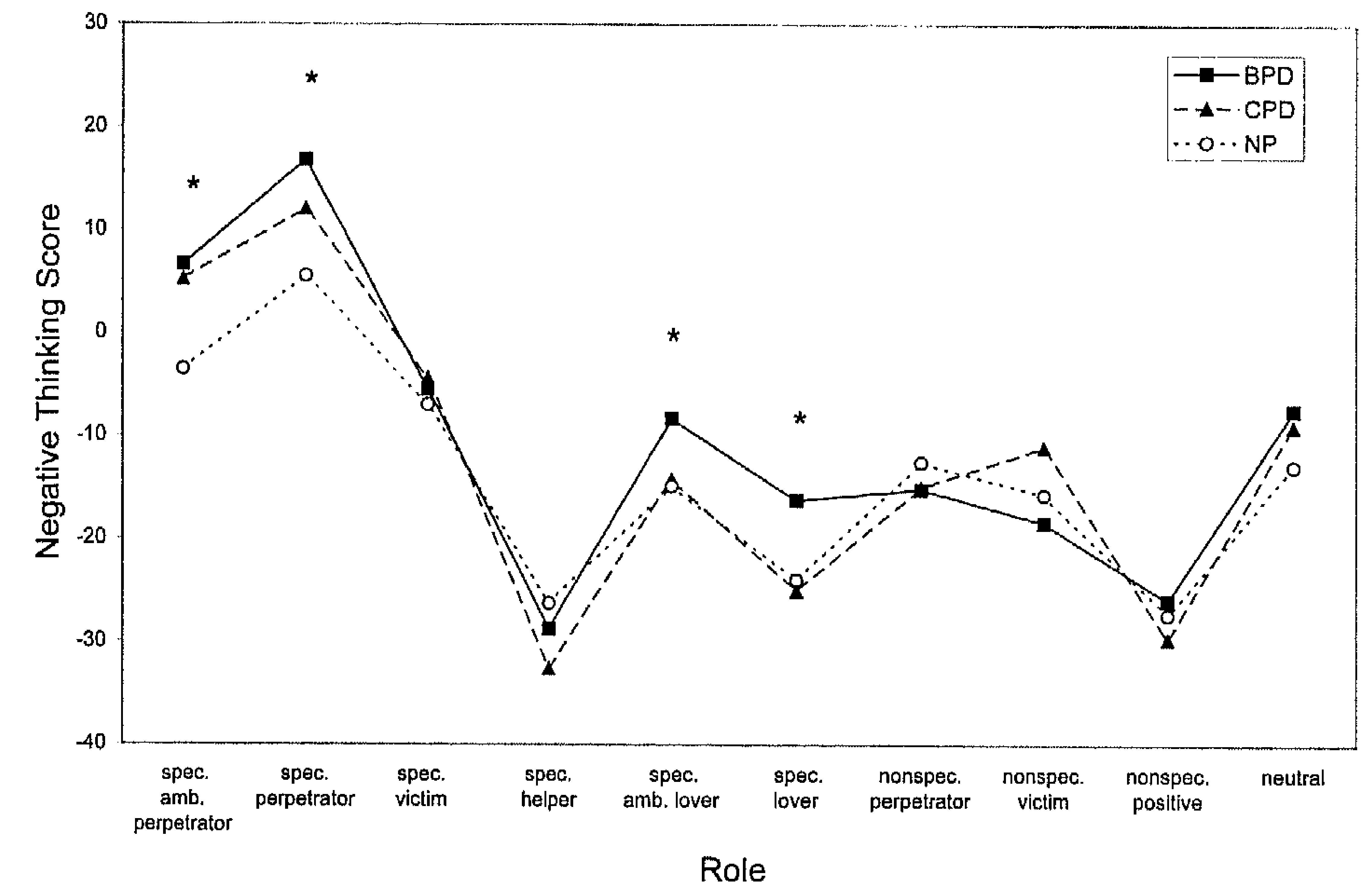

Figure 4.2: Mean NT scores for all roles of the BPD $(n=18)$, CPD $(n=16)$, and NP Group $(n=17)$.

\section{Splitting scores}

The ANOVA of the Splitting scores did not reveal any significant main or interaction effect of Group, $F \mathbf{S} \leq 1.25, n s$. These results imply that neither the BPD 
group, nor patients with higher BPD symptom levels showed higher Splitting scores than the other groups.

\section{DT vs Splitting scores}

We performed an ANOVA of the DT and splitting scores of the BPD group, to test whether higher DT scores in patients more BPD pathology and higher NT scores in the BDP group resulted from a complete polarization of all scores into the negative or positive direction ('all bad' or 'all good' thinking). This analysis resulted in a main effect of Score, $F(1,17)=70.3, p<.0005$, with higher DT scores $(M=22.2, S E=1.48)$ than Splitting scores $(M=14.1, S E=14.1)$. DT scores also appeared significantly higher than the Splitting scores in t-tests per role, $p s<.005$. These outcomes indicate that the dichotomous evaluations were not affectively polarized and the negative evaluations of the BPD group were predominantly negative, but not on every VAS.

\section{Relation with childhood trauma}

VBG composite scores are presented in Table 4.3.

Table 4.3: Mean composite scores on the VBG subscales per group ${ }^{1}$.

\begin{tabular}{lccc}
\hline \multirow{2}{*}{ Variable } & \multicolumn{3}{c}{ Group } \\
\cline { 2 - 4 } & $\begin{array}{c}\text { BPD } \\
(n=18)\end{array}$ & $\begin{array}{c}\text { CPD } \\
(n=15)\end{array}$ & $\begin{array}{c}\text { NP } \\
(n=16)\end{array}$ \\
\hline Sexual abuse* $^{*}$ & $6.6(9.6)^{a}$ & $5.4(8.5)^{a}$ & $0.3(1.3)^{b}$ \\
Physical abuse and neglect* $^{*}$ & $18(11)^{a}$ & $9.3(12)^{b}$ & $1.6(3.5)^{c}$ \\
Emotional abuse and neglect* $^{*}$ & $34(15)^{a}$ & $22(14)^{b}$ & $2.1(5.9)^{c}$ \\
\hline
\end{tabular}

${ }^{1}$ Standard deviations are between parentheses. One missing value in CPD and NP group.

${ }^{*}$ Groups differ significantly, $p<.05{ }^{a, b, c}$ Significant group contrast, $p<.05$.

We investigated the relations between traumatic childhood experiences and NT by running three regression analyzes with the VBG sexual abuse score, VBG physical abuse or neglect score, VBG emotional abuse or neglect score, $B P D$ diagnosis (BPD, not BPD), and CPD diagnosis (CPD, not CPD) as independent variables and the NT score for the specific negative, specific positive, or neutral role type as dependent variable. These analyzes revealed a positive relation $(\beta=.38), p<.05$, between VBG score for severity of sexual childhood abuse and NT score for the specific negative role type. The relation between 


\section{CHAPTER 4}

BPD diagnosis and NT score for the specific negative role type remained significant in this analysis $(\beta=.57), p<.005$. Relations between VBG score and NT scores for other combinations of VBG subscale and role type were not significant $(\beta \mathbf{s}<.23), p \mathbf{s}>.24$.

\section{Discussion}

This study investigated interpersonal evaluations in BPD, in particular what kind of extreme interpersonal evaluations are characteristic of BPD. Patients with $B P D$ were found to be characterized by more negative interpersonal evaluations. These negative evaluations were independent of currently experiencing a depressive episode. This finding is consistent with other empirical studies. An extensive row of studies of the group of Westen (Stuart et al., 1990; Westen et al., 1990a; Westen, Ludolph, Block, Wixom, \& Wiss, 1990b; Westen et al., $1990 \mathrm{c}, 1990 \mathrm{~d}$ ) in which object relations in BPD were investigated with various projective stimuli, similarly converged towards the conclusion that borderline patients tend to see others as malevolent. A study on facial expression in BPD demonstrated a persistent reduced degree of emotionally positive facial expressions in patients with BPD in comparison with depressive and healthy controls (Renneberg, Stäbler, Fiedler, Weisbrod, \& Kaiser, 2008). A previous study in which patients with BPD also evaluated film characters (Veen \& Arntz, 2000) only assessed DT. Perhaps NT was there as prominent as in the present study.

The absence of a clear difference in DT between the BPD group and the controls is inconsistent with previous studies (Veen \& Arntz, 2000; Ten Haaf \& Arntz, 2009). Borderline patients did not judge others more dichotomously, i.e., with more extreme negative and positive part-evaluations than control participants. The difference between the contrasts for DT in this study and those in Veen and Arntz (2000) appeared to be mainly due to relatively high scores for DT of the control groups in the present study. A high number of BPD features of the PD control participants, who showed relatively high scores on a BPD symptom inventory (BPD Checklist), may have accounted for the absence of contrast in DT between the BPD and CPD group. Maybe, DT is not characteristic of patients with BPD only, but also for patients with other serious PDs. CPD participants who were interviewed with the SIDP-IV $(n=9)$ may also have had unnoticed BPD features because this interview describes less criterion behaviors than the SCID-II. If DT is characteristic of lower cognitive levels as well, lower intelligence of the healthy participants might explain the similar scores of 
the BPD and healthy control group. Healthy control and BPD participants were matched on educational level, but because educational level probably underestimates intelligence in BPD, matching on educational level may have led to too low intelligence and too high DT scores of the control group. (This explanation does require other factors protecting the control participants for the assumed pathology caused by DT.)

The NT style of the borderline patients did not alternate with (incidental) extremely positive evaluations, for example of characters having a positive role such as a teacher supporting a child, a caring partner or a successful scientist. Evaluations of characters in certain positive roles were actually specifically negative in borderline patients. This result is not in line with some clinical observations (e.g., Adler, 1985; Clarkin et al., 1999) and the DSM criterion of BPD (American Psychiatric Association, 1994) describing instances of idealization in BPD. Maybe there are other factors than stimulus specificity and valence not manipulated in this experiment that cause extremely positive interpersonal evaluations in patients with BPD. Investigations of affect instability in BPD using an experience sampling procedure (Stein, 1996) or assessing the reported intensity to imagined life events (Levine, Marziali, \& Hood, 1997) however similarly found no evidence for extremely positive affects in BPD. Clinical observations may thus be biased as well.

Findings with regard to the form of the interpersonal evaluations suggest that negative evaluations of patients with BPD do not result from (developmental or defensive) splitting. The evaluations of patients with BPD appeared to be non-polarized, i.e., mixtures of negative and positive part-evaluations, instead of 'all bad' or 'all good'. For example, a character was seen as boring, tidy, bad and strong. An absence of affectively polarized evaluations is in accordance with the demonstration of more malevolent evaluations in adolescent than in older borderline patients, implying that NT reflects rather style than a developmental arrest (Westen et al., 1990d). It is further in line with the conclusion that splitting cannot explain the judgments of others of borderline patients, which were found to deviate on a hostile-nonhostile as well as autonomousinterdependent dimension (Benjamin \& Wonderlich, 1994).

The findings did support the idea of NT in BPD being a manifestation of schema related thinking. Evaluations of schema related characters (in intimate interpersonal relationships, such as an abusing partner or a caring partner) were more negative than those of schema unrelated characters (in non-intimate relationships, such as a condemned man or a successful scientist), and this 


\section{CHAPTER 4}

difference tended to be stronger for schema related dimensions of the evaluations as compared to schema unrelated dimensions. Compared to healthy and PD control participants, borderline patients showed particularly less positive evaluations of caring others in an intimate partnership. These findings echo those of another study demonstrating more hostile judgements of borderline patients of particularly their maternal relations, relative to depressive patients (Benjamin \& Wonderlich, 1994). They are also in line with clinical observations. Borderline patients report particular problems in intimate relationships, such as relationships with partners, close friends, parents, and therapists. Invalidation of expressions of love and affection of others and themselves, may be crucial for the difficulties these patients experience. Patients with CPD did not show negative evaluations of caring others which complies with observations of less problematic caring relationships in these patients.

A final outcome concerned the influence of potentially traumatic childhood experiences on interpersonal evaluations. We investigated relations between occurrences of sexual abuse, physical abuse and neglect, and emotional abuse and neglect on the one hand, and NT on the other hand. Negative evaluations of specific negative characters (an emotionally abusing parent or partner) appeared to be partially explained by severity of sexual abuse. It seems likely that individuals with BPD have formed negative schemas on the basis of negative childhood experiences (see also, Arntz, Dietzel, \& Dreessen, 1999) and that activated schemas negatively bias interpersonal perceptions in similar situations. Different from a study on early memories of patients reporting a history of childhood sexual abuse, which were characterized by both more malevolent attributions and less effective helpers (Nigg et al., 1991), childhood trauma did not affect the relation between BPD diagnosis and negative evaluations of specific positive characters (a caring parent or partner) in the present study. Childhood trauma did further not totally remove the relation between BPD diagnosis and negative evaluations of specific negative characters; a finding which is in line with a meta-analysis showing a moderate effect size for the relationship between childhood sexual abuse and BPD (Fossati, Maddeddu, \& Maffei, 1999). Additional factors influencing the generation of negative schemas and negative interpersonal evaluations in BPD might be constitutional factors and/or childhood experiences which were not captured (e.g., chaotic household, invalidating upbringing, unavailable social support) or differentiated (e.g., disrupted maternal attachments) by the childhood trauma interview (see, Downey \& Walker, 1989; Linehan, 1993; Stuart et al., 1990) 
Some limitations may have diminished the external validity of this study. It is not certain whether the DT and splitting scores represent DT and splitting as described in the literature and clinical observations of BPD (e.g., Pretzer, 1990). The extremity of the evaluations may also represent a combination of negative thinking, disintegrated thinking, and/or a hyperbolic expressive style. Another issue is the validity of the calculation of the DT and splitting scores based on the deviation of the evaluations. Computational alternatives could use the variability of the evaluations or the number of deviations of evaluations beyond a certain criterion. Three aspects of the paradigm may further have resulted in less maladaptive evaluations, viz. a relatively high level of structure of the task, the fact that the participants did not interact themselves with the characters, and a relative transparent research goal. Less structure has been shown to result in more polarized (all negative or all positive) part-evaluations (Arntz \& Veen, 2001). The spontaneous evaluations of the participants of this study will therefore also be analyzed in another study. Experiments with real interactions (such as Ten Haaf \& Arntz, 2009) are badly needed, although these may have their own caveats because of the interpersonal hypersensitivity of borderline patients. It would be useful to investigate alternative ways to induce and measure extreme thinking.

We further did not optimally use the possibilities of this paradigm to manipulate and control stimuli in order to maximize internal validity. Firstly, the emotionality and specificity for the negative specific and nonspecific characters, with 'specific' referring to presence of an emotional intimate relationship, were not disentangled. Nonspecific negative characters were evaluated more positively than neutral and specific negative characters. Although the participants did experience the nonspecific negative characters as relatively negative compared to the nonspecific positive characters, unequal valences of the nonspecific and specific negative characters complicates drawing conclusions with regard to the effect of specificity independent of emotionality. A future study should include nonspecific characters with more negative valences, in order to match the specific negative characters. Secondly, the character roles representing the five role types and the specificity conditions were rather heterogeneous. More narrowly defined conditions of roles and specificity (for example perpetrator, victim, or partner in intimate or work related context) might be beneficial.

In sum, in contrast to previous studies, no evidence was found for DT in BPD. Instead, NT appeared to be characteristic of BPD, particularly in the context of positive intimate relationships and partially related to a history of child- 
CHAPTER 4

hood sexual abuse. Whether NT is causal in the instability in BPD is a question for further research. The research underlines the importance of being aware of NT in borderline patients during treatment, but does not support the conception of NT as developmental arrest in a preoedipal phase or as a consequence of traumatic childhood experiences only. 


\title{
Chapter 5
}

\section{Negative Evaluations of Emotional Non-Interpersonal Situations Characterize Borderline Personality Disorder ${ }^{1}$}

\begin{abstract}
According to cognitive models of borderline personality disorder (BPD), an important cause for the instability of patients with BPD is dichotomous thinking (DT). Object-relation theories assume that the similar phenomenon of splitting is central in BPD. Previous studies focusing on interpersonal situations found support for DT being prominent in BPD. The aim of this study was to investigate whether patients with BPD also make use of dichotomous and schemaspecific evaluations in non-interpersonal situations. An experiment was designed in which a frustrating and rewarding situation was induced by computer games that subjects had to play. Participants evaluated both themselves and the games. Patients with BPD $(n=24)$ were characterized by somewhat more extreme game evaluations in the emotionally negative situations than normal controls $(n=25)$, participants with a cluster $C(n=10)$ or an anti-social personality disorder (ASPD) $(n=16)$. Patients with BPD appeared to be characterized best by a general negative evaluative style, more than by DT or splitting. ASPD participants showed a positivity bias in both conditions.
\end{abstract}

\footnotetext{
${ }^{1}$ The content of this chapter has been published as Sieswerda, S., Arntz, A., \& Wolfis, M. (2005), Evaluations of emotional non-interpersonal situations by patients with borderline personality disorder, Journal of Behaviour Therapy and Experimental Psychiatry, 36, 209-225.
} 


\section{CHAPTER 5}

\section{Introduction}

A characteristic feature of patients with borderline personality disorder (BPD) is their instability of affects, self-image and relationships causing problems and conflicts in these domains (American Psychiatric Association, 1994). The Beckian cognitive model of BPD described by Pretzer (1990) and Arntz (2004) attributes this instability to (a) dichotomous (black-or-white) thinking (DT), i.e., evaluating situations in mutually excluding extremes, and (b) incompatible basic beliefs.

It is assumed that borderline patients make extreme, dichotomous evaluations of the world, instead of seeing the world in shades of grey (Arntz, 2004; Pretzer, 1990). Lacking intermediate evaluative categories these patients show extreme emotions and behavior, as well as abrupt shifts from one extreme to the other. Although DT is seen as a common cognitive distortion, which may also be displayed by other patients, this thinking style is thought to be highly prominent in borderline patients.

Besides DT, the basic beliefs or schemas of borderline patients (Arntz, 2004; Pretzer, 1990) are also assumed to increase instability in BPD. According to the model, borderline patients see (i) themselves as powerless and vulnerable, and (ii) as unacceptable, and (iii) the world or others as dangerous and malevolent. The first schema turns them towards others, seeking help and protection, whereas the other schemas drive them in the very opposite direction, that is away from others, preventing punishment and abuse. The combination of dichotomous thinking and the incompatible schemas is very potent and might explain the unstable patterns of BPD.

Most studies on evaluations by patients with BPD (Arntz \& Veen, 2001; Bonis, De Boeck, Lida-Pulik, Hourtané, \& Féline, 1998; Leichsenring et al., 1992; Sheffield et al., 1999; Veen \& Arntz, 2000) support the hypothesis that DT is prominent in BPD. Overall, it was found that borderline patients evaluated others more extremely than clinical and non-clinical controls. Veen and Arntz (2000) found that this was only the case when borderline patients were asked to evaluate persons in a BPD-specific role, such as the role of perpetrator or victim of abuse. Evaluations of persons in an emotional role that was not BPD-specific or a neutral role, were not more extreme than those of others. Possibly, activating BPD schemas is a prerequisite for patients with BPD to think extremely.

The primary aim of this study was to investigate whether the range of situations in which patients with BPD make use of dichotomous thinking also extends 
to non-interpersonal situations. The studies described above focused on interpersonal evaluations. This focus was probably chosen because having unstable interpersonal relationships is a salient feature of borderline patients. Another reason is that object-relation and attachment theories conceptualize extreme evaluations of borderline patients as direct or indirect reiterations of adverse interpersonal childhood experiences (e.g., Fonagy, Target, \& Gergely, 2000; Kernberg, 1996; Sable, 1997). It is however not clear whether DT in BPD is a general characteristic which is applied in frustrating or rewarding interpersonal as well as non-interpersonal situations. Besides having interpersonal problems, patients with BPD also struggle with performing non-interpersonal tasks such as studies, jobs, and household tasks. This kind of problems might stem from extreme non-interpersonal evaluations.

A secondary aim of this study was to investigate the way DT in BPD manifests itself in non-interpersonal situations, and how to exactly conceptualize non-interpersonal DT: as (schema related or unrelated) cognitive distortions, as the defense mechanism 'splitting', or as negative thinking. Instead of a cognitive distortion, object-relation theories (e.g., Kernberg, 1966, 1996) conceive extreme evaluations of borderline patients as expressions of 'splitting', a defense mechanism thought to separate good and bad aspects of self- and otherrepresentations. It is assumed that split evaluations (1) are unidimensional or univalent, i.e., all good or all bad, and (2) can be extremely positive (idealization) as well as extremely negative (devaluation). Studies focusing on interpersonal evaluations, found evidence for both unidimensional DT (Arntz \& Veen, 2001), as well as multidimensional or multivalent (for example, very good and very bad) DT (Bonis et al., 1998; Sheffield et al., 1999; Veen \& Arntz, 2000). According to Arntz and Veen (Arntz \& Veen, 2001) response format might explain these ambiguous findings. A structured response format might protect patients with BPD from primitive, unidimensional thinking levels and help them to perform on higher levels than usually in normal life. Extreme positive thinking was not found in these studies, but this might be caused by having used only neutral and negative stimuli.

Extreme evaluations in borderline patients might also be expressions of a negative thinking style or negativity bias. Several studies, mostly using projective stimuli, found that a lower affect-tone of the object world was the most specific feature of borderline patients spontaneous narratives or evaluations (Arntz \& Veen, 2001; Kurtz \& Morey, 1998; Nigg et al., 1992; Segal et al., 1992; Spear \& Sugarman, 1984; Stuart et al., 1990; Westen et al., 1990a, 


\section{CHAPTER 5}

1990c). As mentioned by Westen and co-workers (e.g., Stuart et al., 1990), this thinking style might be explained by the projection of aggressive impulses, higher levels of constitutional aggression and/or by expectations or schemas of the object world as being malevolent and untrustworthy possibly resulting from childhood traumas (see for the latter relation, Arntz, 1994, 2004; Ornduff, 2000) (see for a review of studies on childhood trauma in BPD, Sabo, 1997).

We investigated these issues by asking borderline patients, personality disorder (PD) controls (i.e., patients with a cluster C PD (CPD) and participants with an anti-social PD (ASPD)), and normal controls, to play two computer games and to evaluate these games as well as themselves. In this way we experimentally manipulated a non-interpersonal situation. By varying the degree of difficulty of the games, we created a frustrating, respectively rewarding situation which, we hypothesized, would activate schemas on powerlessness (of the self) and malevolence (of the non-interpersonal world), and would offer opportunity for extreme evaluations, possibly also extremely positive ones.

In summary, the main question of this study was whether BPD is characterized by dichotomous and schema-specific non-interpersonal evaluations. The hypotheses were: (1) Extreme dichotomous non-interpersonal evaluations are a specific feature of BPD; (2) Patients with BPD evaluate their non-personal environment more extremely than control subjects on dimensions related to the schema 'the world is dangerous and malevolent'; (3) Patients with BPD evaluate themselves more extremely than control subjects on dimensions related to the schemas 'I am powerless and vulnerable' and 'I am unacceptable'; (4) The extreme evaluations in BPD are unidimensional; (5) Patients with BPD show extremely negative evaluations as well as extremely positive evaluations.

\section{Method}

\section{Subjects}

Twenty-four patients with BPD, 10 patients with CPD, and 16 ASPD participants from community mental health services, mental hospitals and prisons in the Netherlands and Belgium participated. From the same regions, 25 nonpatient controls participated, who were recruited by advertisements among the general public, and by invitation to participate among staff of the collaborating institutions. All participants were screened with SCID-I and -II interviews. To be included, participants had to be between 18 and 65 years of age. Partici- 
NON-INTERPERSONAL EVALUATIONS IN BPD

Table 5.1: Demographic variables per group.

\begin{tabular}{|c|c|c|c|c|}
\hline \multirow[b]{2}{*}{$\begin{array}{l}\text { Demographic } \\
\text { variable }\end{array}$} & \multicolumn{4}{|c|}{ Group } \\
\hline & $\begin{array}{c}\text { BPD } \\
(n=24)\end{array}$ & $\begin{array}{c}\text { CPD } \\
(n=10)\end{array}$ & $\begin{array}{l}\text { ASPD } \\
(n=16)\end{array}$ & $\begin{array}{c}\mathrm{NP} \\
(\mathrm{n}=25)\end{array}$ \\
\hline Mean age $(S D)^{*}$ & $30(7.8)$ & $38(9.2)$ & $31(5.8)$ & $31(7.8)$ \\
\hline$\%$ Female $(\mathrm{N})$ & $67(16)$ & $100(10)$ & $50(8)$ & $68(17)$ \\
\hline$\%$ Married/cohabiting $(\mathrm{N})^{* * a}$ & $25(6)$ & $67(6)$ & $31(5)$ & $70(16)$ \\
\hline Education ${ }^{* b}$ & & & & \\
\hline$\%$ Lower level $(\mathrm{N})$ & $25(6)$ & $50(4)$ & $50(8)$ & $0(0)$ \\
\hline$\%$ Middle level $(N)$ & $46(11)$ & $38(3)$ & $44(7)$ & $52(12)$ \\
\hline$\%$ Higher level $(N)$ & $29(7)$ & $13(1)$ & $6(1)$ & $48(11)$ \\
\hline
\end{tabular}

pants with BPD and ASPD had to meet DSM-IV criteria for BPD respectively $A S P D$, those with CPD had to meet criteria for at least one cluster-C PD, and non-patient controls had to have no current axis-I or axis-ll disorder and not more than one disorder in complete remission. In order to create clearly discernible groups, we did not include control participants who met more than two BPD-criteria. Patients who had a bipolar or (current) psychotic disorder were also excluded, as well as mentally retarded subjects and subjects with vision problems.

Table 5.1 presents the demographic variables of the groups. The control groups differed from the BPD group in several aspects. There were differences in mean age, $F(3,72)=2.96, p<.05$. The CPD group showed a higher mean age than the BPD group, Tukey's HSD post-hoc procedure: $p<.05$. Whereas the BPD, ASPD, and nonpatient control group consisted of both women and men, the patients with CPD were all women. CPD and nonpatient participants more often lived in partnerships than the BPD and ASDP participants, $\chi^{2}(3, N=72)=16.0, p<.01$. Nonpatient controls had a relatively higher educational level than the participants with a PD, $\chi^{2}(3, N=71)=9.17, p<.05$ (two educational levels).

Regarding frequency of computer use and computer skill, we found a significant group difference in computer use. The CPD and ASPD group reported less use whereas the nonpatient group reported more use of the computer than the BPD group, $\chi^{2}(3, N=75)=11.5, p<.01$ (two levels of computer use). 


\section{CHAPTER 5}

\section{Design}

The study had a repeated pretest-posttest cross-over design. We compared the BPD group with two PD control groups (CPD and ASPD group), and a nonpatient control group. All subjects played a difficult and an easy game. In order to control for game specific effects, two different games ('tennis' and 'worm') were created which both could be played in a (fixed) difficult and easy mode. Order of the games (easy game first or difficult game first; tennis game first or worm game first) was randomly assigned to the participant and balanced over the subject groups. Subjects evaluated the game, themselves and their emotions after having played each game. They evaluated themselves and their emotions also at baseline.

\section{Apparatus}

The games were played on a personal computer with a $200 \mathrm{MHz}$ Pentium processor and a 17 inch monitor.

\section{Material}

\section{Diagnostic interviews}

DSM-IV axis I and II diagnoses were assessed with the Dutch-language versions of the SCID-I (First et al., 1997; Groenestijn et al., 1999) and SCID-II (First et al., 1994; Weertman et al., 2000). Interviewers of our centers achieved good test-retest interrater reliability with the SCID-II (Weertman et al., 2003).

\section{Computer games}

Two computer games, i.e. tennis and worm, were designed. In the tennis game subjects had to move a racket in order to return a fast or slow ball to the wall. In the worm game subjects had to direct a fast or slowly crawling worm towards targets. Scores of the subject were shown on the computer screen. In order to enlarge frustration or reward, messages with negative or positive feedback were displayed, and penalty or bonus points were given. 
NON-INTERPERSONAL EVALUATIONS IN BPD

\section{Emotional state}

Participants assessed their emotional state and evaluated themselves and the games on $100 \mathrm{~mm}$ visual analogue acales (VASs). Emotional state was measured by six VASs (i.e., happy, sad, anxious, angry, surprised, aroused) that were scored afterwards from 0 (not at all) to +100 (extremely).

\section{Self and game evaluation}

Subjects evaluated themselves on 20 trait VASs. There were three types of selfevaluation scales: (1) six specific traits related to the two BPD self-schemas (e.g., weak - strong; not accepting myself - accepting myself), (2) seven nonspecific traits with a negative and positive pole, and not related to the BPD self-schemas (e.g., boring - interesting), and ( 3 ) seven neutral traits with neutral poles and not related to the BPD self-schemas (e.g., extravert - introvert).

Participants evaluated the games on 15 trait VASs. Again there were three scale types: (1) five specific traits related to the BPD world/other-schema (e.g., bad - good), (2) five nonspecific traits with a negative and positive pole, and not related to the BPD world/other-schema (e.g., boring - exciting), and (3) five neutral traits with neutral poles and not related to the BPD world/other-schema (e.g., modern - classic).

The trait VASs were scored afterwards from -50 (pole with negative valence or randomly chosen neutral pole) to +50 (pole with positive valence or the other neutral pole). We validated the scale types by asking a separate nonpatient group $(N=10)$ to rate the VASs on relatedness to the BPD schemas and the VAS poles on emotional valence. Mean ratings showed that for both the self and game VASs, the relatedness to the BPD schemas of the specific VASs was significantly stronger than that of nonspecific and neutral VASs, and differences in emotional valence of the poles of the specific and nonspecific VASs were significantly larger than those of the neutral VASs, $t(p) s<2.87, p s<.01$.

\section{Procedure}

After having received general information about the study, all participants signed an informed consent form and reported some demographic characteristics. The screening procedure differed somewhat over the participants. Patients of one center had already been interviewed with the SCID-I and -II before the games, as part of the center's intake procedure. Other patients were interviewed be- 


\section{CHAPTER 5}

fore the games on mood and psychotic disorders with modules $A, B, C$, and $D$ of the SCID-I and on all PDs with the SCID-II. All the other participants were interviewed after having played the games, with the complete SCIDs. The participants rated their computer use (i.e., never, sometimes, regularly or often working on a computer) and computer skill (i.e., experiencing working on a computer as very hard, hard, easy, or very easy). Being then seated before the computer screen, participants filled out the emotion and self-evaluation VASs (baseline measurement), received basic instructions for the first game, and started playing the game. The experimenter shortly stayed in the room in order to check whether the participant had understood the instructions and then left the room. After five minutes (tennis game) or ten minutes (worm game), the experimenter reentered the room, stopped the game, and read aloud the score of the participant. The participant then filled out the emotion, self-evaluation and game-evaluations VASs. Having completed the VASs, the procedure from game instruction to filling out the VASs was repeated for the second game. After the second test, patients which were already interviewed with the SCIDs were thanked for their participation. The other participants were interviewed with the SCIDs during the remaining time of the first session and, if needed, one or two following sessions.

\section{Statistical analyzes}

Evaluations were investigated by looking at (1) dichotomous thinking (DT) scores, i.e., means of the absolute evaluation scores on the nonneutral and neutral VASs, or $\frac{\sum_{i=1}^{n}\left|V A S_{i}\right|}{n}$, (2) splitting scores, i.e., absolute means of the nonabsolute evaluation scores on the nonneutral VASs, or $\frac{\left|\sum_{i=1}^{m} V A S_{i}\right|}{m}$ (see Veen \& Arntz, 2000, for explanation of these calculations), and (3) negative thinking (NT) scores, i.e., the negative nontransformed scores.

Statistical tests were performed by (M)AN(C)OVAs and t-tests. Nonparametric Wilcoxon Signed Ranks tests and Mann-Whitney tests were performed when distributions were not normal according to Kolmogorov-Smirnov and Shapiro-Wilk tests or when error variances of the dependent variables were not equal across groups according to Levene's test. We investigated group differences with planned one-tailed or (if indicated) two-tailed simple contrasts (BPD vs $C P D, B P D$ vs. $A S P D$, and BPD vs. NP). We computed linear regression coefficients of the factors sex, age, and computer use with the variable under 
study as dependent varlable in order to check whether we had to apply corrections for biases caused by the group differences on these factors. Because the group differences in having a partner and educatonal level are characteristic for the groups. we did not correct the results for these variables.

\section{Results}

\section{Manipulation Check}

In order to check whether the easy and difficult games were experienced as intended, we analyzed the game scores (objective measure), game evaluations on the scales difficult - easy, frustrating - rewarding not doable - doable, and changes in emotional state from baseline and to post.game measurement (subjective measures).

\section{Game scores}

Overall, the mean game score on the difficult games $(M--35 \pi, S d=493)$ was significantly lower than the mean score on the easy games $(M=6 . x 0$,

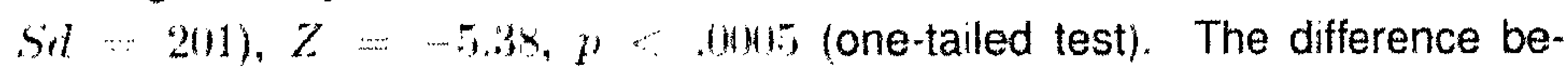
tween scores on the difficult and the easy games was similar over the groups,

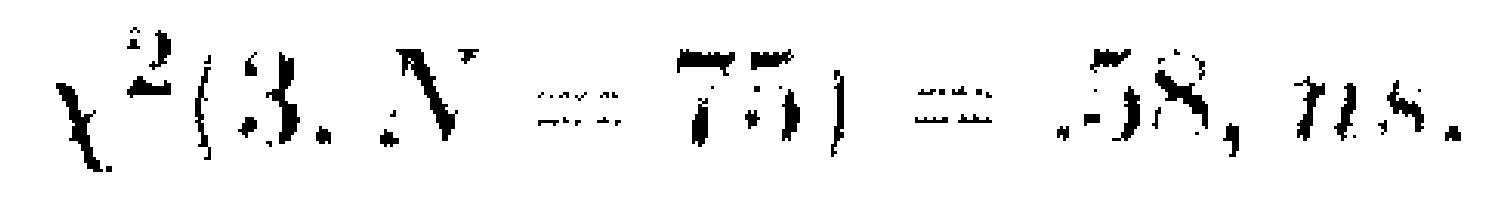

\section{Game evaluations}

The evaluations were analyzed separately for each scale. Overall, subjects evaluated the difficult games as less easy $(M=-1.90, S d=33.3), Z=$ $-2.16, p<.47$, less rewarding $(M=-1 \times .2, S i d=24.4), Z=-3.86, p<$ .0105, and less doable $(M=7 .(M), S i=32.8), Z=-6.06, p<.0015$, than the easy games (easy: $M=9.20, S d=29.3$; rewarding: $M=-6.00, S d=24.5$; doable: $M=23.7, S d=25.8$ ) (one-tailed tests). The differences between evaluations of easiness of the difficult and easy games differed over the groups, $f^{2}(3, N=75)=9.16, y<.015$. The groups evaluations of rewardfulness and doableness differed similarly from easy to difficult games over the groups, $x^{2} s$ $(3 . N=75)<3.4 ., n \mathrm{k}$. For easiness, the difference between the games was smaller for the BPD group than for the ASPD group, $t(3 x)=2.29, p<.15$, and in further analyzes it appeared that of the four groups only the ASPD group evaluated the easy game as significantly easier $(M \ldots 9.5(, . S d=30.3)$ than 


\section{CHAPTER 5}

the difficult game $(M=-22.6, S d=20.8), Z=-2.64, p<.005$ (one-tailed test).

\section{Emotional state}

Only emotions with clear valences were analyzed, i.e., happiness,sadness, fear, and anger. After the difficult game, the subjects reported less happiness ( $M=$ $39.5, S d=23.3), Z=-4.35, p<.0005$, and more anger $(M=20.3, S d=$ 24.7), $Z=-3.18, p<.0005$, than at baseline (happiness: $M=55.4, S d=$ 25.3; anger: $M=12.0, S d=17.3$ ) (one-tailed tests). Sadness (baseline: $M=24.9, S d=26.0$; difficult game: $M=23.5, S d=26.1$ ) and fear (baseline: $M=24.0, S d=28.6$; difficult game: $M=21.1, S d=26.5$ ) did not significantly increase from baseline to difficult game, $|Z| \mathrm{s}<.88$, ns (one-tailed tests). After the easy game, the subjects reported less sadness (baseline: $M=24.9, S d=$ 26.0; easy game: $M=19.7, S d=23.4), Z=-2.24, p<.05$, but not more happiness, $Z=-2.78, n s$, nor less anger or fear, $|Z| \mathrm{s}<.62$, $n s$ (one-tailed tests). In fact, there was a decrease of reported happiness after the easy game, (easy game: $M=46.0, S d=23.1$ ), $Z=-2.78, p<.005$ (two-tailed test).

Most emotion changes from baseline to post-game measurement were similar over the groups, $\chi^{2} \mathrm{~s}(3, N=75)<7.07, n s$. Only the decrease in fear from baseline to easy game differed significantly over the groups, $\chi^{2}(3, N=$ $75)=10.4, p<.05$. The BPD group showed a significant decrease of fear after the easy game (baseline: $M=36.7, S d=29.8$; easy game: $M=30.5$, $S d=27.6), Z=-1.77, p<.05$, whereas the others did not (one-tailed tests).

\section{Manipulation check summarized}

Playing the difficult game resulted in low game scores, was found to be not very rewarding and doable, decreased happiness, and increased anger. Compared to the difficult game, the easy game resulted in high game scores and was evaluated as rewarding and doable. Although playing the easy game reduced happiness, it also reduced sadness in all groups and fear in the target BPD group. We conclude that we successfully manipulated non-interpersonal frustration and reward. 


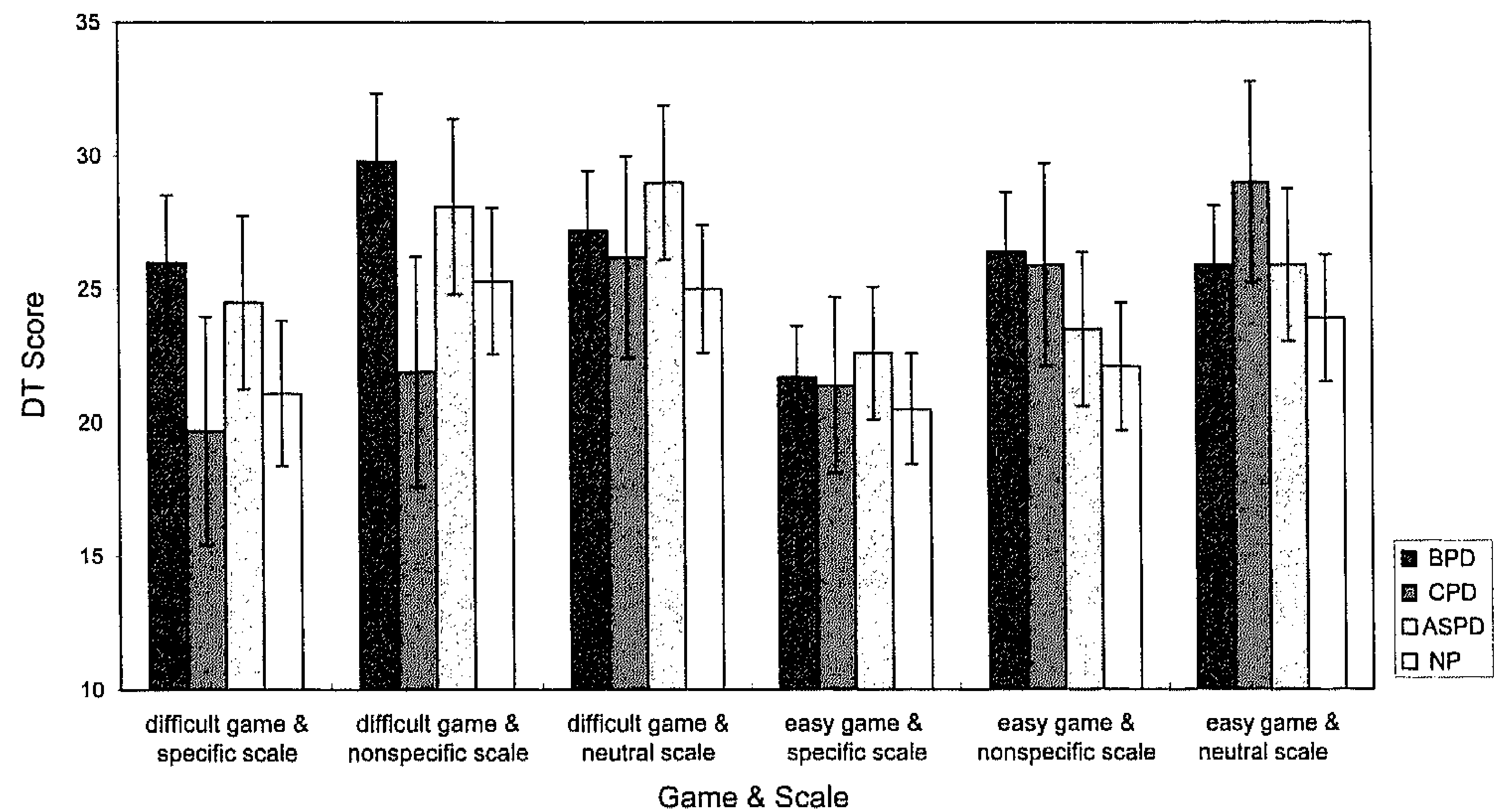

Figure 5.1: DT scores (with standard errors) per subject group, game, and scale type.

\section{Game evaluations}

\section{DT scores}

DT scores are presented in Figure 5.1. Contrasts on the nonneutral scales were in the expected direction with exception of the contrast BPD vs. ASPD for DT scores of the easy game on the specific scale. The contrasts however were mostly not significant, as is shown in Table 5.2. For the difficult game, only the contrast BPD vs. CPD for evaluations on the nonspecific scales reached significance. For evaluations on the specific scales this contrast (BPD vs. CPD) was a trend. For the easy game, all contrast were nonsignificant.

\section{Splitting scores}

Figure 5.2 illustrates the splitting scores. Contrasts on the nonneutral scales were again in the expected direction with exception of the contrast BPD vs. ASPD for evaluations of the easy game on the specific scales. Just as the DT scores however, the splitting scores also not showed large group effects (see Table 5.2). For the difficult game, the contrast BPD vs. NP for evaluations on the specific scales was the only significant contrast. 
CHAPTER 5

Table 5.2: Test results ${ }^{1}$ of the group contrasts for the DT, Splitting, and NT St the game evaluations.

\begin{tabular}{|c|c|c|c|}
\hline \multirow[b]{2}{*}{ Contrast } & \multicolumn{3}{|c|}{ Game Evaluation Scores } \\
\hline & DT & Splitting & NT \\
\hline \multicolumn{4}{|c|}{ Difficult game on specific scales } \\
\hline BPD vs. CPD & $1.37^{\dagger}$ & $1.43^{\dagger}$ & 1.00 \\
\hline BPD vs. ASPD & 0.32 & 0.45 & $3.61^{* * *}$ \\
\hline BPD vs. NP & $1.46^{\dagger}$ & $210^{*}$ & $1.70^{*}$ \\
\hline \multicolumn{4}{|c|}{ Difficult game on nonspecific scales } \\
\hline BPD vs. CPD & $1.77^{*}$ & $86.0^{\dagger}$ & $1.48^{\dagger}$ \\
\hline BPD vs. ASPD & 0.49 & 0.99 & $2.48^{* *}$ \\
\hline BPD vs. NP & 1.09 & $223^{\dagger}$ & $1.70^{*}$ \\
\hline \multicolumn{4}{|c|}{ Difficult game on neutral scales } \\
\hline BPD vs. CPD & 0.18 & & \\
\hline BPD vs. ASPD & -0.53 & & \\
\hline BPD vs. NP & 0.58 & & \\
\hline \multicolumn{4}{|c|}{ Easy game on specific scales } \\
\hline BPD vs. CPD & 0.28 & 107 & -0.08 \\
\hline BPD vs. ASPD & 176 & -1.29 & $1.83^{*}$ \\
\hline BPD vs. NP & 240 & 284 & 271 \\
\hline \multicolumn{4}{|c|}{ Easy game on nonspecific scales } \\
\hline BPD vs. CPD & 0.53 & 119 & 0.55 \\
\hline BPD vs. ASPD & 0.78 & 159 & $1.27^{\dagger}$ \\
\hline BPD vs. NP & $1.48^{\dagger}$ & 291 & 0.66 \\
\hline \multicolumn{4}{|c|}{ Easy game on neutral scales } \\
\hline BPD vs. CPD & -0.08 & & \\
\hline BPD vs. ASPD & 0.18 & & \\
\hline BPD vs. NP & 0.84 & & \\
\hline
\end{tabular}




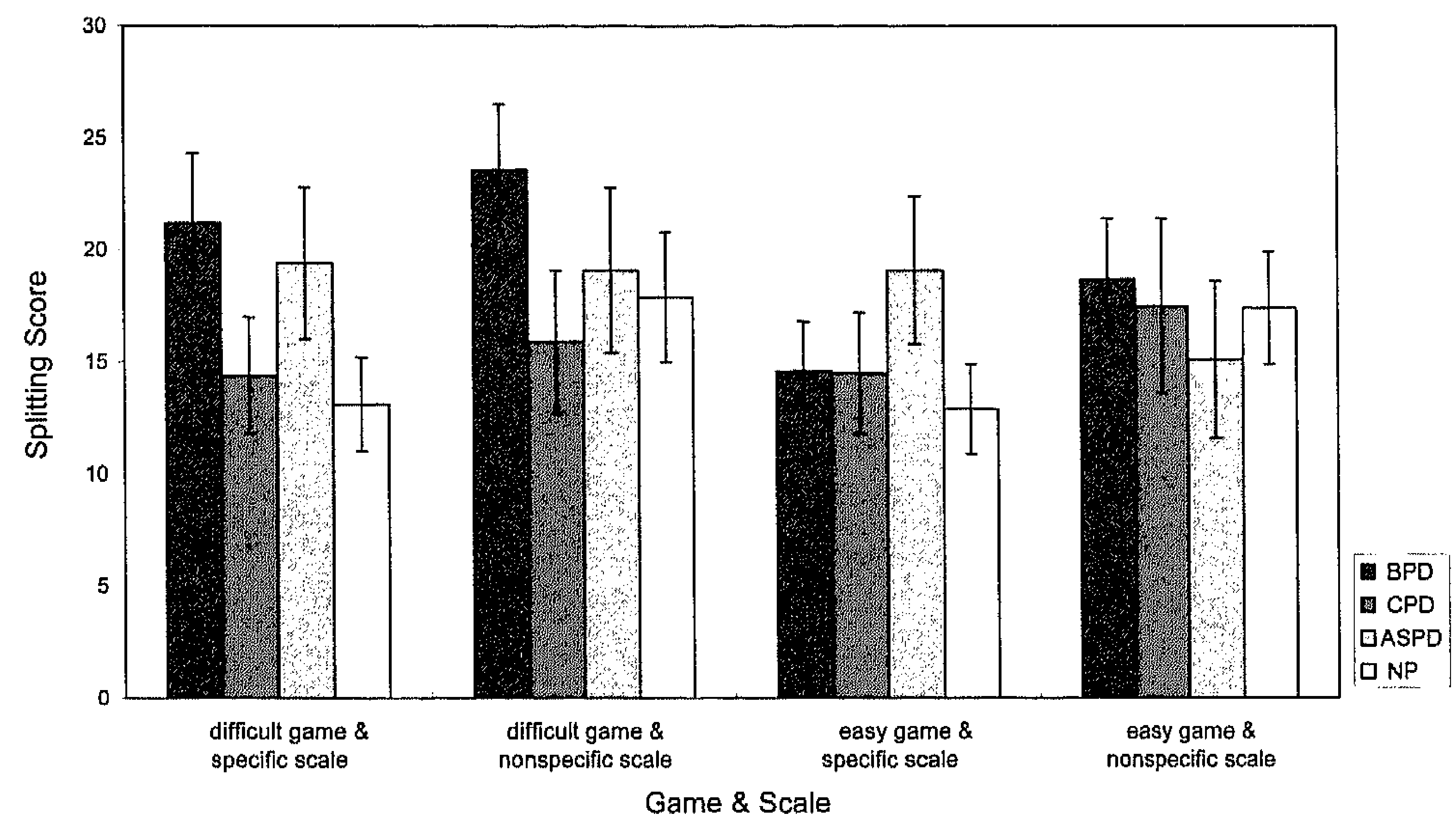

Figure 5.2: Splitting scores (with standard errors) per subject group, game, and scale type.

\section{NT scores}

Mean NT scores of the game evaluations on the non-neutral scales are presented in Figure 5.3. Patients with BPD evaluated the difficult game more negatively than most other groups. Unlike the DT and splitting scores, contrasts with BPD for the NT scores were, at least for the difficult game, mostly significant. Only the contrasts BPD vs. CPD failed to reach significance, although we did find a trend for this contrast for evaluations on the nonspecific scales.

Because the ASPD group evaluated the games mostly more positively instead of more negatively, we also looked at the contrasts ASPD vs. CPD, and ASPD vs. NP. For evaluations of the difficult game, the contrasts ASPD vs. CPD for the specific scale, $t(32)=-1.96, p<.05$, and ASPD vs. NP for the specific scale, $t(47)=-2.13, p<.05$, were significant. For the easy game, these contrasts were nonsignificant.

DT, splitting, and NT scores were not corrected for group differences in age, sex, and computer use because regression coefficients for these scores were nonsignificant in all Game by Scale conditions. 


\section{CHAPTER 5}

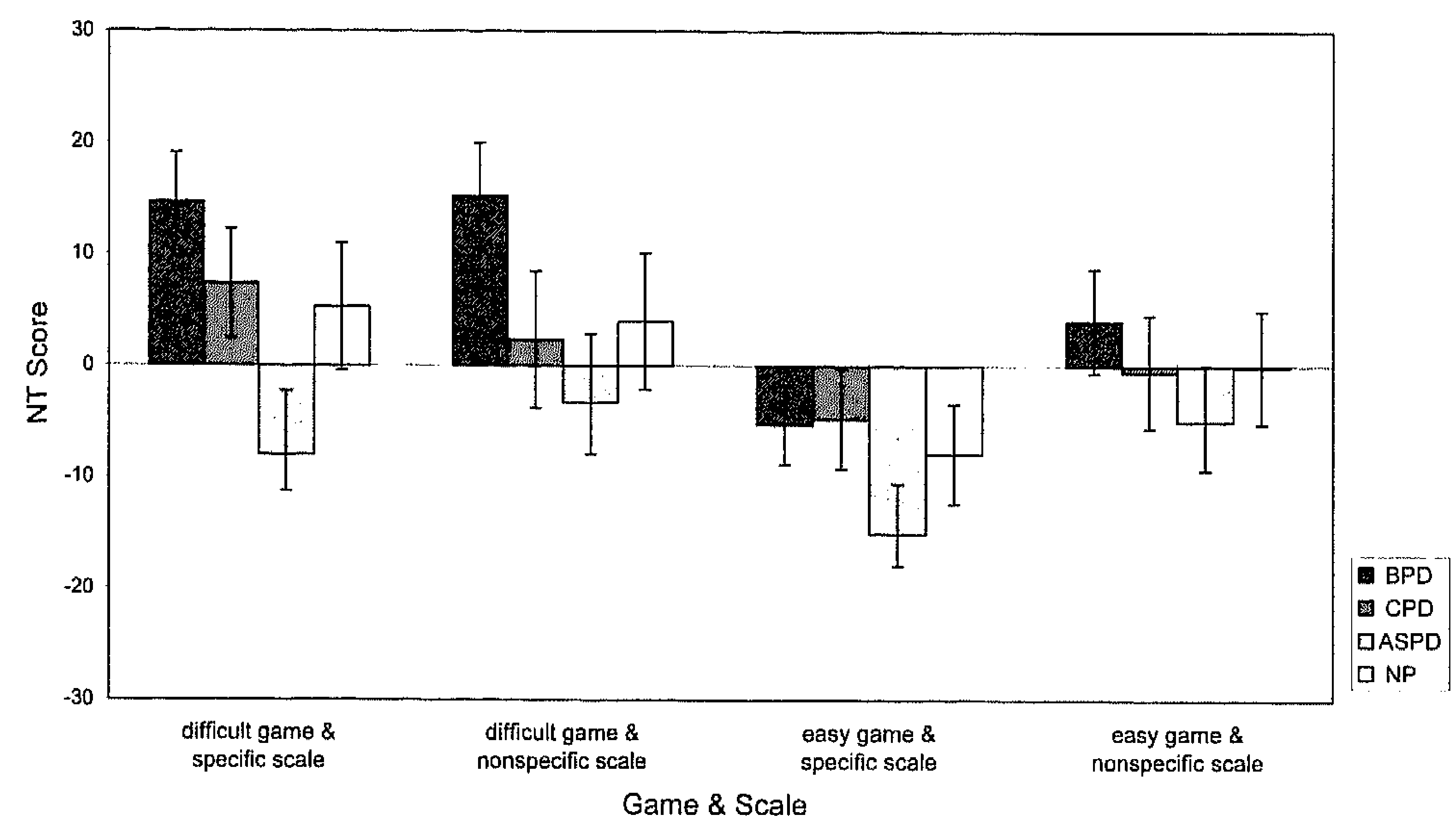

Figure 5.3: NT scores (with standard errors) per subject group, game, and scale type.

\section{Self-evaluations}

\section{Baseline}

At baseline, patients with BPD showed lower DT scores for self-evaluations than the ASPD group on the nonspecific scales (BPD: $M=23.8, S D=9.26$; ASPD: $M=28.3, S D=10.8), t(38)=2.29, p<.05$, and than the NP group on the specific scales (BPD: $M=26.4, S D=9.90$; NP: $M=34.7$, $S D=11.2), t(47)=2.81, p<.01$. DT scores were not corrected for group differences in sex, age, and computer use because regression coefficients were nonsignificant for all the scales.

Patients with BPD showed also low splitting scores for self-evaluations at baseline. Two-tailed tested contrasts that reached significance were the contrast with the ASPD group for the nonspecific scale (BPD: $M=13.4, S D=$ 10.4; ASPD: $M=27.0, S D=10.0), U=68.0, p<.0005$, and with the NP group for both scales: specific (BPD: $M=13.0, S D=9.13$; NP: $M=19.4$, $S D=9.19), U=191, p<.05$, and nonspecific scales (NP: $M=24.4$, $S D=13.1), t(47)=3.47, p<0.005$ (two-tailed tests). The regression coefficient of the factor computer use was significant for splitting scores on the specific scales $(\beta=.28), t=2.45, p<.05$. All other regression coefficients were 
nonsignificant. The analyzes were corrected by adding computer use as covariate to the ANOVA. For the Mann-Whitney's test we recalculated the scores for each subject for one common level of computer use, using the regression coefficient. The corrections did not change the results.

NT scores of the self-evaluations at baseline were also low for patients with BPD. The BPD group evaluated themselves less positively than the ASPD group on the specific scales (BPD: $M=-6.07, S D=14.9$; ASPD: $M=15.9, S D=$ 9.78), $t(38)=5.80, p<.0005$, and the nonspecific scales (BPD: $M=5.07$, $S D=16.4$; ASPD: $M=27.0, S D=10.0$ ), $U=48.0, p<.0005$ (twotailed tests). The BPD group evaluated themselves also less positively than the NP group, again on both scales, viz. the specific scales (NP: $M=19.4$, $S D=9.19$ ), $U=46.5, p<.0005$, and the nonspecific scales (NP: $M=24.3$, $S D=13.1), t(47)=4.87, p<.0005$ (two-tailed tests). Mean evaluation scores were not corrected for group differences in sex, age and computer use because regression coefficients were nonsignificant for all the scales.

\section{Effect of games on self-evaluations}

The effects of the games on the self-evaluations were analyzed with ANCOVAs with scores at baseline as covariates and the DT, splitting or NT scores as dependent variables, or with Mann-Whitney tests of the change in scores (postgame score - score at baseline).

The BPD group showed in almost all conditions lower instead of higher DT and splitting scores than the other groups. No contrast reached significance. Regression coefficients of sex, age and computer use were nonsignificant for the DT and splitting scores in all Game by Scale conditions.

For the NT self-evaluation scores after the difficult game, we found that patients with BPD evaluated themselves significantly more negatively than those with ASPD on the specific scales (BPD: $M=6.63, S E=2.04$; ASPD: $M=$ $11.8, S E=2.33), t(38)=1.89, p<.05$. No significant contrasts were found for the easy game.

Again, we also investigated indications for a positivity bias in ASPD by looking at the contrasts with this group. The ASPD group showed significantly more positive self-evaluations than the NP group on the specific scales after both the difficult game (changes from baseline to difficult game: ASPD: $M=1.15$, $S d=9.08$; NP: $M=-1.99, S d=7.00), U=119, p<.05$, and the easy game (changes from baseline to easy game: ASPD: $M=2.76, S d=4.51$; NP: $M=-.72, S d=5.59), U=110, p<.05$. All other contrasts were 


\section{CHAPTER 5}

nonsignificant.

Regression analyzes yielded significant coefficients for the factor sex on the nontransformed self-evaluation score on the specific scales after the difficult game ( $\beta=-.29$, i.e., women show lower scores), $t=-2.53, p<.05$, and for the factor computer use on the same scales after the easy game $(\beta=.25$, i.e., subjects high on computer use show higher scores), $t=-2.16, p<.05$. All other regression coefficients were nonsignificant. We corrected for possible biases of group differences in sex on the analyzes for the specific scales after the difficult game by redoing these analyzes with the female subjects only. For the female subjects, the contrasts BPD vs. ASPD patients and ASPD vs. NP group for nontransformed self-evaluations on the specific scales of the difficult game disappeared. Biases of group differences in computer use on the analyzes for the specific scales after the easy game were corrected for by adding computer use as an additional covariate, or (for non-parametric analyzes) by recalculating the scores for each subject for one common level of computer use, using the regression coefficient. After correction, a borderline significant contrast for this condition appeared between the BPD and ASPD group (BPD: $M=10.4$, $S E=2.50 ;$ ASPD: $M=11.2, S E=3.56), t(38)=1.78, p=.05$.

\section{Multidimensional evaluations versus one-dimensional evaluations}

In order to investigate whether the evaluations were multidimensional or unidimensional, we compared the DT scores and splitting scores for the games and self-evaluations. The BPD group showed significantly higher DT scores than splitting scores for both game and self-evaluations in all conditions, $.0005<p s$ $<.03$, indicating that DT of these patients was multidimensional instead of unidimensional.

\section{Discussion}

The hypothesis that patients with BPD make more extreme dichotomous evaluations than others in non-interpersonal situations was not convincingly supported. The patients with BPD did show somewhat more extreme evaluations of their nonpersonal environment, i.e., the computer games, when they were frustrated, compared with normal controls or patients with CPD, both on measures for multidimensional DT and unidimensional DT or splitting. These effects were however not consequently significant and the evaluations of the patients 
with BPD were not very extreme (i.e., about half-way the range). Patients with BPD did not at all evaluate themselves more extremely than the controls in these non-interpersonal situations. They were as negative about themselves as they were prior to the experiment.

The weak effects might indicate that non-interpersonal DT in BPD is not a major cause for dysfunctional behavior of patients with BPD in non-interpersonal situations. Methodological weaknesses however may also have reduced the effects. Although emotional state of the participants was affected by the games, the emotional induction may have been too weak. Further, if DT is dependent on schema activation as has been found before (Veen \& Arntz, 2000), these schemas might not have been sufficiently activated by the tennis and worm games that were not explicitly unreliable but rather fair and straightforward. Not finding extreme self-evaluations might be attributed to the unfamiliar computer game that possibly formed a better object for directing negative (or positive) feelings at than the subject self. A familiar task, such as a household task, might activate the vulnerability self-schema better.

The data do suggest that negative thinking discriminates BPD from other groups, at least in negative emotional non-interpersonal situations. In the negative situation, patients with BPD showed significantly more negative thinking than normal controls and ASPD participants, and almost significantly more than CPD participants. This is in accordance with the studies of Westen and colleagues (e.g., Stuart et al., 1990). Finding negative thinking in a noninterpersonal context might mean that negative interpersonal thinking resulting from interpersonal childhood trauma's has been generalized to non-interpersonal situations. It might also mean that these traumas are not necessary precursors, but that higher levels of constitutional aggression, form a sufficient condition for this cognitive style.

The present study did not find indications for the hypothesized schema specificity of non-interpersonal evaluations of borderline patients. Patients with BPD showed higher scores for DT, splitting and negative thinking on dimensions related as well as unrelated to the schema that the world is dangerous and malevolent. For self-evaluations no differences were found between patients with BPD and the other groups on any dimension and thus also not for dimensions related to BPD schemas on a powerless and unacceptable self. Cognitive models in which DT is closely tied to dysfunctional schema activation (Layden et al., 1993) were therefore not supported for non-interpersonal evaluations. This however might also be an artifact of finding moderate levels of DT 


\section{CHAPTER 5}

in BPD.

Evaluations of the borderline patients appeared not unidimensional (unipolar) but multidimensional (multipolar). Patents with BPD judged some aspects of their environment and themselves as negative and other aspects as positive, and did not show all-bad or all-good evaluations. This does not support the hypothesis that extreme evaluations of patients with BPD in non interpersonal situations are expressions of the defense mechanism splitting. Again, however this might be an artifact of the weak emotion induction. or of the moderate levels of extreme thinking in the BPD group.

A final remark concerns the unforeseen similarities and contrasts that were found between the BPD and ASPD group. BPD and ASPD participants showed rather similar levels of extreme thinking, but whereas the first mostly showed negative thinking, the latter showed a positivity bias. Because ASPD participants were mostly prisoners whereas BPD participants were all patients, it is possible that factors related to being imprisoned instead of diagnosis account for the positivity bias. If this finding can be replicated in a sample of only patients with ASPD it adds to other common and contrasting features of BPD and ASPD (see for example. Coccaro, 1989; Herpertz et al., 2001b; Lobbestael, Arntz, \& Sieswerda, 2005; Siever \& Davis, 1991) and deserves further study.

Summarizing, the results of the present study indicate that in non-interpersonal emotionally negative situations BPD patients appear to be characterized by a negativistic thinking style, more than by DT or splitting that seem to be unique for interpersonal situations. 


\title{
Chapter 6
}

\section{Change of Extreme and Negative Interpersonal Evaluations in Borderline Patients During Psychotherapy ${ }^{1}$}

\begin{abstract}
Cognitive and psychodynamic models hypothesize that extreme evaluations are central in borderline personality disorder (BPD). This study investigated whether extreme and negative interpersonal evaluations of patients with BPD decrease during psychotherapy, whether these decreases are positively related to a reduction of BPD severity, and what type of evaluations are most indicative of BPD pathology. Patients with BPD were asked to evaluate characters from film clips with negative, positive, or neutral roles, on specific, nonspecific, and neutral visual analogue scales, at start $(\mathrm{N}=78)$, during $(\mathrm{N}=64)$, and end $(\mathrm{N}=46)$ of a long-term change oriented psychotherapy. Results supported the hypotheses that extreme evaluations characterize BPD, decrease during psychotherapy, and that reduction of these evaluations is correlated with decrease in BPD manifestations, particularly during the first half of treatment. All good / all bad evaluations were not prominent. Decrease of negative evaluations was not related to change in BPD level and may be rather stable.
\end{abstract}

\footnotetext{
${ }^{1}$ The content of this chapter has been submitted for publication as Sieswerda, S. \& Arntz, A., Change of extreme and negative interpersonal evaluations of borderline patients during psychotherapy.
} 


\section{CHAPTER 6}

\section{Introduction}

Both cognitive and psychodynamic models assume that extreme interpersonal evaluations play a central role in the extreme and unstable affects and behaviors characterizing borderline personality disorder (BPD; American Psychiatric Association, 1994). Cognitive models of BPD assume that borderline patients' affects and behaviors are destabilized by a combination of negative maladaptive schemas and dichotomous thinking, i.e., making 'black-and-white' or 'allor-none' evaluations (Arntz, 2004; Pretzer, 1990; Layden et al., 1993). Psychodynamic theories hypothesize that the instability and extremes of patients with BPD are caused by an immature personality structure in which good and bad representations of others are passively or actively split off (e.g., Kernberg, 1996). In line with these models, cognitive therapies (e.g., Pretzer, 1990; Young et al., 2003) challenge maladaptive negative schemas and dichotomous thinking, and psychodynamic therapies (e.g., Clarkin et al., 1999) aim integrating split object representations.

Empirical studies have provided ample support for negative schemas in BPD. Cross-sectional studies have demonstrated that borderline patients show more extreme negative evaluations of stimuli than other patients and psychologically healthy controls. These stimuli varied from interpersonal to non-personal stimuli, i.e., from persons at pictures (Ackerman et al., 1999; Nigg et al., 1992; Segal et al., 1992; Stuart et al., 1990; Westen et al., 1990a, 1990b, 1990c, 1990d), characters in film clips (chapter 4 of this thesis; Arntz \& Veen, 2001), the patients' own mothers and themselves (Benjamin \& Wonderlich, 1994), to computer games (chapter 5 of this thesis) and words (Kurtz \& Morey, 1998). BPD thus appears to be characterized by a negativistic evaluative style, even extending beyond the interpersonal context.

Dichotomous thinking and/or splitting, i.e., making both extreme negative and extreme positive evaluations, have less consistently been found to characterize borderline patients. Two studies investigating evaluations of film characters (Veen \& Arntz, 2000) and of telephone counselors (Ten Haaf \& Arntz, 2009) have shown that borderline patients give more extreme (negative and/or positive) evaluations than other patients and healthy persons. These studies however did not separate negative and positive evaluations (Veen \& Arntz, 2000 ), or separated these but found significant effects for more negative evaluations only (Ten Haaf \& Arntz, 2009). Furthermore, the spontaneous evaluations of the borderline patients examined in the first study (Arntz \& Veen, 2001), and 
evaluations of another borderline patient sample and a wider range of film character stimuli assessed with a similar paradigm (see chapter 4) appeared to be only more negative instead of more negative and positive.

Extreme evaluations of borderline patients were furthermore always multidimensional, i.e., mixed negative and positive (Veen \& Arntz, 2000; Ten Haaf \& Arntz, 2009), and not unidimensional, i.e., consisting of only negative or only positive evaluations. These findings fundamentally contradict the psychodynamic model of BPD because this model assumes a split of 'all good' and 'all bad' object representations. The cognitive model does not exclude multidimensional dichotomous thinking and can explain abrupt shifts and instability in BPD in other ways than by shifts between 'all good' and 'all bad' evaluations. For example, shifts between polarized dichotomous evaluations along dimensions related to certain themes, or approach-avoidance conflicts between concurrent contradictory extreme evaluations.

The present study further investigated extreme thinking (ET) and negative thinking (NT) in BPD by examining patients undergoing long-term change oriented psychotherapy. If ET and NT are major factors in BPD, these characteristics should be positively related to level of BPD severity and should reduce during successful treatment. A longitudinal design has certain advantages relative to the cross-sectional designs applied in previous studies. By comparing ET within-subjects instead of between-subjects influences of other individual differences on evaluations than having BPD, for example having an emotional temperament, can be excluded. Changes during psychotherapy further show what is feasible within a patient and within a realistic time frame, instead of what would be theoretically desirable, but not attainable.

Interpersonal evaluations were experimentally assessed by showing patients film clips and asking them to judge the characters in these clips on several visual analogue scales (VASs). This method has been successfully applied in two other studies (chapter 4 of this thesis; Veen \& Arntz, 2000). In order to study changes during treatment evaluations were assessed at start, halfway, and at end of treatment. Because some authors (e.g., Layden et al., 1993) assume that borderline patients only think extremely and negatively in situations activating specific schemas and/or negative emotional childhood memories, we differentiated film character roles and scales in several types, maximizing chances of finding ET or NT. This also made it possible to explore the kind of interpersonal ET or NT most indicative of BPD pathology.

In sum, the primary goal of this study was to investigate the centrality of 


\section{CHAPTER 6}

extreme and NT in BPD, in particular change in extreme or negative interpersonal evaluations in BPD through (psychodynamic or cognitive) psychotherapy. We hypothesized: (1) extreme and negative interpersonal evaluations of patients with BPD decrease during psychotherapy, and (2) these decreases are positively related to a reduction of BPD severity. We further explored (3) the multi- or unidimensionality of the extreme evaluations, (4) interpersonal stimulus types and scales inducing evaluations most indicative of change in BPD pathology, and (5) the broad time line of evaluation changes related to change in BPD pathology.

\section{Method}

\section{Participants}

The participants of the present study had sought outpatient treatment for BPD at one of four community mental health centers in the Netherlands. They were willing to participate in a randomized clinical trial, comparing Schema Focused Therapy (SFT) and Transference Focused Psychotherapy (TFP) (see, GiesenBloo et al., 2006b) in return for free treatment. Participants were screened with Dutch versions of the SCID-I (First et al., 1997; Groenestijn et al., 1999) and SCID-II interviews (Spitzer et al., 1995; Weertman et al., 2000) for DSM-IV axis I and II disorders (American Psychiatric Association, 1994), and with the BPD Severity Index (BPDSI: Arntz et al., 2003; Giesen-Bloo et al., 2006c). Participants suspect of a dissociative disorder were additionally screened with the SCID-D (Steinberg, 1993). In order to be included, participants had to fulfill the DSM-IV criteria for BPD, and have a BPDSI score $\geq 20$, indicating a moderate to severe form of BPD. Persons with a (current) psycho-organic disorder, attention-deficit/hyperactivity disorder, severe addiction needing clinical detoxification, bipolar disorder, psychotic disorder, dissociative identity disorder, or antisocial personality disorder were not admitted to the study.

The first, second, and third assessment ( $T 1, T 2, T 3$ ) took place respectively at three, 18, and 36 months after start of treatment and included respectively 78 , 64, and 46 patients. Fourteen participants dropped out of treatment between T1 and T2. Another 10 patients dropped out of treatment between T2 and T3, and eight others were not tested at T3 for other reasons (e.g., refusal of being tested, emigration).

Table 6.1 presents the demographic variables and diagnostic characteristics 
assessed at start of treatment of the BPD groups tested at $T 1$ and $T 2$, and at T2 and T3. Demographic and diagnostic variables at start of treatment did not differ between the patients that were tested at $\mathrm{T} 1$ and $\mathrm{T} 2$, and at $\mathrm{T} 2$ and $\mathrm{T} 3$.

Table 6.1: Demographic and diagnostic variables at start of treatment of patients tested at $T 1$ and $T 2$, and at $T 2$ and $T 3$ !

\begin{tabular}{|c|c|c|}
\hline & \multicolumn{2}{|c|}{ Group } \\
\hline \multirow[t]{2}{*}{ Variable } & $T 1 \& T_{2}$ & $T 2 \& T 3$ \\
\hline & $(N=64)$ & $(N=46)$ \\
\hline M Age & $31(8.5)$ & $30(8.3)$ \\
\hline$\%$ Female & $92(59)$ & $89(41)$ \\
\hline$\%$ Education high - middle level ${ }^{2}$ & $45(29)$ & $41(19)$ \\
\hline$\%$ Employed/studying & $36(22)$ & $34(15)$ \\
\hline$\%$ Married/cohabiting & $33(21)$ & $33(15)$ \\
\hline \multicolumn{3}{|l|}{ SCID-1 } \\
\hline$\% \operatorname{mood}$ & $58(37)$ & $52(24)$ \\
\hline$\%$ anxiety & $78(50)$ & $80(37)$ \\
\hline$\%$ substance & $23(15)$ & $20(9)$ \\
\hline$\%$ somatoform & $16(10)$ & $15(7)$ \\
\hline$\%$ eating & $16(10)$ & $13(6)$ \\
\hline M\# disorders & $2.8(1.6)$ & $2.9(1.6)$ \\
\hline \multicolumn{3}{|l|}{ SCID-II } \\
\hline$\%$ cluster A PD & $18(12)$ & $22(10)$ \\
\hline$\%$ cluster BPD, not BPD & $8(5)$ & $9(4)$ \\
\hline$\%$ cluster C PD & $47(30)$ & $50(23)$ \\
\hline M \# disorders & $1.9(0.9)$ & $2.0(1.0)$ \\
\hline M BPDSI & $33(8.0)$ & $34(8.4)$ \\
\hline M BPD Checklist & $120(27)$ & $120(26)$ \\
\hline \multicolumn{3}{|c|}{$\begin{array}{l}\text { Standard deviations or frequencies are between parentheses. }{ }^{2} \text { From university education to } \\
\text { vocational or technical training } 21 \times \text { years of age. Others had received vocational or technical } \\
\text { training }<1 \% \text { years of age to no education. }{ }^{3} \text { Others were unemployed or on long-term sick } \\
\text { leave. }{ }^{2} \text { Three and two missing values for } T 1 \& T 2 \text {, and } T 2 \& T 3 \text {, respectively. }{ }^{5} \text { One missing } \\
\text { value for both groups. }\end{array}$} \\
\hline
\end{tabular}




\section{CHAPTER 6}

\section{Design}

The study had a prospective longitudinal design. Changes in BPD severity were related to changes in multidimensional ET (mET), unidimensional ET (UET), and NT (NT) scores, during the first and second half of treatment, across conditions of Role Type (negative, positive, neutral) and Scale (mET scores: specific, nonspecific, neutral; $\mathrm{UET}$ and NT scores: specific, nonspecific).

\section{Treatment}

The patients were treated with SFT $(59 \%)$ or TFP $(41 \%)$. SFT is a cognitive behavioral psychotherapy with experiential elements, based on the schema-mode model of BPD of Young et al. (2003). TFP is a psychodynamic psychotherapy focusing on the here and now, based on Kernberg's model of borderline personality organization (Clarkin et al., 1999). The treatments were given for three years in biweekly sessions by psychotherapist experienced in treating BPD and participating in peer-supervision and supervision meetings (see also, GiesenBloo et al., 2006b).

\section{Material}

\section{Diagnostics}

Dutch-language versions of the SCID-I (First et al., 1997; Groenestijn et al., 1999), SCID-D (Steinberg, 1993), and SCID-II (First et al., 1994; Weertman et al., 2000) were used to assess DSM-IV diagnoses. The SCID-II has a good test-retest interrater reliability (Weertman et al., 2003). Current severity of BPD was measured with the BPD Checklist (Arntz \& Dreessen, 1992). The BPD Checklist is a self-report questionnaire consisting of 475 -point Likert scale items on which participants can indicate to what degree they have been troubled by a wide range of DSM-IV BPD symptoms during the past month (range: 47235). The BPD Checklist has a very good internal consistency (Cronbach's $\alpha>.92$ ), construct, concurrent, and discriminant validity, and has been proved sensitive to change during treatment (Arntz et al., 2003; Giesen-Bloo et al., 2006a). 


\section{Characters}

At each assessment, participants evaluated 9 to 11 characters presented in four film clips. Each film clip was five to ten minutes long and could be understood without prior knowledge. The characters had a negative, positive, or neutral role. Negative and positive roles were all involved in an emotional interaction within the primary support group (such as a partnership, a parent-child interaction, or an intimate friendship). There were three types of negative roles: victim role (e.g., a child abused by a parent), perpetrator role (e.g., a parent abusing her or his child), and ambiguous perpetrator role (e.g., a woman or a man both offending their partner and being offended by their partner). There were two types of positive roles: helper role (e.g., a parent figure helping an abused child), and intimate role (e.g., a parent figure and child, or two partners in a positive intimate interaction in a positive context). Characters with neutral roles were involved in relatively non-emotional interactions (e.g., a non-emotional interaction of a couple on vacation). Table 6.2 provides descriptions of the characters and film clips.

To check whether the characters accurately represented the role types, seven independent judges ascribed role types to the characters, using definitions of the role types and a free-response option. Seven of the 30 characters were ascribed to the correct role type by less than five of the seven judges. We corrected for these characters, by running secondary analyzes of corrected scores, calculated without the evaluations of these characters.

\section{Emotional state}

Participants rated the degree in which they felt happy, sad, anxious, angry and aroused on five $100 \mathrm{~mm}$ VASs. Ratings were scored from 0 (not at all) to +100 (extremely). The first four emotion ratings were converted into one composite positive emotion score by averaging the rating of the positive emotion (happy) and the mirrored ratings of the three negative emotions (sad, anxious, angry).

\section{Character evaluations}

Participants evaluated each character on specific, nonspecific and neutral trait VASs represented by: (1) 10 traits related to the BPD schemas (e.g., powerless - powerful, bad - good, rejecting - accepting), (2) five traits unrelated to the BPD schemas, with a negative and positive pole (e.g., stingy - generous), and (3) 


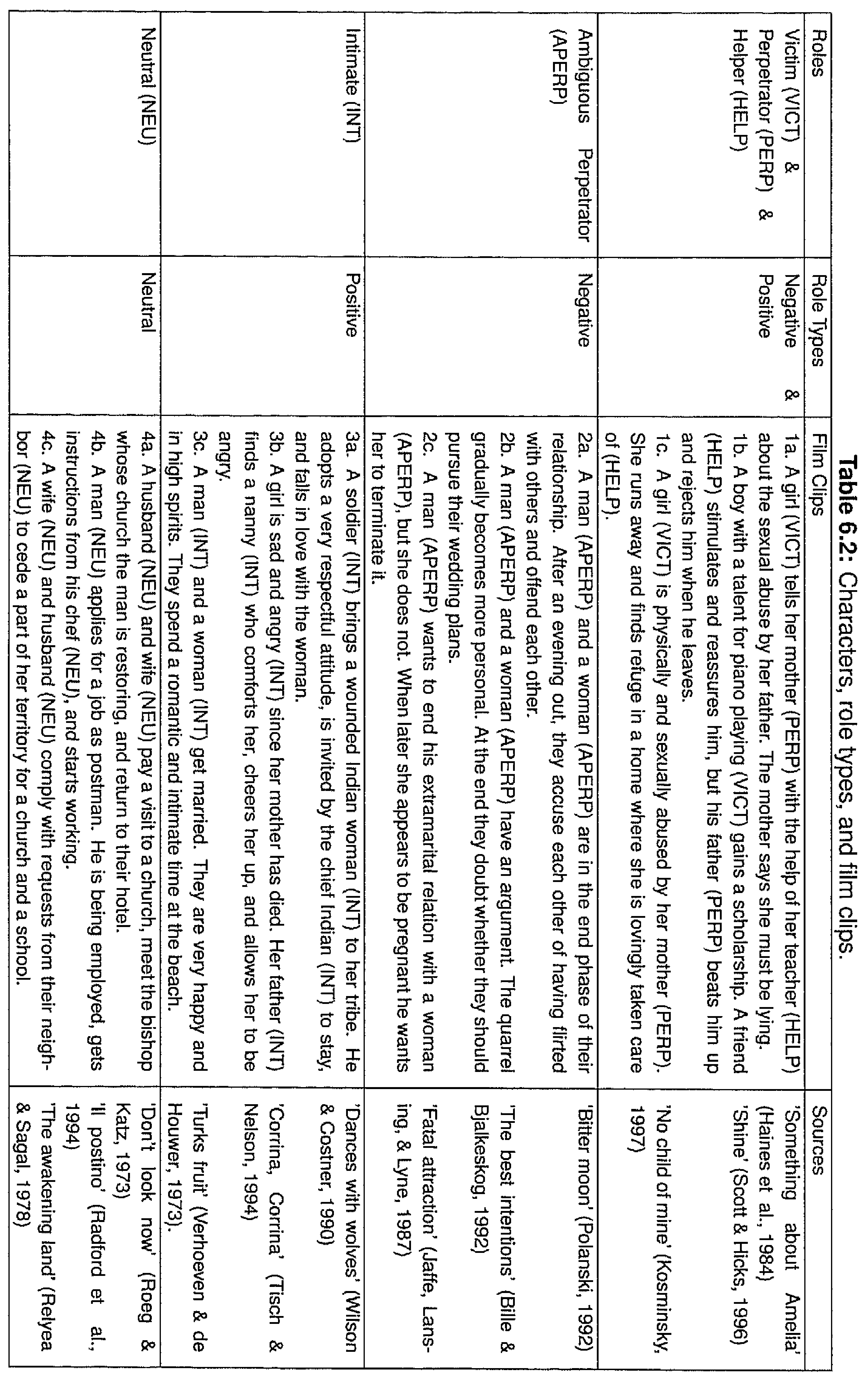


five traits not related to the BPD schemas. with neutral poles (e.g. persistent - flexible). Evaluations were scored from -50 (pole with negative valence or randomly chosen neutral pole) to +50 (pole with positive valence or the other neutral pole).

Evaluations of the characters were converted to $11 / \mathrm{mET}$ scores, i.e., means of the absolute evaluation scores on the nonneutral and neutral VASs. or

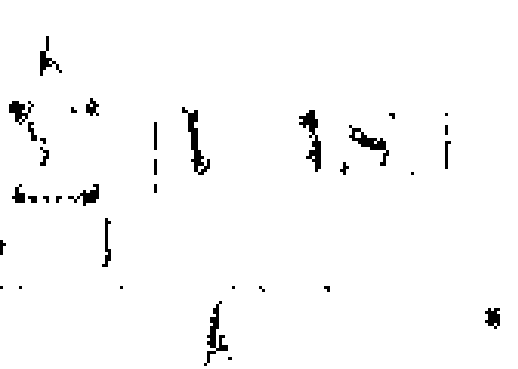
(2) UET scores, i.e.. absolute means of the (not absolute) evaluation scores on the nonneutral VASs, or 1,1, and (3) NT scores, i.e., the negative means of the (not absolute) evaluation scores on the nonneutral VASs, or ... High mET scores result from evaluations approaching the anchors of the scales and would imply very negative $\ldots m l, \ldots r$ very positive evaluations. High UET scores result from evaluations all approaching either the negative or the positive anchors of the scales; these scores would indicate that evaluations are all very negative $m$ very positive. High NT scores would reveal evaluations approaching the negative anchors of the scales signifying very negative evaluations. mET scores would be higher than UET scores, when evaluations would be mixed negative and positive, instead of all negative or all positive, suggesting (multidimensional) DT and not splitting.

\section{Procedure}

Participants were screened in one to six sessions with a biographical checklist, the SCID-interviews, and the BPDSI. If participants fulfilled the inclusion criteria, the RCT including this study was described to them and written informed consent was obtained.

Participants were tested individually. The current experiment was part of the second, sixth, and twelfth quarterly assessment of the RCT. The experiment started with participants receiving written instructions and broad information on the goal and procedure of the experiment. Next, participants filled out their baseline emotional state and were presented the first film clip. After having seen the clip, participants assessed their emotional state again and evaluated one to three characters of the film clip (direct evaluations) which were specified with labeled photos (e.g., 'Amalia', 'father'). The procedure including clip presentation, emotional state assessment, and character evaluation was repeated with the second, third, and fourth film clip, finally followed by an exit-interview. 


\section{CHAPTER 6}

The BPD Checklist was administered in the latter part of the measurement.

Presentation orders of the film clips were randomly chosen from a restricted set of (9936) potential orders. At the first assessment, each order of film clip type (e.g., clip with (1) victim, perpetrator, and helper roles, (2) positive roles, (3) ambiguous perpetrator roles, (4) neutral roles) was used at least three times, and each of the three film clip type versions was used at least 24 times within the sample. The order of film clip type at the second and third assessment was the same, but with randomly chosen other versions of the film clip types at the second and the remaining versions at the third assessment.

\section{Statistical analyzes}

Differences between evaluations of characters with negative, positive, and neutral roles, and changes in emotional states from baselines to post-film measurements were analyzed with one-tailed or, if indicated, two-tailed $t$-tests.

Some character evaluations were not collected according the described procedure (e.g., wrong film clip shown, refusal of watching certain film clip) and were removed. In secondary analyzes, we used corrected scores computed from evaluations of characters that were reliably categorized by the independent panel. Changes in evaluation and BPD Checklist scores were checked on outliers with the eye and on normal distribution with Komogorov-Smirnov tests. Pearson correlations were computed for changes in $\mathrm{mET}$, $\mathrm{UET}$, or NT scores for evaluations of the three role types (negative, positive, neutral) and BPD Checklist scores from $\mathrm{T} 1$ to $\mathrm{T} 2$, and from $\mathrm{T} 2$ to $\mathrm{T} 3$. Correlations that were significant were followed by computations of correlations for scores per distinct negative or positive roles (victim, perpetrator, ambiguous perpetrator, helper, intimate) and scale type.

mET scores were compared to the UET scores by one ANOVA per assessment with the factors Score Type (mET, uET), Role Type (negative, positive, neutral), and Scale Type (specific, nonspecific). A main effect of Score Type with higher $\mathrm{mET}$ scores than $\mathrm{UET}$ scores, would indicate that the evaluations of the characters were mixed negative and positive, and not totally negative or positive. 
INTERPERSONAL EVALUATIONS IN BPD DURING PSYCHOTHERAPY

\section{Results}

\section{Manipulation check}

\section{Negative evaluations of the role types}

Evaluations of the role types were in the hypothesized directions. NT scores for the negative and positive roles were respectively higher and lower than NT scores for the neutral role at all assessments, $|t| \mathbf{s}>9.51, p \mathbf{s}<.0005$. This indicates that participants evaluated the negative and positive roles respectively as more negative and positive than the neutral role. Analyzes of NT scores corrected for incorrectly categorized roles yielded similar results.

\section{Emotional effects of film clips}

In general, changes in reported emotional states from baseline to post-film assessment, were also in the intended directions. At all three assessments, participants reported lower positive emotion scores after the negative clips than at baseline, $t s<-4.91, p s<.0005$. At T3, participants further reported more arousal after the negative clips than at baseline, $t(42)=2.57, p<.01$. The positive clips were not followed by higher positive emotion scores than at baseline, $|t| \mathbf{s}<0.48, n s$. The positive clips however did result in lower arousal scores than at baseline at all three measurements, $t \mathrm{~s}<-2.0, p<.05$. Emotion scores after the neutral film clips were equal to baseline at $T 1, t(77)=1.32, n s$, two-tailed, but more positive than baseline levels at T2 and T3,ts $>2.1, p s<.05$, twotailed. Like the positive film clips, neutral film clips also induced lower arousal scores than at baseline at all three measurements, $t \mathrm{~s}<-3.25, p s<.005$, twotailed. A lack of increased arousal scores after the negative film clips, might be explained a posteriori by relatively high arousal scores at the baselines possibly resulting from apprehension and by reduced emotional expression (see also, Renneberg, Heyn, Gebhard, \& Bachmann, 2005). Lack of positive emotions after the positive clips, might also be due to the latter as well as their specific contents which may have induced negative affects.

\section{General treatment effects}

BPD Checklist scores generally decreased from T1 $(M=112, S D=29)$ to T2 $(M=97, S D=31), t(63)=4.25, p<.0005$; and from T2 $(M=101$, $S D=32)$ to $\mathrm{T} 3(M=85, S D=30), t(45)=4.27, p<.0005$. mET scores 


\section{CHAPTER 6}

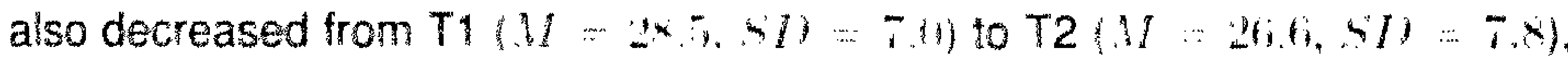

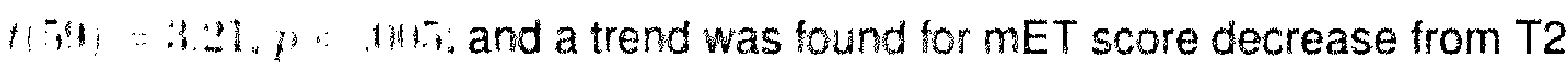

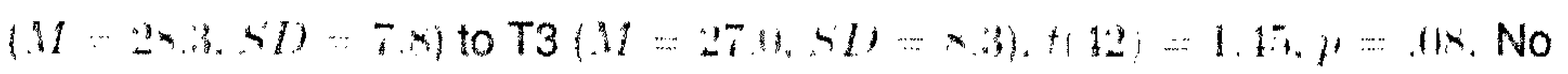
general decreases were found for the UET. and NT scores, neither from from

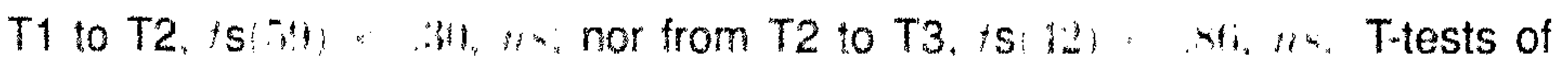
decreases of mET, UET, and NT scores calculated from evaluations of reliably categorized characters, showed a similar pattern.

\section{Correlations between decreases in BPD severity and decreases in extreme or negative evaluations}

Table 6.3 displays the correlations between decreases in original and corrected $m E T$, uET, and NT score from $T 1$ to $T 2$, and from $T 2$ to $T 3$ for negative, positive, and neutral role types, and concurrent decreases in BPD Checklist score.

Table 6.3: Pearson correlations between decreases in original and corrected ${ }^{1} \mathrm{mET}$, UET, and NT scores from T1 to T2 and from T2 to T3 for evaluations of the negative, positive, and neutral roles, and concurrent decreases in BPD Checklist score.

\begin{tabular}{|c|c|c|c|c|c|c|}
\hline \multirow[t]{3}{*}{ Score } & \multicolumn{6}{|c|}{$\triangle$ BPD checklist } \\
\hline & \multicolumn{2}{|c|}{$\mathrm{Tl} \rightarrow \mathrm{T2}$} & \multicolumn{4}{|c|}{$\mathrm{T} 2-\mathrm{I}_{3}$} \\
\hline & birmynat & correctant & $\mathrm{Ni}$ & 50 & con:merar & $N$ \\
\hline \multicolumn{7}{|l|}{$\Delta \mathrm{mET}$} \\
\hline negative & $.27^{*}$ & $.25^{*}$ & 64 & -.01 & .02 & 43 \\
\hline positive & $.39^{* * *}$ & $.42^{* * * *}$ & 64 & $.20^{\dagger}$ & $.20^{\ddagger}$ & 46 \\
\hline neutral & $.17^{\dagger}$ & $.22^{*}$ & 61 & .19 & $.31^{*}$ & 45 \\
\hline \multicolumn{7}{|l|}{$\triangle \mathrm{UET}$} \\
\hline negative & .08 & .05 & 64 & -.11 & -.05 & 43 \\
\hline positive & $.18^{\dagger}$ & $.20^{\dagger}$ & 64 & .10 & .12 & 46 \\
\hline neutral & .02 & .03 & 61 & -.02 & -.01 & 45 \\
\hline \multicolumn{7}{|l|}{$\triangle N T$} \\
\hline negative & -.30 & -.25 & 64 & $.24^{\dagger}$ & $.24^{\dagger}$ & 43 \\
\hline positive & -.16 & -.16 & 64 & -.10 & -.13 & 46 \\
\hline neutral & .02 & -.02 & 61 & .03 & -.03 & 45 \\
\hline
\end{tabular}




\section{Multidimensional extreme evaluations}

Decreases in $\mathrm{MET}$ score for the positive characters correlated relatively strongly with concurrent decreases in BPD Checklist score from $\mathrm{T} 1$ to $\mathrm{T} 2$. Correlations for $\mathrm{mET}$ score decreases for the negative roles and neutral roles from $\mathrm{T} 1$ to $\mathrm{T} 2$ were somewhat less strong. Not all correlations between mET score decreases from $\mathrm{T} 1$ to $\mathrm{T} 2$ for the separate negative and positive roles and concurrent BPD Checklist score decreases appeared significant. Significant correlations were

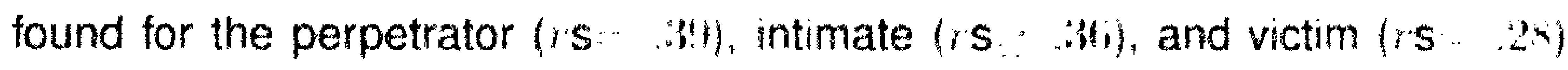

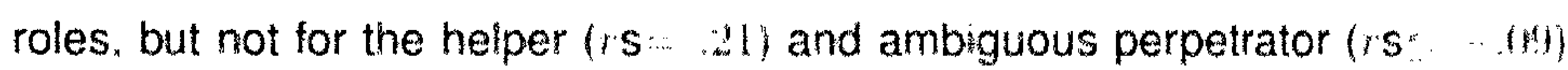
roles. Correlations between decreases in original and corrected mET scores and concurrent decreases in BPD Checklist scores further not only resulted from $\mathrm{mET}$ score decreases on specific scales. The correlations of score decreases for negative, positive and neutral characters were significant for respectively all scale types $(r s, 23), 1 s, 115$, the nonspecific and neutral scale types $(r s, 2 \mathrm{x}), r \mathrm{~s},(0)$, and for the specific scale types $(r s, 23), p s$

From T2 to T3, correlations for decreases in MET scores for the positive and negative characters were no longer significant. Only the $\mathrm{mET}$ score decreases for neutral characters, at least the corrected mET score decreases, remained significantly, though modestly, correlated with BPD Checklist score decreases.

Figure 6.1 shows the (corrected) $\mathrm{mET}$ scores at $\mathrm{T} 1$ and $\mathrm{T} 2$ and/or $\mathrm{T} 2$ and T3 for the perpetrator, intimate, victim, and neutral role of subgroups of patients with large decreases, medium decreases, or small/no decreases in BPD symptom levels. These subgroups were split on the basis of decreases in BPD Checklist score from $T 1$ to $T 2$ and from $T 2$ to $T 3$, corresponding with cumulative percentages of 33 and 67 . Note that the subgroup with the highest $\mathrm{mET}$ scores at $T 1$ showed the largest decreases in $\mathrm{MET}$ scores from $\mathrm{T} 1$ to $T 2$ of the three groups, $F \mathrm{~S}(4.55) \geq 3.10,1 \mathrm{~s} \leq .(12$, and $t \mathrm{~s} \geq 2.16,1 \mathrm{~s} \leq .12$.

\section{Unidimensional extreme evaluations}

Decreases in original and corrected UET score for the negative, positive, or neutral role types during the first and second half of treatment, did not correlate significantly with concurrent decreases in BPD Checklist score. 


\section{Multidimensional Extreme Thinking}
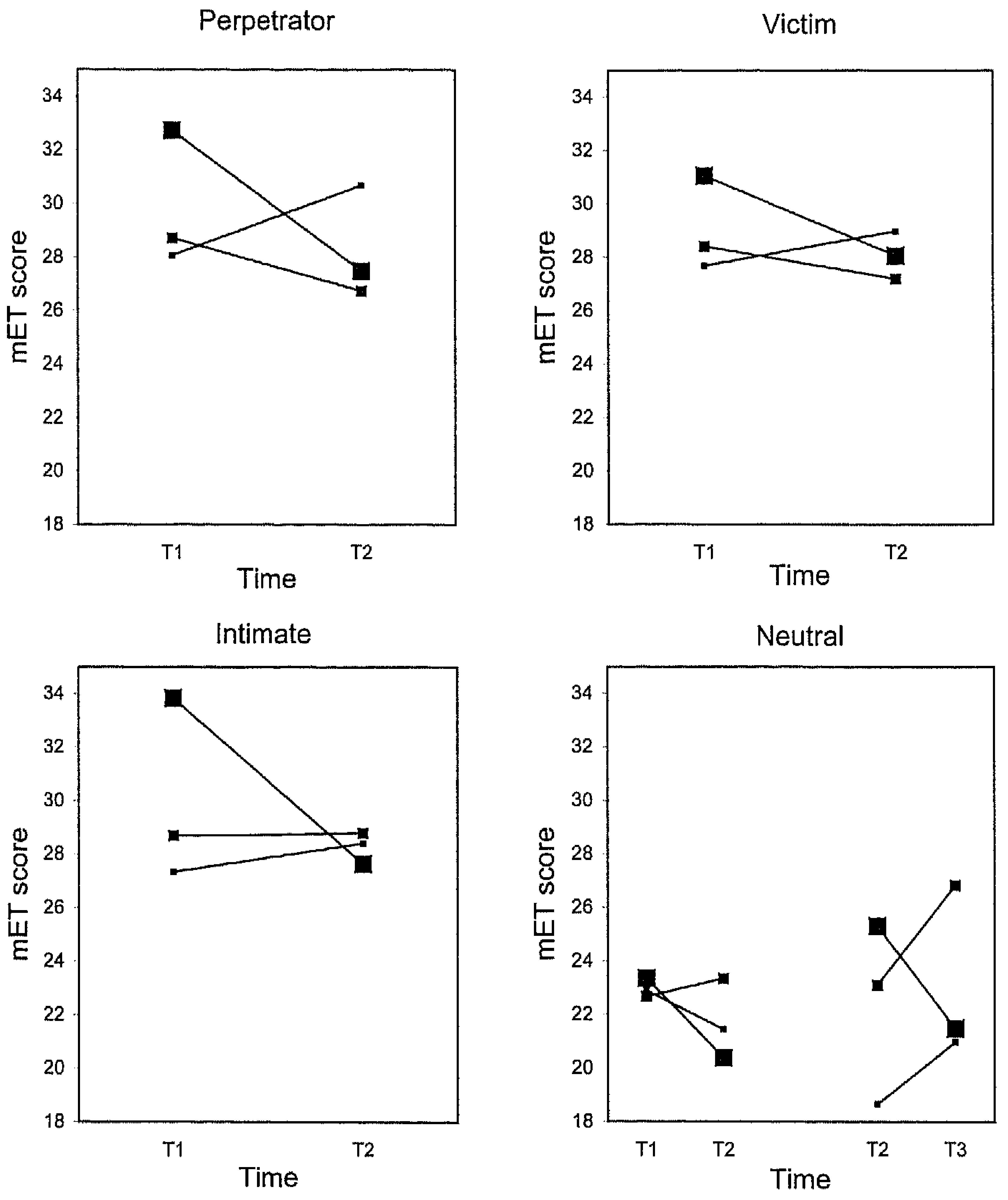

$\rightarrow$ small/no $-\triangle \mathrm{BPD}$
$\rightarrow-$ medium $-\triangle \mathrm{BPD}$
$\rightarrow$ - large $-\triangle \mathrm{BPD}$

Figure 6.1: Corrected mET scores of BPD patients with small/no, medium, and large decreases in BPD Checklist score for the perpetrator, victim, intimate, and neutral roles during the first and/or second half of treatment. 


\section{INTERPERSONAL EVALUATIONS IN BPD DURING PSYCHOTHERAPY}

\section{Negative evaluations}

Decreases in original and corrected NT score and BPD Checklist score did not show significantly positive correlations for any of the role types and treatment halves. Analyzes for the first half of treatment however did yield significantly negative correlations between NT score decreases for the negative roles and BPD Checklist score decreases. Further explorations of correlations for the distinct roles and scale types revealed a borderline significant negative correlation $(r s=-.24), p s=.05$ (two-tailed test), for the victim role. Correlations for the other roles and the distinct scale types were not significant.

Analyzes for the second half of treatment showed that this correlation between NT score decreases for the negative roles and BPD Checklist score decreases had changed into a borderline positive correlation. Further explorations of correlations for the distinct roles and scale types revealed a significant correlation $(r s=.30), p s<.05$, for the victim role which was significant for the specific scales $(r s=.37), p<.01$, but not for the nonspecific scales $(r s=09$.$) , ns.$

\section{Controls for depression}

Dichotomous or negative interpersonal evaluations are thought to characterize patients with BPD as well as depressive patients (e.g., Beck, 1979). We therefore performed five linear regression analyzes with decrease in baseline sadness score and concurrent decrease in BPD Checklist score from T1 to T2, or from T2 to T3 as independent variables, and as the dependent variables the concurrent mET or NT score decreases that had appeared to correlate significantly or borderline significantly with decreases in BPD Checklist score. We performed the analyzes for corrected and uncorrected scores. Most regression coefficients for decreases in BPD Checklist score appeared to be significant $(.16<|\beta| \mathbf{s}<.42), .005<p s<.12$, in contrast with none of the $\beta$ s for decreases in sadness scores $(.004<|\beta| \mathbf{s}<.19), .06<p \mathbf{s}<.49$. These results indicate that the correlations did not result from decrease of sadness, reducing the probability that decrease of ET and NT resulted from decrease of depression.

\section{Controls for attrition}

In order to control for bias resulting from the patients who would drop out between T1 and T2 $(n=14)$ or T2 and T3 $(n=18)$, correlations for score decreases 


\section{CHAPTER 6}

during the first half of treatment were also calculated without these drop outs.

Correlations between decreases in mET scores for the positive roles and neutral roles and decrease in BPD Checklist score from $T 1$ to $T 2$ also remained significant without drop outs. Correlations between and mET or NT score decrease for the negative roles and BPD Checklist score decrease from $T 1$ to $T 2$, were not significant in this group, though comparably strong.

\section{Discussion}

This study demonstrated a decrease of ET during psychotherapy and a positive relation with concurrent decline in BPD symptom level, independent of indications for decrease of depression. These findings are in line with the model that treatment reduces $E T$ in patients with $B P D$ and that a reduction of ET leads to decrease of BPD severity (Arntz, 2004; Pretzer, 1990), though of course a real causal test needs inclusion of a non-treatment group and a controlled focused manipulation of ET. This longitudinal study suggests that ET is characteristic of BPD and/or BPD-related features that change during therapy, and not of other, i.e., non-pathological features of persons suffering from BPD (e.g., an emotional temperament) that were not controlled in previous, cross-sectional studies (e.g., Veen \& Arntz, 2000). A further notable finding was that patients showing the largest scores for ET at treatment start showed largest decreases in ET and BPD symptom level during the first treatment half. Maybe, patients who are more open to emotions and to tendencies to think extremely compared to more detached patients, are more accessible for treatment as well.

The borderline patients did not show a decrease of NT during therapy, nor did a decrease of NT significantly and positively correlate with concurrent decrease in BPD symptom level. ET may be more central to BPD than NT. Alternatively, the present treatments may have been more successful in changing dichotomous thinking than in reducing negativity, which may pose a future challenge for improvement of treatments. An unexpected and puzzling finding was a negative correlation between decrease in NT about negative characters, particularly abused child characters ('victim' role), and concurrent BPD symptom level decrease during the first treatment half. A speculative interpretation is that the increase of negative evaluations of these characters by the borderline patients reflects an increased recognition of their own deprived childhoods. This change might be a prerequisite for subsequent improvement.

The evaluations of the borderline patients that became less extreme during 
therapy, could not be conceived of as manifestations of splitting, for the extreme evaluations of the patients across all conditions at start, during and end of therapy were all mixed negative and positive part evaluations and only decreases of these multidimensional evaluations were significantly positively related to decrease of BPD severity. Patients with BPD seem neither incapable of giving relatively differentiated evaluations (see also, chapters 4 and 5 of this thesis; Benjamin \& Wonderlich, 1994; Veen \& Arntz, 2000; Westen et al., 1990a, 1990c), nor stuck at an immature unidimensional developmental level (see also, Westen et al., 1990b, 1990c, 1990d). This does not mean that patients with BPD always use their full capabilities (see, Arntz \& Veen, 2001), or that their evaluations are coherent and accurate (see, Stuart et al., 1990).

Schema relatedness of characters and/or evaluations did not clearly differentiate the pattern of correlations between decrease in ET and BPD. Schema related extreme evaluations of schema related characters therefore appear not to be generally indicative of BPD pathology. During the first half of treatment, however, the correlations for schema related characters were somewhat higher than those for schema unrelated characters. This may imply that extreme interpersonal evaluations activated by schemas are addressed first in therapy, though these higher correlations may also be due to initial values: ET scores for evaluations of the schema related characters at start of therapy were much higher than schema unrelated characters.

Regarding the time line, larger correlations between decrease of ET and concurrent BPD level during the first than second half of the treatments indicate that BPD relevant change of ET is particularly realized during the first half of treatment, similarly as decline in BPD symptom level and general psycho- and personality pathology of these patients (see, Giesen-Bloo et al., 2006b). During the remaining treatment time, only decrease of extreme evaluations of film characters with neutral roles showed a significant and only moderate correlation with decrease in BPD pathology level. Differences between the first and second half of treatment did not result from attrition of patients, on the contrary, the pattern was even stronger without drop outs. In order to improve our understanding of the mechanism of change in BPD and how to optimize this change, future studies should include more frequent measurements.

This study had several strengths. First, the participants were reliably and validly diagnosed with semi-structured interviews. Second, the sample was representative of the outpatient BPD population, showing moderate to severe levels of BPD. Third, the dependent variables ET and NT were not assessed 


\section{CHAPTER 6}

by self-report measures, but with an experimental task consisting of valid and reliable manipulations of the independent stimuli (characters and scales) as tested by judgments of an independent panel and checked by the participants evaluations of the characters and emotional reactions on the films. Although the task has been administered only once per participant in previous studies, we think that the different task versions, the low frequency of administration, persistent correlations from $\mathrm{T} 1$ to $\mathrm{T} 2$ and the interpretable outcomes provide a sufficient safeguard against increased strategic responses and lower scores for ET due to task repetition and therapeutic techniques resembling the task (e.g., multidimensional scaling in SFT). A fourth strength of this study is the large power through inclusion of a large number of patients undergoing longterm treatment aiming at basic change. Finally, the longitudinal study design with within-subject comparisons provide better control of third variables and demonstrate more feasible changes in patients with BPD than previous crosssectional studies.

The following limitations have to be taken in account. First, it is not certain whether the scores for ET and NT accurately represent the extreme and negative evaluations of borderline patients. The characters were emotionally appealing, but it is not certain whether they were sufficiently appealing and specific to induce the interpersonal evaluative style which is shown outside the laboratory and whether the VASs and scores captured these optimally. Second, the character stimuli varied on the targeted factors, but, being not created for this experiment, they had other irrelevant characteristics as well (such as gender, celebrity, culture) which increased noise and decreased power. Third, ET was not assessed frequently enough to test the time relation with reduction in BPD manifestations in detail. Fourth, the strength of the significant associations were all modest (i.e., . $21 \leq|r| \mathbf{s} \leq .42$ ). Extreme and negative interpersonal evaluations may not directly lead to extreme and negative behaviors towards others, but might be tempered in several ways, for example by dissociation (see also Stiglmayr, Shapiro, Stieglitz, Limberger, \& Bohus, 2001), thought suppression (Rosenthal, Cheavans, Lejuez, \& Lynch, 2005), and impulse control. Further research is needed to investigate the interplays between these factors in BPD and how these change in treatment. The moderate associations are further supportive of the idea that ET is important, but not the only factor underlying BPD. Finally, the study did not include a no-treatment control condition, which is necessary for a definite attribution of change to treatment (though the correlation between ET and BPD changes are indicative). Although inclusion of a 
three-year no-treatment control group would have been unethical, a time-limited waiting-list group or a group of patients undergoing supportive treatment, might have been alternatives.

In sum, decrease of interpersonal extreme evaluations appeared to be related to decrease of BPD pathology and might thus be an important element of change in BPD. Future research should focus in more detail on mechanisms of change and how to optimize these. Clinically, these findings suggest that addressing processes causing ET might be important in treating BPD. Furthermore, the finding that high initial levels of ET are related to treatment success might be helpful in treatment indication. Alternatively, it might indicate that treatment methods should be adapted for those BPD patients that don't show high levels of ET. 
Hypervigilance and Extreme Evaluations in Borderline Personality Disorder:

\section{General Discussion}




\section{CHAPTER 7}

\section{Introduction}

This thesis focuses on hypervigilance and extreme evaluations in BPD - two central aspects in cognitive models of this disorder. The preceding chapters have introduced the research questions and described five experimental studies investigating hypervigilance and extreme evaluations with cross-sectional and longitudinal designs. This final chapter contains a summary and discussion of the findings. It elaborates on the theoretical and clinical implications of the findings and presents recommendations for future research and clinical praxis. It further reflects on the limitations of the studies as well as on some general methodological aspects of experimental research on BPD.

\section{Summary of the findings}

The findings of the studies are summarized by the following answers to the research questions.

\section{Hypervigilance}

\section{Is BPD characterized by hypervigilance?}

BPD appeared to be persistently characterized by hypervigilance for emotional stimuli (see chapters 2 and 3). Patients with BPD showed more hypervigilance (for certain emotional stimuli) than patients with an Axis I or Cluster $C$ personality disorder and the relation between BPD and hypervigilance remained significant when controlled for comorbid anxiety, depressive and cluster $\mathrm{C}$ personality disorder (see chapter 2).

\section{What kind of hypervigilance is characteristic of BPD?}

Across the studies, patients with BPD showed hypervigilance for various stimuli. One study found more hypervigilance in BPD as compared to Cluster $C$ personality disorder, Axis I disorder and no disorder for emotionally negative stimuli related to BPD-schemas (i.e., "I am powerless", "I am inherently unacceptable", "The world is / others are malevolent") and for emotionally positive stimuli in general. This study also found a trend for more hypervigilance in patients with BPD (or an Axis I disorder) as compared to patients with a Cluster 
C personality disorder and persons without disorders for schema-related emotionally negative subliminal stimuli (see chapter 2). Another study, investigating only effects of emotionally negative stimuli, found more hypervigilance in patients with BPD as compared to healthy controls for both schema-related and schema-unrelated stimuli (see chapter 3 ).

\section{What are potential predictors of hypervigilance in BPD?}

Hypervigilance for schema-related emotionally negative and positive stimuli appeared to have different correlates. Hypervigilance for schema-related negative stimuli was predicted by higher levels of BPD-related anxiety symptoms and sexual childhood trauma. Hypervigilance for schema-related positive stimuli was predicted by higher levels of BPD-related identity disturbance symptoms (see chapter 2).

\section{Is successful treatment of BPD related to decrease of hypervigilance?}

Successful treatment of BPD appeared to be related to decrease of hypervigilance. After three years of psychotherapy aiming at recovery from BPD, hypervigilance was completely reduced to normalized levels in recovered patients, but not in non-recovered patients (see chapter 3 ).

\section{Extreme evaluations}

\section{Is BPD characterized by extreme evaluations?}

Patients with BPD were characterized by extreme (negativistic) evaluations. These evaluations were found to be specific for patients with BPD as compared to patients with other personality disorders, such as a cluster $C$ personality disorder or antisocial personality disorder (see chapters 4 and 5).

\section{What kind of extreme evaluations are characteristic of BPD?}

BPD appeared to be characterized by negative evaluations. Persons with BPD evaluated schema-related malevolent persons and schema-unrelated neutral persons more negatively than healthy controls, and schema-related benevolent persons more negatively than patients with Cluster $C$ personality disorder and healthy controls. These negative evaluations appeared to be independent of indications of depression (see chapter 4). Patients with BPD even 


\section{CHAPTER 7}

showed more negative evaluations of emotionally negative non-interpersonal stimuli compared to persons with antisocial personality disorder and healthy controls (see chapter 5). Patients with BPD did not clearly show more 'multidimensional' (mixed negative and positive) or 'unidimensional' (all negative or positive) dichotomous evaluations compared to personality disorder controls and/or healthy controls (see chapters 4 and 6 ).

\section{What are potential predictors of extreme evaluations in BPD?}

The correlations between childhood trauma types and negativistic evaluations differed for schema-related emotionally negative and schema-related emotionally positive interpersonal stimuli. Negativistic evaluations of schema-related emotionally negative persons were (partially) predicted by severity of childhood sexual abuse, whereas negativistic evaluations of schema-related emotionally positive persons were not predicted by childhood sexual abuse or other childhood traumas (see chapter 4)

\section{Is successful treatment of BPD related to decrease of extreme evalua- tions?}

Decrease of BPD-symptoms during treatment appeared to be related to decrease of extreme interpersonal evaluations. Moderate to strong positive correlations were found for decrease of multidimensional dichotomous evaluations during both first and second half of treatment, but particularly the first half of treatment. Negativistic evaluations did not clearly decrease concurrently with BPD pathology, although correlations between reductions of BPD-symptoms and negativistic evaluations of negative stimuli during the second treatment half did tend to significance. Instead, an unexpected correlation between decrease of BPD-symptoms and increase of negativistic evaluations of schemarelated emotionally negative stimuli (particularly persons in abused child roles) appeared during the first treatment half (see chapter 6 ).

\section{Discussion of the findings}

\section{Hypervigilance}

The studies in this thesis persistently showed that hypervigilance is characteristic of BPD. This finding is in accordance with other studies on hypervigilance 
in BPD (Arntz et al., 2000; Waller \& Button, 1999). Considering the tight relation between hypervigilance and clinical anxiety (Harvey, Watkins, Mansell, \& Shafran, 2004; Kindt \& Van den Hout, 2001), the result is also in line with already the earliest descriptions of borderline pathology. Hoch and Cattell (1959) for example wrote:

"[In borderline patients] there is a low threshold to the anxiety response, with a low threshold to experiencing it, and bewilderment in coping with it."

Other findings consistent with this anxious side are remarkably high comorbidities of BPD with anxiety disorders and anxious cluster personality disorders (Zanarini et al., 1998b, 1998c; Zimmerman \& Mattia, 1999), high rates of childhood trauma in BPD (e.g., Sabo, 1997), and anxiety-related experimental findings on BPD such as increased amygdala activation (Herpertz et al., 2001a) and normalized amygdala activation after successful treatment (Arntz, Gielen, van der Heijden, Cima, \& van der Veen, 2008b; Schnell \& Herpertz, 2007). Framing BPD as anxiety disorder is still uncommon, perhaps because patients with BPD do not always clearly show hyperarousal on peripheral indices (Herpertz et al., 2000, 2001b; Renneberg et al., 2005) although some studies did find evidence for peripheral hyperarousal (Arntz et al., 2008b; Lobbestael \& Arntz, 2008). Viewing BPD as anxiety disorder therefore provides new perspectives for research and clinical praxis.

The precise conceptualization of hypervigilance in BPD remained somewhat unclear as stimulus type manipulations and correlational analyses did not yield unequivocal support for one of the three investigated theoretical models. The Beckian hypothesis of BPD schema-related hypervigilance was supported in a first study (see chapter 2), but could not be replicated in a second study (see chapter 3), which showed hypervigilance for both schema-related and schemaunrelated stimuli. Vice versa, the idea of a general hypervigilance in BPD following from a general emotional dysregulation hypothesized by Linehan (1993) was supported in the second, but not the first study which did not show generalized hypervigilance, but a schema-related hypervigilance and different correlates for hypervigilance for negative and positive stimuli.

The findings of this thesis provide best evidence for the trauma model, explaining hypervigilance in BPD as an anxiety-related post-trauma phenomenon. Hypervigilance, at least hypervigilance in the context of emotionally negative stimuli, was correlated with BPD related anxiety symptoms and childhood sexual abuse. A tendency to show vigilance for subliminally presented negative 


\section{CHAPTER 7}

stimuli was further shared with patients having anxiety disorders. The latter finding is in accordance with the idea that better functioning anxious individuals have the capacity to suppress hypervigilance at a more strategic level, but not at a more automatic level (see Williams et al., 1997).

Trauma and/or anxiety appeared not a logical explanation of the hypervigilance in BPD for emotionally positive stimuli. Hypervigilance for emotionally positive stimuli tended to correlate negatively with BPD anxiety symptoms and did not correlate with childhood sexual or physical abuse but did correlate positively with BPD identity disturbance symptoms. One of the following speculative hypotheses might account for these findings. (1) Patients with pronounced BPD identity disturbance symptoms have a prominent negative self-schema compared to the malevolent other-schema, resulting in a net urge to approach stimuli related to a strong/good/caring self or other. Similar effects have been observed in persons who had fasted for 18-24 hours and showed appetitive attentional biases, i.e. biases towards positively evaluated food stimuli (Lavy \& van den Hout, 1993; Mogg, Bradley, Hyare, \& Lee, 1998). (2) Identity disturbed patients with BPD attend to positive emotional words as an attempt to avoid the processing of (negative) emotional stimuli (see also Lavy \& van den Hout, 1994). This bias is only evident for supraliminally presented stimuli as this is a mood regulation strategy and not an automatism (see also Mogg et al., 1993). (3) Patients with pronounced BPD identity disturbance symptoms have a strong tendency to avoid positive self- or other-descriptions because these are incompatible with the negative self- and other-schemas of these patients. Psychodynamic theorists assume, the combination of positive and negative representations trigger an intolerable fear of the annihilation of negative representations by positive representations (Kernberg, 1996). A study assessing biases both towards and from positive and negative, subliminal and supraliminal stimuli, and differentiating self and other related stimuli can test these hypotheses.

Although it is too early for firm clinical implications, the hypervigilance findings in BPD do suggest the following. First, finding hypervigilance in BPD independent of comorbid anxiety disorders underlines the importance of anxiety and/or trauma interventions in the treatment of patients with BPD reporting anxiety symptoms and/or sexual or physical childhood abuse, regardless of the presence of a post-traumatic stress or other anxiety disorder. Normalized hypervigilance after schema- or transference-focused psychotherapy (this thesis) and reduced amygdala hyperactivation after dialectical behavior therapy (Schnell \& Herpertz, 2007) suggest that various treatment strategies may be 
potentially effective. Further investigation could focus on finding the most efficient interventions. Second, not finding convincing support for schema-related hypervigilance in BPD casts some doubt upon the effectiveness of correction of the investigated schemas in order to overcome hypervigilance. Future research should specify the schemas causing anxiety in BPD. Third, support for a differentiated kind of emotional dysregulation, with different correlates of hypervigilance for negative and positive stimuli, instead of a general emotional dysregulation in BPD (this thesis) and evidence of emotional competences (such as accurate emotional sensitivity and goal-oriented behavior) instead of emotional deficits (Gratz, Rosenthal, Tull, Lejuez, \& Gunderson, 2006; Lynch et al., 2006) point to rather specific instead of general emotional problems in BPD. A treatment focused on these specific problems and using these strengths seems therefore more adequate than a training focused on general emotional regulation skills.

\section{Dichotomous evaluations}

The studies of this thesis did not provide clear support for the hypothesis that making more extreme or 'dichotomous evaluations' characterizes BPD. Decrease of BPD pathology during treatment appeared to be positively related to decrease of dichotomous interpersonal evaluations scores (see chapter 6), showing that dichotomous thinking is sensitive to treatment and characterizes BPD pathology, and not other features of borderline patients, such as emotional temperament or emotional sensitivity. However, the cross-sectional study of this thesis did not show pronounced dichotomous evaluations in patients with BPD as compared to patient and non-patient controls (see chapter 4). The first finding is consistent with other cross-sectional studies which demonstrated more dichotomous evaluations of film characters and counselors in patients with BPD compared to patients with cluster $C$ personality disorders and non-patient controls (Veen \& Arntz, 2000; Ten Haaf \& Arntz, 2009). The second finding is not consistent with these previous studies.

The following explanations of the varying outcomes with regard to dichotomous evaluations in BPD seem plausible. First, relatively high dichotomous thinking levels of the control participants in the cross-sectional study of this thesis compared to those of the other studies (i.e., Ten Haaf \& Arntz, 2009; Veen \& Arntz, 2000) may have been confounds of relatively low intelligence levels. The control groups were matched on educational level to the BPD group but 


\section{CHAPTER 7}

may not have been as intelligent because educational level probably underestimates intelligence in BPD. Intelligence has also been shown to correlate with other cognitive characteristics associated with pathology (e.g., poor metacognitive performance; Arntz, Bernstein, Oorschot, Robson, \& Schobre, 2008a) and might very well also correlate with dichotomous thinking. Future cross-sectional studies on dichotomous thinking should therefore match participant groups on intelligence. Second, the relatively high dichotomous thinking levels of the nonpatient control groups of the cross-sectional study of this thesis compared to those of the other studies may in part also be a valid finding, resulting from a careful matching non-patient controls (e.g., not only students). It is not the first time that a non-patient control group shows dichotomous evaluations (see reports of 'split' inkblot interpretations in non-patients of Leichsenring, 1999). This explanation indicates that dichotomous thinking may not necessarily be pathological! Third, relatively low and high levels of personality psychopathology (i.e., BPD-symptom level and/or number of personality disorders) of the BPD patients and patient controls respectively in our cross-sectional study compared to those in the two studies which found BPD specific dichotomous evaluations may finally have caused lower and higher dichotomous thinking levels of the BPD patients and patient controls in our study (Ten Haaf \& Arntz, 2009; Veen \& Arntz, 2000). Making dichotomous evaluations might be specific of patients with only high levels of BPD pathology or, perhaps, with high levels of general personality pathology.

In two of the three studies, borderline pathology appeared to be particularly marked by more extreme evaluations which were mixed negative and positive, schema-related and-unrelated, and interpersonal. Other experimental studies also demonstrated mixed negative and positive evaluations of interpersonal stimuli in BPD (Bonis et al., 1998; Leichsenring, 1999; Ten Haaf \& Arntz, 2009; Veen \& Arntz, 2000). In contrast, spontaneous evaluations of borderline patients were found to be unmixed and mainly negativistic (Arntz \& Veen, 2001; Westen et al., 1990a). These evaluations may however have only partially represented all (mixed) evaluations of the borderline patients, for example due to lack of motivation to be more comprehensive. In contrast with Veen and Arntz (2000), the studies of this thesis (see chapters 4, 5, and 6) did not find schemaspecific extreme evaluations in BPD. Maybe, the results of the first study has been confounded by differences in emotionality and liveliness of the schemarelated and -unrelated film clips. Extreme evaluations of non-interpersonal stimuli of patients with BPD, for which no significant effects were found, have not 
been examined elsewhere.

The kind of extreme evaluations found in BPD is not in accordance with the psychodynamic concept of splitting of Kernberg (1996). This defense mechanism would result in unmixed, i.e., 'all negative' (devaluating) or 'all positive' (idealizing), evaluations. Dichotomous evaluations of schema-unrelated stimuli and on schema-unrelated scales are neither in line with cognitive models hypothesizing schema-related evaluations (Pretzer, 1990) or evaluations resulting from schema-activation (Layden et al., 1993), at least not for the schema's investigated in the experiments of this thesis. As mixes of concurrent negative and positive aspects represent a high developmental level (see, Harter, 1986), evaluations of borderline patients also did not appear to reflect regressions to developmentally primitive levels of functioning (Layden et al., 1993). Among the hypothesized conceptualizations, the Beckian concept of the cognitive distortion of dichotomous or polarized thinking (Beck, 1976; Pretzer, 1990) seems most consistent with the demonstrated evaluations. Concurrent extreme negative and positive evaluations are then regarded as polarized evaluations on several dimensions (e.g., very handsome and very malevolent) (see also, Veen \& Arntz, 2000). The multidimensionality of the dichotomous evaluations of borderline patients may reflect a tendency to judge others in integrative and balanced ways and/or to temper initial negative interpretations ('complex affectdriven thinking'; see, Bradley \& Westen, 2005, and next section on negativistic evaluations).

Various hypotheses exist on the role of dichotomous thinking in borderline pathology. Beck (1976) and Pretzer (1990) hypothesize that dichotomous thinking is primarily maladaptive and plays a causal or maintaining role in BPD. Dichotomous thinking would make individuals more vulnerable to, for example, depression, mood swings and interpersonal problems. According to Kroll (1988, p. 33), dichotomous thinking or related 'disturbances' such as 'exaggeration', 'loss of relevant detail' and 'impressionistic and global perception' may also be maladaptive consequences:

"Stress beyond a tolerable level interferes with the logical thinking of all persons; it is only a matter of degree that separates borderlines from others. Hold a gun to my head and ask me to list rapidly the last ten novels (with authors) which I have read and I suspect we will see both amnesia and depersonalization."

Other theorists have elaborated the adaptive aspects of dichotomous evaluations. For example, Plummer and Das (1973, p. 294) wrote: 


\section{CHAPTER 7}

"Dichotomous thinking allows the individual to organize his value system in a simplified 'all-or-none' manner. A particular extreme alternative will consequently stand out clearly as more appropriate than the others, thus making a quick decision [in favor of some action] possible."

Following this line of thinking, borderline patients might dichotomize their evaluations in order to facilitate responding to subjectively experienced threat, such as threat related to childhood trauma and/or to the experience of very dysphoric states (Zanarini et al., 1998a). The more recently formulated cognitive 'mode' model of Beck (1996) similarly views dichotomous thinking as part of an inborn 'primal mode' which was adaptive in ancient dangerous environments but is mostly counterproductive nowadays. Patients with BPD might finally apply dichotomous thinking to facilitate action in order to counterbalance unpleasant feelings of under-arousal or emptiness (see BPD criterium 7 of the DSM-IV: American Psychiatric Association, 2000). This explanation has also been put forward for the increased use of the cognitive distortions of personalization and overgeneralization in individuals scoring high on the personality characteristic of affect intensity (Dritschel \& Teasdale, 1991; Larsen, Diener, \& Cropanzano, 1987). Patients with BPD might thus not only suffer from dichotomous thinking, but also benefit from it. Future research should aim at increasing the understanding of the use of dichotomous thinking in BPD, for example by investigating (temporal) relations between (state) anxiety or under-arousal and dichotomous thinking.

Clinically, the findings with regard to dichotomous evaluations in BPD do not warrant the labeling of extreme evaluations in borderline patients as the primitive defense of splitting. Therapists who persistently see splitting might overgeneralize the extreme evaluations of BPD patients to 'all bad' and 'all good' evaluations under the influence of their own strong emotions (fear, anger) in therapies with borderline patients (see further, Veen \& Arntz, 2000). The results further imply that treatment of these extreme evaluations is possible and probably useful. Although a correlation between decreases of BPD pathology and dichotomous evaluations does not mean that dichotomous evaluations cause BPD pathology, it does seem logical to assume that these kind of evaluations at the least hamper the relationships of BPD patients. The most adequate treatment approach on the basis of the available data seems to consist of uncovering the tendency to making dichotomous evaluations including its dysfunctionality, investigating the precursors of these evaluations (such as experiencing stress 
or underarousal), and replacing these evaluations by more functional ones. Future research on the relation between dichotomous thinking and feelings of anxiety or emptiness may add other beneficial techniques focused at reduction of these dysfunctional extreme evaluations.

\section{Negativistic evaluations}

Negativistic evaluations appeared to be a pervasive characteristic of BPD in this thesis (see chapters 4,5 , and 6 ). This finding is in line with clinical observations of strong tendencies towards 'victimization' and 'suspiciousness' in this patient group (e.g., Kroll, 1988), as well as with many empirical studies on BPD finding negativistic interpersonal interpretations and evaluations of a broad array of stimuli (e.g., film characters, parents, hospital staff, other patients, ambiguous social situations, facial expressions, ink blots) (Ackerman et al., 1999; Arntz \& Veen, 2001; Baker et al., 1992; Barnow et al., 2009; Benjamin \& Wonderlich, 1994; Kurtz \& Morey, 1998; Lerner \& St. Peter, 1984; Nigg et al., 1992; Segal et al., 1992; Stern et al., 1997; Stuart et al., 1990; Wagner \& Linehan, 1999; Westen et al., 1990a, 1990b, 1990c, 1990d), lower 'likability' of ambivalent and neutral non-interpersonal stimuli (Kurtz \& Morey, 1998), reduced facial reactivity to positive stimuli (Renneberg et al., 2008), and higher levels of negative affect (Levine et al., 1997; Rosenthal et al., 2005; Stein, 1996; Zanarini et al., 1998a). The relative persistence of negativistic thinking in BPD during longterm treatment (see chapter 6 ) is consistent with findings of continued reduced smiles in borderline patients responding to good-feel movies during and after emotional crisis (Renneberg et al., 2008) and of similar levels of malevolent interpersonal evaluations in adolescent and adult patients with BPD (Westen et al., 1990d).

The following speculative hypotheses on the origins of negative thinking in BPD are theoretically appealing and consistent with the data to date. First, negativistic thinking may result from the combination of negative affectivity / emotional dysregulation and disinhibition, two personality traits which have been shown to characterize (features of) BPD and/or which may predispose individuals to BPD (e.g., Trull, 2001). Difficulty in self-soothing abilities, rooted in genetic vulnerabilities and/or neglectful or abusive early caretaking (e.g., Baker et al., 1992; Nigg et al., 1992; Westen, 1990), may be part of this combination of personality traits. This hypothesis is in accordance with the generalness of negativistic thinking, for example in both interpersonal and non-interpersonal 


\section{CHAPTER 7}

contexts (see chapters 4 and 5), and with its persistence, for example after therapeutical interventions (chapter 6 of this thesis, Renneberg et al., 2008). Second, negativistic evaluations in BPD may also reflect negative schemas resulting from negative interpersonal experiences. These experiences may partly be traumatic. For example, borderline patients showed most negative evaluations of negative schema-related interpersonal stimuli, particularly persons with negative abusive roles, and these evaluations were related to childhood sexual abuse (chapter 4 of this thesis, Nigg et al., 1991). Most specific for BPD however, were less positive evaluations of persons with positive caring partner roles, which appeared to be unrelated to childhood trauma (chapter 4 of this thesis, Nigg et al., 1992). Possibly, other interpersonal learning experiences than clear-cut childhood trauma, such as more subtle negative interactions with primary caretakers, result in negativistic thinking of patients with BPD. The specific less positive evaluations may also represent a general personality trait (e.g., negative affectivity) which simply yielded the strongest group contrasts for positive stimuli. A final origin, at least of the less positive evaluations of persons with caring roles in BPD, might be a dissociative, detached attitude in these patients. Dissociative symptoms characterize many patients with BPD (American Psychiatric Association, 2000). Minor dissociation or detachedness may even be a hallmark of BPD (Young et al., 2003). Future research should further unravel the causes of negativistic thinking in BPD by including temperamentally matched controls, a comprehensive assessment of negative interpersonal childhood experiences, and a measure of dissociative or detached state. It further seems wise to keep in mind that this characteristic of BPD, like BPD itself, may have more developmental routes than one.

Although many studies have found negativistic evaluations in BPD, it is interesting that one carefully designed study (Ten Haaf \& Arntz, 2009) did find multidimensional dichotomous evaluations, but not negativistic evaluations in BPD. Ten Haaf and Arntz (2009) investigated interpersonal evaluations of patients with BPD in an ecologically possibly more valid situation, viz. in the context of a counseling session with pretended 'counselors-in-training' approaching the patients with a supportive, neutral or dejecting attitude. One explanation for the inconsistency is that patients with BPD may in some situations be capable of distancing or 'turning their schemas off' without becoming detached. Although some theorists claim that borderline patients lack distancing or 'mentalization' capacities (e.g., Fonagy, Target, Gergely, Allen, \& Bateman, 2003), this is not consequently supported by empirical research (e.g., Arntz et al., 2008a). In 
this case, the attention of the counselors and the privilege of judging them may have motivated the patients to judge them relatively objectively and the relative 'safety' of the situation (focus on the counselors instead of on themselves as well as a relatively clear task) may have enabled this. Borderline patients may also have been very uncomfortable with the task, possibly experiencing it as a reversed perpetrator-victim situation. Like individuals who were presented information which was threatening to their convictions demonstrated more complex arguments, borderline patients may also have over-elaborated their evaluations (see also, Bradley \& Westen, 2005). These over-elaborations may have the form of mixed positive and negative evaluations or 'multidimensional dichotomous evaluations'. This interpretation makes sense in the light of indications for decreases of multidimensional dichtomous evaluations in improving patients in a first therapy stage and reductions of negativistic evaluations in such patients in a subsequent therapy stage (see chapter 6). Better manipulation checks, such as physiological measures and extensive exit-interviews, may sort this out in future studies on the subject.

The findings on negativistic evaluations in BPD have major clinical implications. Negativistic thinking of borderline patients is very likely to cause problems in treatment. Forming a therapeutic alliance may be difficult because patients may expect to be harmed or shut themselves off by an armour of negativity. Keeping an unthreatening and supportive attitude and focusing on the development of mutual trust therefore seems crucial (see also, Spinhoven, Giesen-Bloo, van Dyck, Kooiman, \& Arntz, 2007). An initially positive alliance may further not be easily kept upright when for some reason the patient has begun to experience the therapy or the therapist as (potentially) abusive and the therapist reacts negatively. Exploration of this shift with knowledge that the patient tends to view others in a negative way, might then be helpful. Further, aiming at a reduction of negativistic thinking in BPD during treatment may not be necessary or doable, at least not initially. Our study even suggest that a decrease of (certain kinds of) negativistic thinking during a first stage of a change-oriented long-term therapy might be counterproductive (see chapter 6). These more negativistic evaluations may signify better access to schema's, which may be prerequisite for subsequent correction. Furthermore, a trend for a correlation between reductions of negativistic evaluations of negative schema-related persons and BPD-symptoms in a second stage of treatment (see chapter 6) suggests that it may only be needed or feasible to reduce trauma-related negativistic thinking. Future research has to sort out further how and which kind of negativistic 
thinking in BPD should optimally change during treatment.

\section{Some limitations and other methodological consider- ations}

The following section will discuss some limitations of the hypervigilance and extreme evaluations studies of this thesis, as well as some general methodological issues associated with doing experimental research with patients having BPD. The limitations sections will focus on the construct validity, i.e., the degree of correspondence of the assessed construct with the theoretical construct. In studies like those of this thesis, which aim to demonstrate hypothesized characteristics, this aspect of quality is crucial and more important than others, such as external validity and reliability. The degree in which potentially contaminating factors were controlled seems to be adequate. The three cross-sectional studies (chapters 2, 4, and 5) not only controlled for group differences such as age, sex and educational level, but also for presence of an axis-I and/or personality disorder. The longitudinal study comparing recovered and non-recovered patients (chapter 3) controlled for differences in severity of BPD symptoms and general anxiety at treatment start and (post-hoc) for differences in use of medication. The longitudinal study correlating decreases of BPD symptoms and reductions of extreme or negative evaluations (chapter 6) controlled for depression. The internal validity of the tasks in these studies, will be discussed in the following sections.

\section{Validity of hypervigilance assessment}

The studies of this thesis assessed hypervigilance in BPD with emotional Stroop tasks. This task had successfully shown effects in pilot research with BPD patients (Arntz et al., 2000) and many studies with other patients. The emotional Stroop task has received some criticism regarding its capacity to assess biases specifically in the attentional (and not response) phase of information processing (e.g., Dalgleish \& Watts, 1990; MacLeod, Mathews, \& Tata, 1986). However, this criticism is not undisputed. For example, Stroop-like inferences appeared to be unrelated to response biases (Luo, 1999). Another issue was, whether an emotional Stroop task assesses interference resulting from hypervigilance or the orienting of attention and not from other attentional processes, such as focused attention or delay in disengaging attention, though interferences caused 
by Stroop stimuli with very short presentation times ( $8-30 \mathrm{~ms}$; see chapter 2) do suggest that emotional Stroop task effects represent attentional orienting. Because these questions were (and still are) largely unanswered and a rudimentary assessment would suffice as well in this stage, we decided to stick to this task for our first follow-up studies. Future studies should however reconsider this choice now more detailed knowledge of psychopathology related attention and tasks tapping relevant attentional components has become available (e.g., see Mogg, Bradley, Miles, \& Dixon, 2004; Koster, Verschuere, Crombez, \& Van Damme, 2005).

The following measures and outcomes support the validity of our hypervigilance assessments (see also chapters 2 and 3). Internal validity of the emotional Stroop tasks was optimized by: (1) counterbalancing syllable lengths and presentation colors of the stimulus groups, (2) counterbalancing emotionality of schema-related and -unrelated stimuli (in task of chapter 2), and (3) randomizing presentation order and presentation color. Independent stimulus classifications and independent judgments of the emotionality of the stimuli added to the validity of the stimulus groups. Detection tasks checked whether the participants had not consciously perceived stimuli presented in the subliminal condition. With regard to the outcomes, the neutral and emotional word stimuli of both hypervigilance studies elicited the expected differential color-naming responses, viz. shorter and longer latencies respectively. Other investigators not always have found differential responses to emotional and neutral stimuli in BPD (e.g., comparable amygdala reactions to photographs of emotional and neutral facial expressions in Donegan et al., 2003). Maybe, the emotional Stroop task taps relatively early and clear biases, whereas tasks used by some other investigators assess later and more diffuse biases. The differential responses may alternatively also have resulted from different degrees of relatedness to relationships of the stimuli, as the neutral stimuli of our tasks were words associated with academic or business themes and/or house interiors. Furthermore, correlations of the emotional Stroop effects for negative stimuli and other anxiety related factors, such as anxiety symptoms and childhood trauma (see chapter 2) indicated a valid hypervigilance assessment.

The studies assessed schema-related information processing biases by the inclusion of schema-related and -unrelated stimuli in the emotional Stroop tasks. This resulted in differential response latencies to schema-related and -unrelated interpersonal stimuli in the BPD group in one of the two emotional Stroop studies (see chapter 2). As this study presented both emotionally nega- 
tive and positive stimuli and had a relatively long task, these characteristics may be necessary, to find schema-specific emotional Stroop effects in BPD. Effects of a primed emotional Stroop task, presenting objects (e.g., 'l') prior to the qualifiers (e.g., 'powerless') (see, Segal \& Gemar, 1997) may still be more robust and valid.

\section{Validity of extreme thinking assessment}

In this thesis, extreme thinking of borderline patients was assessed with evaluations of film characters (chapters 4 and 6), computer games and the participants themselves (chapter 5). The participants expressed their evaluations on several VASs. Scores for dichotomous thinking and splitting, respectively negativistic thinking were computed from the mean degree of extremity respectively negativity of these evaluations. Schema-relatedness of the extreme evaluations was operationalized by inclusion of schema related and unrelated stimuli and VASs. Outcomes for extreme thinking in BPD were not always consistent across the studies of this thesis and also differed in some respects from previous studies. Besides sample differences between the studies, characteristics of the assessments themselves may also explain some inconsistencies. This section will therefore elaborate on the main strengths and limitations of the way the studies of this thesis assessed extreme thinking in BPD, more specifically on the validity of the tasks, target stimuli, VASs and derived scores for the three types of extreme thinking.

Internal validity of the evaluation tasks was optimized by: (1) matching emotionality of schema-related and -unrelated stimuli, (2) counterbalancing presentation order of the stimulus categories, such as schema related and unrelated, and/or emotionally negative, positive and/or neutral stimuli, and (3) a (fixed) randomized order of the evaluation scales. Independent assessments corroborated the intended emotionality and schema specificity of the stimuli, with the exception of more negatively rated negative schema-related than negative schema-unrelated stimuli in one study of this thesis (see chapter 4).

The main strength of the evaluation task was the level of standardization of the stimuli and evaluations scales. The selected film characters, computer games in the studies of this thesis mainly differed on certain factors (emotional valence and/or schema-relatedness) and differed similarly on these factors across all groups. Many others evaluation studies have applied non-standardized ambiguous TAT pictures and own parents as stimuli and/or open for- 
mat evaluations. Standardization of the film characters could be increased further by removing irrelevant differences in appearance, language, gender, and style.

The main limitation of the evaluation task was its possibly somewhat restricted external validity. Evaluations of film characters may have been less dysfunctional as hypothesized because the patients may have been somewhat detached. Evaluations in a field setting (e.g., experience sampling paradigm) or in a more engaging situation in the laboratory (e.g., interactions with to-beevaluated others in role-plays) may have more external validity. The assessed evaluations of the borderline patients may, on the other hand, be more dysfunctional than the true evaluations because of a dramatic response style, which is assumed characteristic of BPD patients by some authors. Evaluation assessment with anchored scales or with implicit or indirect methods (e.g., assessment of implicit associations or of resultant behavior tendencies) may restrict these response biases. The assessment of extreme responses by means of predefined VASs has pros with regard to internal validity, but some cons regarding external validity, when compared to an open answer format. A structured closed response format is a standardized format, resulting in answers which are comparable over the participants in the sense that all participants judge the stimuli on the same dimensions. Predefined VASs may, on the other hand, not represent the usual or first evaluations of the participants; VASs may force the participant making evaluations on dimensions which they would not have considered automatically, possibly resulting in more strategic and more multidimensional evaluations.

The extreme thinking scores, particularly those for dichotomous thinking and splitting, should also be discussed here. Degree of dichotomous thinking was assessed with the means of the absolute evaluation scores on the VASs; splitting was computed by the absolute means of the (non-absolute) evaluations scores on the non-neutral VASs. One may argue that these calculations are improper because they also included moderately extreme evaluations. Although the contribution of these evaluations can be assumed to be relatively small, future studies should consider formulas based on extreme evaluations only (e.g., more extreme than some variance-based cut-off). An objection to the test of the applicability of splitting by comparing dichotomous thinking and splitting scores may be that the dichotomous thinking score can be smaller, but never larger, than the splitting score (Schwartz's proposition) resulting in a bias in favor of the multidimensionality hypothesis. However, as argued by Veen and 


\section{CHAPTER 7}

Arntz (2000), this is exactly what theories predicting splitting imply, i.e., all evaluations should be either good or bad. Furthermore, a significant bias towards dichotomous thinking resulting from mistaken ratings at the good pole despite engaging in all bad thinking is also very unlikely for the interpersonal evaluations studied in this thesis. Like in Veen and Arntz (2000), splitting scores were largely similar across the BPD and control groups, and dichotomous thinking scores were considerably higher than splitting scores (see chapters 4 and 5).

Schema-relatedness of the extreme evaluations was operationalized by inclusion of schema related and unrelated stimuli and VASs. However, the investigation of schemas in BPD, and especially the determinator of schemas that are specific to BPD, is still in its infancy: a reason for selecting our schemarelated interpersonal stimuli (i.e., the film characters of the studies in chapters 4 and 6) not exclusively on the basis of schema theories (for example of Pretzer, 1990) but also on other sources of information (such as observations of schema activation in BPD in interactions with partners, parents and other primary support group members; trauma models of BPD). Future investigations of schemas in BPD should not only study specific schema contents but also specific schema activation conditions, both in the patients themselves (e.g., low level of detachedness) and the situational context (e.g., low level of structure). For now, our findings are inevitably limited by the insufficient knowledge we still have about BPD schemas. Another issue with regard to the investigation of schema-relatedness of the evaluations is that the studies of this thesis did not differentiate evaluations on subtypes of schema-related traits (i.e., traits related to powerlessness, unacceptableness and malevolence). This may have obscured extreme evaluations for one subtype of these schema-related traits. For example, a recent study demonstrated aggresively instead of generally negatively biased evaluations of neutral characters in BPD (Barnow et al., 2009). Future studies should therefore apply more homogeneous schema-related scales.

Application of knowledge in the field of affect infusion into judgements (Forgas, 1995) may also further increase the validity of studies on extreme evaluations in BPD patients. Affect (or schema) infusion has been found to be minimal when a crystallized judgment is available (direct access processing) or when there is a motivation to counteract affect-infusion, such as mood-control or self-evaluation maintenance (motivated processing), whereas it appears to be maximal when judgments are primarily based on a how-do-l-feel-about-it heuristic (heuristic processing) or when people engage in on-line processing to observe and interpret ongoing stimuli (substantive processing). According to 
this analysis, experimental studies of the schema-relatedness of evaluation bias in patients with BPD should (1) include relatively unfamiliar and complex stimuli (e.g., unknown, non-stereotypic characters or games) to minimize direct access processing, (2) offer a non-judgmental trustworthy context to maximally eliminate motivational distortions (e.g., no evaluations of possibly future-therapists), (3) not make participants aware of their currently activated schemas in order to maximize heuristic processing (e.g., applying unobtrusive affect and schema measures), and (4) assess initial on-line judgments (e.g., intermittent evaluations) of preferably self-relevant stimuli to maximize substantive processing.

\section{Methodological aspects of experimental research with borderline patients}

Some specific characteristics of patients with BPD may unintentionally interact with investigations of these patients.

\section{Dysfunctional interpersonal schemas}

An experimental study is a more or less interpersonal situation in which the experimenter strives to control the participant to a certain extent. If the participant suffers from BPD these very aspects may trigger dysfunctional interpersonal schemas (e.g., 'I am powerless' and 'Others are malevolent') and counterschematic resistance before the experimental manipulation has even started. For example, a few BPD patients in our hypervigilance studies resisted the virtually impossible task of differentiating subliminally presented meaningful and nonsense words (detection task), possibly because of strong feelings of helplessness. As a consequence of this sensitivity, participants with BPD probably tend to experience a high level of evaluation apprehension, which may result in a social desirable, detached or hostile 'screw you' attitude (see Hoogstraten, 1979). In order to prevent such contaminating effects, experimenters carrying out experimental studies with borderline patients are recommended to create a study context in which participants feel respected, supported, and as much in control as the study goal allows. At best, the experimenter probes the procedure with a recovered or sub-syndromal borderline patient. In our studies we extensively informed the participants on the procedure ('several emotional film fragments will be presented'), tried to take away schema-congruent fears (e.g., 'there are no false and right answers'), gave as much control as possible ('you can decide to quit the experiment at any time and without explicit reason'), and 


\section{CHAPTER 7}

payed extra attention to the coaching of experimenters.

\section{Detachedness}

Clinical observations, patient reports, and studies of dissociative states in BPD (e.g., Stiglmayr et al., 2008) indicate that borderline patients often avoid experiencing emotions. The schema-mode model of Young et al. (2003) even assumes, this 'detached protector mode' is the usual mode of patients with BPD. A tendency to emotionally detachment in BPD may bias many study outcomes. For example, recent investigations have demonstrated that an unwillingness to experience emotional distress in order to pursue goals is more characteristic of BPD than deficient goal-directed behaviours (Gratz et al., 2006). Similarly, onedimensional evaluations in BPD may result from reduced motivation instead of defective capacities (see section on dichotomous thinking). Studies with BPD participants should therefore decrease detachedness (for example by asking participants to imagine and relate to the stimuli, exhausting the participants, intensifying the stimuli themselves), investigate participants in non-detached states (e.g., states of crisis), investigate phenomena which are more independent of detachedness or dissociation (for example implicit attention, evaluation and memory (e.g., see Huntjens, Peters, Woertman, van den Hart, \& Postma, 2007)), and/or control for detachedness (for example by the inclusion of emotionally neutral conditions, assessments of psychophysiological and other measures of dissociative state).

\section{Marked reactivity}

Patients with BPD can react with intense dysphoria, irritability and anxiety, as well as with impulsive acts ranging from mild self-damaging to suicidal behaviors (American Psychiatric Association, 2000). It is advisable to consider this marked reactivity with regard to the BPD participants but also the collaborators of the study. As delineated above, unintentional activation of dysfunctional interpersonal schemas and concurrent behaviors of the study participants may be prevented and tempered with a respectful, supportive, and control-allowing study context. Study collaborators, such as therapists and other members of the treatment teams, should however not be overlooked. Strong emotional and self-destructive reactions of patients with BPD, may make treatment teams apprehensive of experimental evocative studies with borderline patients. A premature ending of the research due to this apprehension may be prevented by: (1) 
a theoretically, methodologically, clinically and ethically sound research plan, (2) provision of clear information on the study to the whole treatment team, (3) follow-up visits to team meetings, and (4) an attentive and realistic attitude of the researcher.

\section{Closing remark}

For a long time, clinicians and researchers have assumed that patients with BPD are fundamentally different from other (neurotic) patients. The supposed inborn hyper-emotionality of borderline patients might be a more recent reformulation of this view. It has been said that the great enemy of truth is very often not the lie, but the myth. Is BPD still plagued by myths?

The studies of this thesis do put some doubts on the idea that BPD is fundamentally different. Borderline patients do not appear to function on a primitive cognitive-affective developmental level, they share important characteristics with (neurotic) anxiety disorder patients, and recovery from at least some fundamental problems such as hypervigilance seems possible. Maybe, BPD is not so different after all. Maybe, the emphasis has to shift from discontinuity to continuity, and from qualitative to quantitative differences. On the other side, clinicians are able to diagnose BPD surprisingly reliably. Does this only point to more precise diagnostic criteria, or also to essential differences between BPD and other disorders?

In short, the area of BPD still requires further studies investigating whether borderline patients are really so different than other patients, which particular characteristics make out the difference, and how this can be most effectively treated. This thesis is a contribution to this need. 


\section{Bibliography}

Ackerman, S. J., Clemence, A. J., Weatherill, R., \& Hilsenroth, M. J. (1999). Use of the TAT in the assessment of DSM-IV cluster B personality disorders. Journal of Personality Assessment, 73, 422-448.

Adler, G. (1985). Borderline psychopathology and its treatment. New York: Jason Aronson.

American Psychiatric Association. (1980). Diagnostic and statistical manual of mental disorders (Third ed.). Washington DC: American Psychiatric Association.

American Psychiatric Association. (1987). Diagnostic and statistical manual of mental disorders (Third, revised ed.). Washington DC: American Psychiatric Association.

American Psychiatric Association. (1994). Diagnostic and statistical manual of mental disorders (Fourth ed.). Washington DC: American Psychiatric Association.

American Psychiatric Association. (2000). Diagnostic and statistical manual of mental disorders (Fourth, text-revised ed.). Washington DC: American Psychiatric Association.

Andrulonis, P. A., Glueck, B. C., Stroebel, C. F., Vogel, N. G., Shapiro, A. L., \& Aldrigdge, D. M. (1981). Organic brain dysfunction and the borderline syndrome. The Psychiatric Clinics of North America, 4, 47-66.

Arntz, A. (1994). Treatment of borderline personality disorder: A challenge for cognitive-behavioural therapy. Behaviour Research and Therapy, 32, 419-430. 


\section{BIBLIOGRAPHY}

Arntz, A. (2004). Borderline personality disorder. In A. T. Beck, A. Freeman, D. D. Davis, \& Associates (Eds.), Cognitive therapy of personality disorders (2nd ed., pp. 187-215). New York: The Guilford Press.

Arntz, A., Appels, C., \& Sieswerda, S. (2000). Hypervigilance in borderline disorder: A test with the emotional Stroop paradigm. Journal of Personality Disorders, 14, 366-373.

Arntz, A., Bernstein, D., Oorschot, M., Robson, K., \& Schobre, P. (2008a). Theory of mind in borderline and cluster-C personality disorder. Submitted for publication.

Arntz, A., Dietzel, R., \& Dreessen, L. (1999). Assumptions in borderline personality disorder: Specificity, stability and relationship with etiological factors. Behaviour Research and Therapy, 37, 545-557.

Arntz, A., \& Dreessen, L. (1992). BPD-Klachtenlijst 47 (Borderline Personality Disorder Symptom Checklist). The Netherlands: Maastricht University.

Arntz, A., Dreessen, L., Schouten, E., \& Weertman, A. (2004). Beliefs in personality disorders: a test with the Personality Disorder Belief Questionnaire. Behaviour Research and Therapy, 42, 1215-1225.

Arntz, A., Gielen, D., van der Heijden, P., Cima, M., \& van der Veen, F. (2008b). Central and peripheral nervous system responses to emotional stimuli in borderline personality disorder: specificity and effects of psychotherapy. Submitted for publication.

Arntz, A., van den Hoorn, M., Cornelis, J., Verheul, R., van den Bosch, W. M. C., \& de Bie, A. J. H. T. (2003). Reliability and validity of the borderline personality disorder severity index. Journal of Personality Disorders, 17, 45-59.

Arntz, A., \& Veen, G. (2001). Evaluation of others by borderline patients. The Journal of Nervous and Mental Disease, 189, 513-521.

Baker, L., Silk, K. R., Westen, D., Nigg, J. T., \& Lohr, N. E. (1992). Malevolence, splitting, and parental ratings by borderlines. The Journal of Nervous and Mental Disease, 180, 258-264. 
Ball, J. R. (1999). A controlled evaluation of psychological treatments for anorexia nervosa. Dissertation Abstracts International: Section $B$ : The Sciences and Engineering, 59, 5781.

Barnow, S., Stopsack, M., Grab, H. J., Meinke, C., Spitzer, C., Kronmüller, K., \& Sieswerda, S. (2009). Interpersonal evaluation bias in borderline personality disorder. Behaviour Research and Therapy (2009), doi:10.1016/j.brat.2009.02.003.

Beck, A. T. (1976). Cognitive therapy and the emotional disorders. New York: International Universities Press.

Beck, A. T. (1979). Cognitive therapy of depression. New York: The Guilford Press.

Beck, A. T. (1996). Beyond belief: A theory of modes, personality, and psychopathology. In P. M. Salkovskis (Ed.), Frontiers of cognitive therapy (pp. 1-47). New York: The Guilford Press.

Benjamin, L. S., \& Wonderlich, S. A. (1994). Social perceptions and borderline personality disorder: The relation to mood disorders. Journal of Abnormal Psychology, 103, 610-624.

Bille, A., \& Bjalkeskog, L. (1992). The best intentions [Motion Picture]. Sweden: Swedish Television.

Blatt, S. J., \& Auerbach, J. S. (1988). Normal development and psychopathological impairment of the concept of the object on the rorschach. Journal of Personality Disorders, 2, 198-211.

Blatt, S. J., Brenneis, B., Schimek, J. G., \& Glick, M. (1976). Normal development and psychopathological impairment of the concept of the object on the rorschach. Journal of Abnormal Psychology, 85, 364-373.

Bonis, M., De Boeck, P., Lida-Pulik, H., Hourtané, M., \& Féline, A. (1998). Selfconcept and mood: A comparative study between depressed patients with and without borderline personality disorder. Journal of Affective Disorders, 48, 191-197.

Bradley, B. P., Mogg, K., White, J., \& Millar, N. (1995). Selective processing of negative information: effect of clinical anciety, concurrent depression, and awareness. Journal of Abnormal Psychology, 104, 532-536. 


\section{BIBLIOGRAPHY}

Bradley, R., \& Westen, D. (2005). The psychodynamics of borderline personality disorder: A view from developmental psychopathology. Development and Psychopathology, 17, 927-957.

Bürström, K., Johannesson, M., \& Diderichsen, F. (2001). Swedish population health-related quality of life results using the eq-5d. Quality of Life Research, 10, 621-635.

Carter, F. A., Bulik, C. M., McIntosh, V. V., \& Joyce, P. R. (2000). Changes on the Stroop test following treatment: Relation to word type, treatment condition, and treatment outcome among women with bulimia nervosa. International Journal of Eating Disorders, 28, 349-355.

Chechik, J. S., Arnold, S., \& Roth, D. (1993). Benny and Joon [Motion Picture]. United States: Metro-Goldwyn-Mayer.

Clarkin, J. F., Yeomans, F. E., \& Kernberg, O. F. (1999). Psychotherapy for borderline personality. New York: John Wiley \& Sons.

Coccaro, E. F. (1989). Central serotonin and impulsive agression. British Journal of Psychiatry, 155 (suppl. 8), 52-62.

Cohen, J. B. (1988). Statistical power analysis for the behavioural sciences (Second ed.). Hillsdale: Lawrence Erlbaum.

Cooper, M. J., \& Fairburn, C. G. (1994). Changes in selective information processing with three psychological treatments for bulimia nervosa. British Journal of Clinical Psychology, 33, 353-356.

Côté, S., \& Bouchard, S. (2005). Exposure with psychophysiological and information processing measures. Applied psychophysiology and Biofeedback, 30, 217-232.

Dalgleish, T., \& Watts, F. N. (1990). Biases of attention and memory in disorders of anxiety and depression. Clinical Psychology Review, 10, 589-604.

Damen, K. F. M., de Jong, C. A. J., \& van der Kroft, P. J. A. (2004). Interrater reliability of the structured interview for dsm-iv personality in an opioiddependent patient sample. European Addiction Research, 10, 99-104.

Darabont, F., \& Valdes, D. (1999). The green mile [Motion Picture]. United States: Castle Rock Entertainment and Warner Brothers. 
de Bont, J., Kennedy, K., Bryce, I., \& Crichton, M. (1996). Twister [Motion Picture]. United States: Warner Brothers and Universal Pictures.

Devineni, T., Blanchard, E. B., Hickling, E. J., \& Buckley, T. C. (2004). Effect of psychological treatment on cognitive bias in motor vehicle accidentrelated posttraumatic stress disorder. Anxiety Disorders, 18, 211-231.

Donegan, N. H., Sanislow, C. A., Blumberg, H. P., Fulbright, R. K., Lacadie, C., Skudlarski, P., Gore, J. C., Olson, I. R., McGlashan, T. H., \& Wexler, B. E. (2003). Amygdala hyperreactivity in borderline personality disorder: Implications for emotional dysregulation. Biological Psychiatry, 54, 12841293.

Downey, G., \& Walker, E. (1989). Social cognition and adjustment in children at risk for psychopathology. Developmental Psychology, 25, 835-845.

Dreessen, L., \& Arntz, A. (1995). The Personality Disorder Belief Questionnaire $(P D B Q)$ (short version). Maastricht: Author.

Dritschel, B. H., \& Teasdale, J. D. (1991). Individual differences in affect-related cognitive operations elicited by experimental stimuli. British Journal of Clinical Psychology, 30, 151-160.

First, M. B., Spitzer, R. L., Gibbon, M., \& Williams, J. B. W. (1997). Structured clinical interview for DSM-IV axis I disorders (SCID-I) [interview]. New York: New York State Psychiatric Institute.

First, M. B., Spitzer, R. L., Gibbon, M., Williams, J. B. W., \& Benjamin, L. (1994). Structured clinical interview for DSM-IV axis I/ personality disorders (SCID-II) [interview]. New York: New York State Psychiatric Institute.

Foa, E. B., \& McNally, R. J. (1986). Sensitivity to the feared stimuli in obsessivecompulsives: A dichotic listening analysis. Cognitive Therapy and Research, 10, 477-485.

Foa, E. B., Steketee, G., \& Rothbaum, B. O. (1989). Behavioral/cognitive conceptualizations of post-traumatic stress disorder. Behaviour Therapy, 20, 155-176.

Fonagy, P., Target, M., \& Gergely, G. (2000). Attachment and borderline personality disorder. The Psychiatric Clinics of North America, 23, 103-122. 


\section{BIBLIOGRAPHY}

Fonagy, P., Target, M., Gergely, G., Allen, J. G., \& Bateman, A. W. (2003). The developmental roots of borderline personality disorder in early attachment relationships: A theory and some evidence. Psychoanalytic Inquiry, 23, 412-459.

Forgas, J. P. (1995). Mood and judgment: the affect infusion model (aim). Psychological Bulletin, 117, 39-66.

Fossati, A., Maddeddu, F., \& Maffei, C. (1999). Borderline personality disorder and childhood sexual abuse: a meta-analytic study. Journal of Personality Disorders, 13, 268-280.

Gabbard, G. O. (1994). Psychodynamic psychiatry in clinical practice: the DSM IV. Washington DC: American Psychiatric Press.

Giesen-Bloo, J., Arntz, A., \& Schouten, E. (2006a). The Borderline Personality Disorder Checklist: Psychometric evaluation and factorial structure in clinical and nonclinical samples. Submitted for publication.

Giesen-Bloo, J., van Dyck, R., Spinhoven, P., van Tilburg, W., Dirksen, C., van Asselt, T., Kremers, I., Nadort, M., \& Arntz, A. (2006b). Outpatient psychotherapy for borderline personality disorder: Randomized trial of schema-focused therapy versus transference-focused psychotherapy. Archives of General Psychiatry, 63, 649-658.

Giesen-Bloo, J., Wachters, L., Schouten, E., \& Arntz, A. (2006c). Assessment of borderline personality disorder with the Borderline Personality Disorder Severity Index - IV: Psychometric evaluation and dimensional structure. Submitted for publication.

Gratz, K. L., Rosenthal, M. Z., Tull, M. T., Lejuez, C. W., \& Gunderson, J. G. (2006). An experimental investigation of emotion dysregulation in borderline personality disorder. Journal of Abnormal Psychology, 115, 850-855.

Groenestijn, M. A. C., Akkerhuis, G. W., Kupka, R. W., Schneider, N., \& Nolen, W. A. (1999). Gestructureerd klinisch interview voor de vaststelling van $D S M-I V$ as-I stoornissen (SCID-I). (Dutch version of the structured clinical interview for DSM-IV axis I disorders (SCID-I).). Lisse, Netherlands: Swets Test Publishers. 
Gunderson, J. G., \& Sabo, A. N. (1993). The phenomenological and conceptual interface between borderline personality disorder and PTSD. American Journal of Psychiatry, 150, 19-27.

Haines, R., Rappaport, M., \& Goldberg, L. J. (1984). Something about Amelia [Motion Picture]. United States: ABC Theatre.

Harter, S. (1986). Cogntive-developmental processes in the integration of concepts about emotions and the self. Social Cognition, 4, 119-151.

Harvey, A. G., Watkins, E., Mansell, W., \& Shafran, R. (2004). Cognitive behavioural processes across psychological disorders. New York: Oxford University Press.

Herman, J. L. (1992). Trauma and recovery. New York: Basic Books.

Herman, J. L., Perry, J. C., \& van der Kolk, B. A. (1989). Childhood trauma in borderline personality disorder. American Journal of Psychiatry, 146, $490-495$.

Hermans, D. (1994). De 'Zelf-Beoordelings-Vragenlijst' (ZBV). Gedragstherapie, 27, 145-148.

Herpertz, S. C., Dietrich, T. M., Wenning, B., Krings, T., Erberich, S. G., Wilmes, K., Thron, A., \& Sass, H. (2001a). Evidence of abnormal amygdala functioning in borderline personality disorder. Biological Psychiatry, 50, 292-298.

Herpertz, S. C., Schwenger, U. B., Kunert, H. J., Lukas, G., Gretzer, U., Nutzmann, J., Schuerkens, A., \& Sass, H. (2000). Emotional responses in patients with borderline as compared with avoidant personality disorder. Journal of Personality Disorders, 14, 339-351.

Herpertz, S. C., Werth, U., Lukas, G., Qunaibi, M., Schuerkens, A., Kunert, H., Freese, R., Flesch, M., Mueller-Isberner, R., Osterheider, M., \& Sass, H. (2001b). Emotion in criminal offenders with psychopathy and borderline personality disorder. Archives of General Psychiatry, 58, 737-745.

Hoch, P. H., \& Cattell, J. P. (1959). The diagnosis of pseudoneurotic schizophrenia. The Psychiatric quarterly, 33, 17-43. 


\section{BIBLIOGRAPHY}

Hoogstraten, J. (1979). De machteloze onderzoeker. voetangels en klemmen van sociaal-wetenschappelijk onderzoek. (the powerless scientist. pitfalls of social-scientific research). Amsterdam: Boom.

Horowitz, M. J. (1993). Stress-response syndromes. a review of posttraumatic stress and adjustment disorders. In J. P. Wilson \& B. Raphael (Eds.), International handbook of traumatic stress syndromes (pp. 49-59). New York \& London: Plenum Press.

Huntjens, R. J. C., Peters, M. L., Woertman, L., van den Hart, O., \& Postma, A. (2007). Memory transfer for emotionally valenced words between identities in dissociative identity disorder. Behaviour Research and Therapy, $45,775-789$.

Ingenhoven, T., \& van den Brink, W. (1994). Borderline persoonlijkheidsstoornis: diagnostische en epidemiologische aspecten. In J. J. L. Derksen \& H. Groen (Eds.), Handboek voor de behandeling van borderline patiënten (pp. 28-42). Utrecht: De Tijdstroom.

Jaffe, S., Lansing, S., \& Lyne, A. (1987). Fatal attraction [Motion Picture]. United States: Paramount Studio.

Janoff-Bulman, R. (1991). Assumptive worlds and the stress of traumatic events: Applications of the scheme construct. Social Cognition, 7, 113136.

Johnsen, B. H., Laberg, J. C., Cox, W. M., Vaksdal, A., \& Hugdahl, K. (1994). Alcoholic subjects' attentional bias in the processing of alcohol-related words. Psychology of Addictive Behaviors, 8, 111-115.

Jong de, C. A. J., Derks, F. C. H., van Oel, C. J., \& Rinne, T. (1996). Gestructureerd interview voor de DSM-IV persoonlijkheidsstoornissen (SIDP-IV) [interview (in Dutch)]. NOVADIC publicatie.

Joseph, S., Williams, R., \& Yule, W. (1997). Understanding post-traumatic stress. A psychosocial perspective on ptsd and treatment. Chichester, England: John Wiley and Sons.

Kampman, M., Keijsers, G. P. J., Verbraak, M. J. P. M., Näring, G., \& Hoogduin, C. A. L. (2002). The emotional Stroop: A comparison of panic disorder patients, obsessive-compulsive patients, and normal controls, in two experiments. Anxiety Disorders, 16, 425-441. 
Kernberg, O. (1966). Structural derivatives of object relationships. The International Journal of Psychoanalysis, 47, 236-253.

Kernberg, O. (1967). Borderline personality organization. Journal of the American Psychoanalytic Association, 15, 641-685.

Kernberg, O. (1975). Borderline conditions and pathological narcissism. New York: Jason Aronson.

Kernberg, O. (1996). A psychoanalytic theory of personality disorders. In J. F. Clarkin \& M. F. Lenzenweger (Eds.), Major theories of personality disorder (pp. 106-140). New York: The Guildford Press.

Kindt, M., \& Van den Hout, M. (2001). Selective attention and anxiety: a perspective on developmental issues and the causal status. Journal of Psychopathology and Behavioural Assessment, 23, 193-202.

Kosminsky, P. (1997). No child of mine [TV Drama]. England: Meridian TV.

Koster, E. H. W., Verschuere, B., Crombez, G., \& Van Damme, S. (2005). Timecourse of attention for threatening pictures in high and low trait anxiety. Behaviour Research and Therapy, 43, 1087-1098.

Kremers, I. P., Spinhoven, P., \& Van der Does, A. J. W. (2004). Autobiographical memory in depressed and non-depressed patients with borderline personality disorder. British Journal of Clinical Psychology, 43, 17-29.

Kroll, J. (1988). The challenge of the borderline patient. Competency in diagnosis and treatment. New York: W. W. Norton \& Company.

Kurtz, J. E., \& Morey, L. C. (1998). Negativism in evaluative judgments of words among depressed outpatients with borderline personality disorder. Journal of Personality Disorders, 12, 351-361.

Larsen, R. J., Diener, E., \& Cropanzano, R. S. (1987). Cognitive operations associated with individual differences in affect intensity. Journal of Personality and Social Psychology, 53, 767-774.

Lavy, E. H., \& van den Hout, M. A. (1993). Selective attention evidenced by pictorial and linguistic stroop tasks. Behavior Therapy, 24, 645-657. 


\section{BIBLIOGRAPHY}

Lavy, E. H., \& van den Hout, M. A. (1994). Cognitive avoidance and attentional bias: Causal relationships. Cognitive Therapy and Research, 18, 179191.

Lavy, E. H., van den Hout, M. A., \& Arntz, A. (1993). Attentional bias and spider phobia: Conceptual and clinical issues. Behaviour Research and Therapy, 31, 17-24.

Layden, M. A., Newman, C. F., Freeman, A., \& Morse, S. (1993). Cognitive therapy of borderline personality disorder. Needham Heights, New York: Allyn and Bacon.

Leichsenring, F. (1990). Discriminating borderline from neurotic patients. Psychopathology, 23, 21-26.

Leichsenring, F. (1991). Discriminating schizophrenics from borderline patients: Study with the Holtzman inktblot technique. Psychopathology, 24, 225231.

Leichsenring, F. (1999). Splitting: An empirical study. Bulletin of the Menninger Clinic, 63, 520-537.

Leichsenring, F., Roth, T., \& Meyer, H. A. (1992). Kognitiver Stil bei Borderline - im Vergleich zu neurotischen Patienten: Ambiguitäts-Vermeidung und verminderte Abstraktheit (Cognitive style in borderline patients compared with neurotic patients: avoidance of ambiguity and reduced abstractness). Diagnostica, 38, 52-65.

Lerner, H. D., \& St. Peter, S. (1984). Patterns of object rlations in neurotic, borderline and schizophrenic patients. Psychiatry, 47, 77-92.

Levine, D., Marziali, E., \& Hood, J. (1997). Emotion processing in borderline personality disorders. The Journal of Nervous and Mental Disease, 185, 240-246.

Linehan, M. M. (1993). Cognitive-behavioral treatment of borderline personality disorder. New York: The Guilford Press.

Lobbestael, J., \& Arntz, A. (2008). Emotional, cognitive and physiological correlates of abuse-related stress in borderline and antisocial personality disorder. Submitted for publication. 
Lobbestael, J., Arntz, A., \& Sieswerda, S. (2005). Schema modes and childhood abuse in borderline and antisocial personality disorders. Journal of Behavior Therapy and Experimental Psychiatry, 36, 204-263.

Loranger, A. W., Sartorius, N., Andreoli, A., Berger, P., Buchheim, P., Channabasavanna, S. M., Coid, B., Dahl, A., Diekstra, R. F. W., Ferguson, B., Jacobsberg, L. B., Mombour, W., Pull, C., Ono, Y., \& Regier, D. A. (1994). The international personality disorder examination. the world health organization / alcohol, drug abuse, and mental health administration international pilot study of personality disorders. Archives of General Psychiatry, 51, 215-224.

Lundh, L. G., \& Öst, L. G. (2001). Attentional bias, self-consciousness, and perfectionism in social phobia. Scandinavian Journal of Behaviour Therapy, 30, 4-16.

Lundh, L. G., Wikstrom, J., Westerlund, J., \& Öst, L. G. (1999). Preattentive bias for emotional information in panic disorder with agoraphobia. Journal of Abnormal Psychology, 108, 222-232.

Luo, C. R. (1999). Semantic competition as the basis of Stroop interference. Psychological Science, 10, 35-40.

Lupke, U., \& Ehlert, U. (1998). Selective Aufmerksamkeitslenkung auf gesundheitsbedrohliche Reize bei Patienten mit einer somatoformen Störung (Attentional bias for health threat related cues in patients with somatoform disorders). Zeitschrift für Klinische Psychologie, 27, 163-171.

Lynch, T. R., Rosenthal, M. Z., Kosson, D. S., Cheavens, J. S., Lejuez, C. W., \& Blair, R. J. R. (2006). Heightened sensitivity to facial expressions of emotion in borderline personality disorder. Emotion, 6, 647-655.

MacLeod, C. M. (1991). Half a century of research on the stroop effect: An integrative review. Psychological Bulletin, 109, 163-203.

MacLeod, C. M., \& Hagan, R. (1992). Individual differences in the selective processing of threatening information, and emotional responses to a stressful life event. Behaviour Research and Therapy, 30, 151-161.

MacLeod, C. M., Mathews, A., \& Tata, P. (1986). Attentional bias in emotional disorders. Journal of Abnormal Psychology, 95, 15-20. 


\section{BIBLIOGRAPHY}

MacLeod, C. M., Rutherford, E., Campbell, L., Ebsworthy, G., \& Holker, L. (2002). Selective attention and emotional vulnerability: Assessing the causal basis of their association through the experimental manipulation of attentional bias. Journal of Abnormal Psychology, 111, 107-123.

Maffei, C., Fossati, A., Agostoni, I., Barraco, A., Bagnato, M., Deborah, D., Namia, C., Novella, L., \& Petrachi, M. (1997). Interrater reliability and internal consistency of the Structured Clinical Interview for DSM-IV Axis II Personality Disorders (SCID-II), version 2.0. Journal of Personality Disorders, 11, 279-284.

Mathews, A., Mogg, K., Kenthish, J., \& Eysenck, M. (1995). Effect of psychological treatment on cognitive bias in generalized anxiety disorder. Behaviour Research and Therapy, 33, 293-303.

Mattia, J. I., Heimberg, R. G., \& Hope, D. A. (1993). The revised Stroop colornaming task in social phobics. Behaviour Research and Therapy, 31, 305-313.

McNally, R. J. (1998). Experimental approaches to cognitive abnormality in posttraumatic stress disorder. Clinical Psychology Review, 18, 971-982.

McNally, R. J., Riemann, B. C., \& Kim, E. (1990). Selective processing of threat cues in panic disorder. Behaviour Research and Therapy, 28, 407-412.

Melito, R. (1983). Cogntive aspects of splitting and libidinal object constancy. Journal of the American Psychoanalytic Association, 31, 515-534.

Miller, G. A., \& Chapman, J. P. (2001). Misunderstanding analysis of covariance. Journal of Abnormal Psychology, 110, 40-48.

Mogg, K., Bradley, B. P., Hyare, H., \& Lee, S. (1998). Selective attention to foodrelated stimuli in hunger: are attentional biases specific to emotional and psychpathological states, or are they also found in normal drive states? Behaviour Research and Therapy, 36, 227-237.

Mogg, K., Bradley, B. P., Miles, F., \& Dixon, R. (2004). Time course of attentional bias for threat scenes: Testing the vigilance-avoidance hypothesis. Cognition and Emotion, 18, 689-700. 
Mogg, K., Bradley, B. P., Millar, N., \& White, J. (1995). A follow-up study of cogntive bias in generalized anxiety disorder. Behaviour Research and Therapy, 33, 927-935.

Mogg, K., Kentish, J., \& Bradley, B. (1993). Effects of anxiety disorder and awareness onn colour-identification latencies for emotional words. Behaviour Research and Therapy, 31, 559-567.

Nigg, J. T., Lohr, N. E., Westen, D., Gold, L. J., \& Silk, K. R. (1992). Malevolent object representations in borderline personality disorder and major depression. Journal of Abnormal Psychology, 101, 61-67.

Nigg, J. T., Silk, K. R., Westen, D., Lohr, N. E., Gold, L. J., Goodrich, S., \& Ogata, S. (1991). Object representations in the early memories of sexually abused borderline patients. American Journal of Psychiatry.

Ornduff, S. R. (2000). Childhood maltreatment and malevolence: quantitative research findings. Clinical Psychology Review, 20, 997-1018.

Paris, J. (1993). The treatment of borderline personality disorder in light of research on its long term outcome. Canadian Journal of Psychiatry, 38, S28-S34.

Paris, J. (2004). Gender differences in personality traits and disorders. Current Psychiatry Reports, 6, 71-74.

Pfohl, B., Blum, N., \& Zimmerman, M. (1996). Structured interview for DSM-IV personality disorders (SIDP-IV). Unpublished manual, University of lowa College of Medicine, lowa City.

Plummer, L. S., \& Das, S. S. (1973). A study of dichotomous thought processes in accident-prone drivers. British Journal of Psychiatry, 122, 289-294.

Polanski, R. (1992). Bitter moon [Motion Picture]. United States: Columbia Tristar Studios.

Posner, M. I., Rothbart, M. K., Vizueta, N., Levy, K. N., Evans, D. E., Thomas, K. M., \& Clarkin, J. F. (2002). Attentional mechanisms of borderline personality disorder. Proceedings of the National Academy of Sciences of the United States of America, 99, 16366-16370. 
BIBLIOGRAPHY

Pretzer, J. (1990). Borderline personality disorder. In T. A. Beck, A. Freeman, \& Associates (Eds.), Cognitive therapy of personality disorders (pp. 176207). New York: The Guilford Press.

Radford, M., Cecchi Gori, M., Cecchi Gori, V., \& Daniele, G. (1994). I/ postino [Motion Picture]. Italy: Miramax.

Relyea, R., \& Sagal, B. (1978). The awakening land [Motion Picture]. United States: Warner Bros.

Renneberg, B., Heyn, K., Gebhard, R., \& Bachmann, S. (2005). Facial expression of emotions in borderline personality disorder and depression. Journal of Behavior Therapy and Experimental Psychiatry, 36, 183-196.

Renneberg, B., Stäbler, K., Fiedler, P., Weisbrod, M., \& Kaiser, S. (2008). It's difficult to smile: Enduring problems with the expression of positive emotions in borderline personality disorder. Submitted for publication.

Roeg, N., \& Katz, P. (1973). Don't look now [Motion Picture]. United Kingdom: British Lion, Casey Productions Ltd., and Eldorado Films.

Rohde-Dachser, C. (1986). Borderlinestörungen. In K. P. Kisker, H. Lauter, J. E. Meyer, C. Müller, \& E. Strömgren (Eds.), Psychiatrie der Gegenwart 1. Neurosen, psychosomatische Erkrankungen, Psychotherapie (pp. 125150). Berlin: Springer Verlag.

Rosenthal, M. Z., Cheavans, J. S., Lejuez, C. W., \& Lynch, T. R. (2005). Thought suppression mediates the relationship between negative affect and borderline personality disorder symptoms. Behaviour Research and Therapy, 43, 1173-1185.

Sable, P. (1997). Attachment, detachment and borderline personality disorder. Psychotherapy: Theory, Research, Practice, Training, 34, 171-181.

Sabo, A. N. (1997). Etiological significance of associations between childhood trauma and borderline personality disorder: conceptual and clinical implications. Journal of Personality Disorders, 11, 50-70.

Samuels, J., Eaton, W. W., Bienvenu, O. J., Brown, C. H., Costa, P. T., \& Nestadt, G. (2002). Prevalence and correlates of personality disorders in a community sample. British Journal of Psychiatry, 180, 536-542. 
Schmahl, C. G., Elzinga, B. M., Vermetten, E., Sanislow, C., McGlashan, T. H., \& Bremner, J. D. (2003). Neural correlates of memories of abandonment in women with and without borderline personality disorder. Society of Biological Psychiatry, 54, 142-151.

Schnell, K., \& Herpertz, S. C. (2007). Effects of dialectic-behavioral-therapy on the neural correlates of affective hyperarousal in borderline personality disorder. Journal of Psychiatric Research, 41, 837-847.

Scott, J., \& Hicks, S. (1996). Shine [Motion Picture]. Australia: Momentum Films.

Segal, H. G., Westen, D., Lohr, N. E., Silk, K. R., \& Cohen, R. (1992). Assessing object relations and social cognition in borderline personality disorders from stories told to the picture arrangement subtest of the WAIS-R. Journal of Personality Disorders, 6, 458-470.

Segal, Z. V., \& Gemar, M. (1997). Changes in cognitive behaviour therapy for depression: a primed Stroop study. Cognition and Emotion, 11, 501-516.

Sheffield, M. W., Barlow, S. H., Lambert, M. J., Hoyal, H., Thompson, K. C., \& Garbutt, M. (1999). A qualitive examination of borderline personality disordered (BPD) patients: interpersonal dynamics and underlying paradoxes. Journal of Personality Disorders, 13, 287-296.

Shin, L., Whalen, P. J., Pitman, R. K., Bush, G., Macklin, M. L., Lasko, N. B., Orr, S. P., Mclnerney, S. C., \& Rauch, S. L. (2001). An fMRI study of anterior cingulate function in posttraumatic stress disorder. Society of Biological Psychiatry, 50, 932-942.

Siever, L. J., \& Davis, K. L. (1991). A psychobiological perspective on the personality disorder. American Journal of Psychiatry, 148, 1647-1658.

Simpson, E. B., Yen, S., Costello, E., Rosen, K., Begin, A., Pistorello, J., \& Pearlstein, T. (2004). Combined dialectical behavior therapy and fluoxetine in the treatment of borderline personality disorder. Journal of Clinical Psychiatry, 65, 379-385.

Spear, W. E., \& Sugarman, A. (1984). Dimensions of internalized object relations in borderline and schizophrenic patients. Psychoanalytic psychology, 1, 113-129. 


\section{BIBLIOGPAPHY}

Spielberger. C. D. Gorsuch. R. L. Lusthene. R. E. (1970). Manual for the State-Trat Anxiety Inventory \& STAll. Palo Alto. CA: Consulting Psychologists Press

Spinhoven. P.. Gesen-Bloo. J. van Dyck. R. Kooiman. K., \& Arntz. A. (2007). The therapeutic alliance in schema-focused therapy and transferencefocused psychotherapy for borderline personality disorder. A Journal of Consulting and Clinical Psychology. 75, 104-115

Spitzer. R. L., First. M. B. Gibbon. M. \& Williams, J. B. W. (1995). Structured clinical interview for DSM.IV personality disorders [interview]. New York: New York State Psychiatric Institute.

Stein, K. F. (1996). Affect instability in adults with a borderline personality disorder. Archives of Psychiatric Nursing, 10, 32-40.

Steinberg. M. (1993). Structured clinical interview for DSM-IV dissociative disorders (SCID-D) [interview]. Washington, DC: American Psychiatric Association.

Stern, A. (1938). Psychoanalytic investigation of and therapy in the borderline group of neuroses. The Psychoanalytic quarterly, 7, 467-489.

Stern, M. I. Herron, W. G. Primavera, L. H. \& Kakuma, T. (1997). Interpersonal perceptions of depressed and borderline inpatients. Journal of Clinical Psychology, 53, 41-49.

Stiglmayr, C., Ebner-Priemer, U. W., Bretz, J., Behm, R., Mohse, M., Lammers, C. H., Anghelescu, I. G., Schmahl, C., Schlotz, W., Kleindienst, N., \& Bohus, M. (2008). Dissociative symptoms are positively related to stress in borderline personality disorder. Acta Psychiatrica Scandinavica, 117, $139-147$.

Stiglmayr, C., Shapiro, D. A., Stieglitz, R. D., Limberger, M. F., \& Bohus, M. (2001). Experience of aversive tension and dissociation in female patients with borderline personality disorder: A controlled study. Journal of Psychiatric Research, 35, 111-118.

Stuart, J., Westen, D., Lohr, N., Benjamin, J., Becker, S., Vorus, N., \& Silk, K. (1990). Object relations in borderlines, depressives, and normals: An examination of human responses on the rorschach. Journal of Personality Assessment, 55, 296-318. 
Ten Haaf, J. E., \& Arntz, A. (2009). Cognitive characteristics of judgments of borderline patients in social interaction. Submitted for publication.

Tisch, S., \& Nelson, J. (1994). Corrina, Corrina [Motion Picture]. Unites States: Jesse Nelson Films.

Torgersen, S., Kringlen, E., \& Cramer, V. (2001). The prevalence of personality disorders in a community sample. Archives of General Psychiatry, 58, 590-596.

Trull, T. J. (2001). Structural relations between borderline personality disorder features and putative etiological correlates. Journal of Abnormal Psychology, 110, 471-481.

van Asselt, A. D. I., Dirksen, C., \& Arntz, A. (2008). Outpatient psychotherapy for borderline personality disorder: Cost-effectiveness of schema-focused therapy versus transference-focused psychotherapy. British Journal of Psychiatry, 192, 450-457.

van den Bossche, C., Kremers, I., Sieswerda, S., \& Arntz, A. (1999). Vragenlijst naar Belastende Gebeurtenissen (VBG)(Structured Childhood Trauma Interview). Unpublished.

van den Hout, M. A., Tenney, N., Huygens, K., \& de Jong, P. (1997). Preconscious processing bias in specific phobia. Behaviour Research and Therapy, 35, 29-34.

van den Hout, M. A., Tenney, N., Huygens, K., \& Merckelbach, H. (1995). Responding to subliminal threat cues is related to trait anxiety and emotional vulnerability: a successful replication of MacLeod \& Hagan (1992). Behaviour Research and Therapy, 33, 451-454.

van der Ploeg, H. M. (1980). Validiteit van de Zelf-Beoordelings-Vragenlijst. (validity of the Zelf-Beoordelings-Vragenlijst). Nederlands Tijdschrift voor de Psychologie en haar Grensgebieden, 35, 243-249.

Veen, G., \& Arntz, A. (2000). Multidimensional dichotomous thinking characterizes borderline personality disorder. Cognitive Therapy and Research, $24,23-45$. 


\section{BIBLIOGRAPHY}

Ventura, J.. Liberman. R. P. Green. M. F. Shaner. A.. \& Mintz. J. (1998). Training and quality assurance with Structured Clinical Intervew for DSMIV ISCID-IP) Psychiatry Research, 79. 163-173

Verhaak. C. M. Smeenk, J. M. J. van Minnen. A., \& Kraaimaat. F. W. (2004), Neuroticism, preattentive and attentional biases towards threat. and anxety before and after a severe stressor: a prospective study. Personality and Indwidual Differences. $36.767-778$

Verhoeven. P. \& de Houwer, R. (1973). Turks fruit [Motion Picture] the Netherlands: Arcade.

Wagner, A W. \& Linehan, M. M. (1999). Facial expression recognition ability among women with borderline personality disorder: Implications for emotion regulation? Journal of Personality Disorders, 13, 329-344.

Waller, G. \& Button. J. (1999). Processing of threat cues in borderline personality disorder. Unpublished manuscript, University of Southampton, Southampton, United Kingdom

Watts, F. N., McKenna, F. P, Sharrock, R., \& Trezise, L. (1986). Colour naming of phobia-related words. The British Journal of Psychology, 77, 97-108.

Weertman, A., Arntz, A.. Dreessen, L., Velzen, C. van, \& Vertommen, S. (2003). Short-interval test-retest interrater reliability of the Dutch version of the structured clinical interview for DSM-IV personality disorders (SCID-II). Journal of Personality Disorders, 17, 562-567.

Weertman, A., Arntz, A., \& Kerkhofs, M. L. M. (2000). Gestructureerd klinisch interview voor DSM-IV as-II persoonlijkheidsstoornissen (SCID-II). (Dutch version of the structured clinical interview for DSM-IV axis II personality disorders (SCID-II).) [interview]. Lisse, Netherlands: Swets \& Zeitlinger.

Westen. D. (1990). Towards a revised theory of borderline object relations: contributions of empirical research. The International Journal of Psychoanalysis, 71, 661-693.

Westen, D., Lohr, N., Silk, K. R., Gold, L., \& Kerber, K. (1990a). Object relations and social cognition in borderlines, major depressives, and normals: $A$ Thematic Apperception Test analysis. A Journal of Consulting and Clinical Psychology, 2, 355-364. 
Westen, D., Ludolph, P., Block, J., Wixom, J., \& Wiss, F. C. (1990b). Developmental history and object relations in psychiatrically disturbed adolescent girls. American Journal of Psychiatry, 147, 1061-1068.

Westen, D., Ludolph, P., Lerner, H., Ruffins, S., \& Wiss, F. C. (1990c). Object relations in borderline adolescents. Journal of the American Academy of Child and Adolescent Psychiatry, 29, 338-348.

Westen, D., Ludolph, P., Silk, K., Kellam, A., Gold, L., \& Lohr, N. (1990d). Object relations in borderline adolescents and adults: Developmental differences. Adolescent Psychiatry, 17, 360-384.

Whalen, P. J., Bush, G., McNally, R. J., Wilhelm, S., Mclnerney, S. C., Jenike, M. A., \& Rauch, S. L. (1998). The emotional counting stroop paradigm: A functional magnetic resonance imaging probe of the anterior cingulate affective division. Society of Biological Psychiatry, 44, 1219-1228.

Widinger, T. A., \& Weissman, M. W. (1991). Epidemiology of borderline personality disorder. Hospital and Community Psychiatry, 42, 1015-1021.

Wilcox, R. R. (1997). Introduction to robust estimation and hypothesis testing. San Diego, CA: Academic Press.

Williams, J. M. G., Watts, F. N., MacLeod, C., \& Mathews, A. (1997). Cognitive psychology and emotional disorders. second edition. Chichester, England: John Wiley \& Sons.

Wilson, J., \& Costner, K. (1990). Dances with wolves [Motion Picture]. United States: Metro Goldwyn Mayer.

World Health Organization. (1991). World Health Organization: Tenth revision of the international classification of diseases, chapter $V$ (F: Mental and behavioural disorders. diagnostic criteria for research. (First ed.). Geneva: World Health Organization.

World Health Organization. (1993). World Health Organization: Tenth revision of the international classification of diseases, chapter V (F: Mental and behavioural disorders. diagnostic criteria for research. (Second ed.). Geneva: World Health Organization.

Young, J. E., Klosko, J., \& Weishaar, M. E. (2003). Schema therapy: A practitioner's guide. New York: The Guilford Press. 


\section{BIBLIOGRAPHY}

Zanarini, M. C. (1997). Role of sexual abuse in the etiology of borderline personality disorder. Washington, DC: American Psychiatric Press.

Zanarini, M. C., Frankenburg, F. R., DeLuca, C. J., Hennen, J., Khera, G. S., \& Gunderson, J. G. (1998a). The pain of being borderline: dysphoric states specific to borderline personality disorder. Harvard Review Psychiatry, 6, 201-207.

Zanarini, M. C., Frankenburg, F. R., Dubo, E. D., Sickel, A. E., Trikha, A., A., L., \& Reynolds, V. (1998b). Axis I comorbidity of borderline personality disorder. American Journal of Psychiatry, 155, 1733-1739.

Zanarini, M. C., Frankenburg, F. R., Dubo, E. D., Sickel, A. E., Trikha, A., A., L., \& Reynolds, V. (1998c). Axis II comorbidity of borderline personality disorder. Comprehensive Psychiatry, 39, 296-302.

Zanarini, M. C., Frankenburg, F. R., Reich, D. B., Marino, M. F., Lewis, R. E., Williams, A. A., \& Khera, G. S. (2000). Biparental failure in the childhood experiences of borderline patients. Journal of Personality Disorders, 14, 264-273.

Zimmerman, M., \& Mattia, J. I. (1999). Axis I diagnostic comorbidity and borderline personality disorder. Comprehensive Psychiatry, 40, 245-252. 
Samenvatting 


\section{SAMENVATTING}

De pathologie behorende bij de borderline persoonlijkheidsstoornis (BPS) is aan het begin van de twintigste eeuw afgegrensd van schizofrenie. In 1980 werd BPS formeel opgenomen in de psychiatrische diagnostiek, als één van de instabiele en emotionele (cluster B) persoonlijkheidsstoornissen (American Psychiatric Association, 1980). In tegenstelling tot patiënten met schizofrenie hebben personen met BPS een overwegend intacte realiteitstoetsing; hun lijdensdruk is echter nauwelijks geringer. BPS wordt gekenmerkt door frequente, extreem negatieve affecten (vooral extreme angst en boosheid), ernstige destructieve neigingen (waaronder middelenmisbruik, agressieve uitbarstingen en automutilatie), gefragmenteerde of gedissocieerde belevingen ((Zanarini et al., 1998a) en een hoog suïciderisico (10\%; Paris, 1993). Patiënten met BPS rapporteren dan ook een grote vermindering van kwaliteit van leven (e.g., van Asselt et al., 2008). BPS is verder een relatief courante stoornis, met prevalenties van 1 tot $2 \%$ onder de algemene bevolking, en 10 en $20 \%$ onder respectievelijk ambulante en opgenomen patiëntenpopulaties (American Psychiatric Association, 1994, 2000; Samuels et al., 2002; Torgersen et al., 2001), en wordt in drie van de vier gevallen bij vrouwen aangetroffen (American Psychiatric Association, 2000; Paris, 2004; Widinger \& Weissman, 1991).

Clinici en onderzoekers hebben sinds het ontstaan van BPS geprobeerd grip op deze complexe stoornis te krijgen. Zij formuleerden daartoe vele theorieën en hypothesen, maar lieten empirische toetsing hiervan veelal achterwege. Dit proefschrift beoogt bij te dragen aan het begrip van BPS middels empirische toetsing van een aantal van deze ideeën. Een terugkerende algemene kwestie is de vraag of BPS fundamenteel anders is als andere stoornissen. Het proefschrift concentreert zich daarbij op twee kenmerken die volgens hedendaagse psychologische theorieën een centrale rol spelen in BPS: hypervigilantie en extreme evaluaties.

Hypervigilantie kan worden begrepen als een angstige toestand, waarin een individu de aandacht selectief op bedreigende, vooral op met gevaar geassocieerde, stimuli richt. Initiële automatische codering van bedreigende stimuli krijgt in deze modus voorrang boven strategische herhaling van het materiaal voor opslag in het geheugen. Enkele theorieën stellen dat het gedrag van patiënten met BPS grotendeels voortkomt uit paniek en wanhoop, en de hiermee gepaard gaande hypervigilantie. Modellen geïnspireerd op de ideeën van Aaron Beck (e.g., Beck, 1979) veronderstellen dat BPS zich onderscheidt door drie cognitieve basisschema's, i.e.: 'Ik ben machteloos en kwetsbaar', 'Ik ben inherent onacceptabel' en 'Anderen zijn / de wereld is gevaarlijk en kwaadwil- 
lend'. Deze schema's zijn slecht verenigbaar (toenadering en vermijding van anderen) en genereren in geactiveerde toestand excessieve angst en hypervigilantie (Pretzer, 1990). Traumatheorieën, gebaseerd op vele rapportages van verwaarlozing en misbruik in de jeugd onder personen met BPS (Herman et al., 1989; Sabo, 1997; Zanarini, 1997), conceptualiseren BPS als posttraumatische stoornis met automatische selectieve aandacht voor en strategische vermijding van traumagerelateerde stimuli. Het model voor BPS van Marsha Linehan (1993) tenslotte veronderstelt dat een fundamentele emotionele disregulatie, onder meer bestaande uit een bijzonder hoge sensitiviteit voor emotionele stimuli in het algemeen, centraal staat in BPS. Emotionele disregulatie zou voortkomen uit een aangeboren emotioneel temperament gecombineerd met een invaliderende opvoeding.

Een tweede kenmerk van BPS dat in dit proefschrift werd onderzocht is de neiging tot het maken van extreme evaluaties. Verscheidene theorieën veronderstellen dat de onstabiele en extreme affecten en gedragingen van patiënten met BPS (mede) het gevolg zijn van de neiging anderen en/of de wereld in het algemeen in extreme termen te evalueren. Beckiaanse modellen (e.g., Pretzer, 1990; Layden et al., 1993) postuleren twee typen extreme evaluaties in BPS: 'dichotoom denken' en schemacongruente evaluaties. Dichotoom denken wordt gedefinieerd als:

"de neiging ervaringen te evalueren in termen van elkaar wederzijds uitsluitende categorieën (e.g., goed of slecht, succes of mislukking, betrouwbaar of onbetrouwbaar) in plaats van ervaringen langs een continuüm te plaatsen"

(Pretzer, 1990, p. 187). Evaluaties congruent met de eerder beschreven basisschema's worden, vanwege hun onverenigbaarheid, eveneens verondersteld personen met BPS uit evenwicht te brengen. Psychoanalytische objectrelatietheorieën conceptualiseren extreme evaluaties als 'splijting': een primitief afweermechanisme, dat goede en slechte aspecten van zelf- en ander-representaties gescheiden houdt ter voorkoming van de vernietiging van goede door slechte aspecten. In het model van Otto Kernberg (1996) representeert dit afweermechanisme een cognitief-emotioneel defect dat BPS fundamenteel onderscheidt van 'neurotische' stoornissen (zoals angst- en depressieve stoornissen). Extreme evaluaties kunnen tenslotte voortkomen uit een algemene negativistische cognitieve stijl, die in een aantal empirische studies specifiek bleek voor BPS, maar waarvoor nog geen theoretische verklaring bestaat. 
Dit proefschnft bevat viff experimenten die hypervigilantie en extreme evaluaties in BPS onderzochten aan de hand van de volgende onderzoeksvragen:

- Kenmerkt BPS zich door hypervigilantie en extreme evaluaties?

- Op welke stimuli richt zich hypervigilantie in BPS en welke extreme evaluaties typeren BPS?

- Welke factoren zyn mogelijke voorspellers voor hypervigilantie en extreme evaluaties in BPS?

- Hangt een geslaagde behandeling van BPS samen met verminderde hy" perviglantie en minder extreme evaluaties?

Een eerste experiment (zie hoofdstuk 2) toetste of BPS zich kenmerkt door hypervigilantie. of deze is gericht op schemagerelateerde stimuli en samenhangt met kindertrauma's en schema's. Verder werden samenhangen tussen hypervigilantie en BPS-symptomen geëxploreerd. Hiertoe werden 16 patiënten met BPS vergeleken met 18 patiënten met een cluster $C$ (angstige) persoonlijkheidsstoornis, 16 patiënten met een as-1 stoornis en 16 gezonde proefpersonen. Hypervigilantie werd gemeten met een emotionele Stroop taak met schemagerelateerde en -ongerelateerde, emotioneel negatieve en positieve, supraen subliminale persoongerelateerde stimuli. De aanwezigheid en ernst van kindertrauma's werd vastgesteld met de Vragenlijst Belastende Gebeurtenissen (VBG; van den Bossche et al., 1999). schema's en BPS-symptomen werden gemeten met achtereenvolgens de subschaal voor BPS van de Personality Disorder Belief Questionnaire (PDBQ-120; Dreessen \& Arntz, 1995) en de BPD Klachtenlijst (Arntz \& Dreessen, 1992).

Een tweede experiment (zie hoofdstuk 3 ) toetste wederom of BPS zich kenmerkt door hypervigilantie en of deze gericht is op schemagerelateerde stimuli (replicatie). Verder richtte dit onderzoek zich op de vraag of hypervigilantie bij BPS kan worden beînvloed door succesvolle psychotherapie en of verminderde hypervigilantie samenhangt met symptoomreductie. Het experiment vergeleek (1) 24 patiënten met BPS en 23 gezonde proefpersonen, alsmede (2) 16 patiënten met BPS bij het begin en eind van een intensieve, drie jaar durende psychotherapie, i.e., schemagerichte therapie (Young et al., 2003; Arntz, 2004) of overdrachtgerichte (transference-focused) psychotherapie (Clarkin et al., 1999). Hypervigilantie werd vastgesteld met een emotionele Stroop taak met schemagerelateerde en -ongerelateerde, emotioneel negatieve, supra- en su- 
bliminale persoongerelateerde stimuli. Symptoomniveau werd bepaald met de BPD-Klachtenlijst (Arntz \& Dreessen, 1992).

De resultaten van deze twee onderzoeken kunnen worden samengevat met de volgende antwoorden op de onderzoeksvragen betreffende hypervigilantie:

- BPS kenmerkt zich door hypervigilantie. Patiënten met BPS lieten meer hypervigilantie (voor bepaalde stimuli) zien dan patiënten met een as-I of cluster $C$ persoonlijkheidsstoornis en gezonde proefpersonen. Verder bleek de relatie tussen de aanwezigheid van BPS en hypervigilantie onafhankelijk van comorbide angst en depressieve stoornissen en comorbide cluster $\mathrm{C}$ persoonlijkheidsstoornissen.

- Hypervigilantie in BPS is niet eenduidig schemagerelateerd. Het eerste experiment vond meer selectieve aandacht bij patiënten met BPS dan bij de controlegroepen voor schemagerelateerde, emotioneel negatieve, supraliminale stimuli en alle emotioneel positieve, supraliminale stimuli. Daarnaast werd een trend gevonden voor een aandachtsbias voor schemagerelateerde, emotioneel negatieve, subliminale stimuli bij patiënten met BPS. Het tweede experiment vond geen effect voor schemagerelateerdheid: patiënten met BPS toonden in dit experiment een aandachtsbias voor alle negatief emotionele, supraliminale stimuli.

- Hypervigilantie in BPS hangt samen BPS-symptomen en trauma's, maar op verschillende wijzen voor negatieve en positieve stimuli. Aandachtsbias voor negatieve (schemagerelateerde) stimuli werd voorspeld door BPS-gerelateerde angstsymptomen en sexueel misbruik in de kindertijd. Aandachtsbias voor positieve stimuli werd enkel voorspeld door BPSgerelateerde identiteitsproblemen.

- Een geslaagde behandeling van BPS hangt samen met een, tot op normaal niveau, gereduceerde hypervigilantie.

Een derde experiment (zie hoofdstuk 4) onderzocht de prominentie van drie typen extreme evaluaties in BPS in interpersoonlijke situaties: dichotome (gemixt extreem negatieve en positieve), gespleten (gepolariseerd negatieve of positieve) en negativistische interpersoonlijke evaluaties, en toetste tevens de schema- en traumagerelateerdheid van deze extreme evaluaties. Het experiment vergeleek hiertoe 18 patiënten met BPS met 19 patiënten met een cluster $C$ persoonlijkheidsstoornis en 17 gezonde proefpersonen. Extreme evaluaties werden vastgesteld aan de hand van beoordelingen van 16 personages 


\section{SAMENVATTING}

uit filmfragmenten op visuele analoog schalen (VASen). Deze beoordelingen werden omgerekend naar scores voor dichotoom denken (gemiddelde absolute extreemheid van de beoordelingen), splijting (gemiddelde niet-absolute extreemheid van de beoordelingen) en negatief denken (gemiddelde beoordeling). Schemagerelateerdheid werd gemanipuleerd door variatie van het filmthema (bijvoorbeeld: kind en boze moeder in misbruiksituatie, vrouw en man op huwelijksreis, ter dood veroordeelde, opgetogen wetenschappers) en van de schaalpolen (bijvoorbeeld: betrouwbaar - onbetrouwbaar, interessant - saai). Extreem denken scores werden gerelateerd aan de ernst van kindertrauma's, vastgesteld met de VBG (van den Bossche et al., 1999).

Een vierde experiment (zie hoofdstuk 5) onderzocht de prominentie van de drie typen extreme in BPS in niet-interpersoonlijke situaties en de schemagerelateerdheid hiervan. Participanten waren 24 patiënten met BPS, 16 patiënten met een anti-sociale persoonlijkheidsstoornis, 10 patiënten met een cluster $C$ persoonlijkheidsstoornis en 25 gezonde proefpersonen. Extreme evaluaties werden vastgesteld aan de hand van beoordelingen op VASen van een frustrerend en bevredigend computerspel en de proefpersonen zelf. De beoordelingen werden op dezelfde wijze als in het derde experiment naar scores voor dichotoom denken, splijting en negatief denken omgerekend. Schemagerelateerdheid werd weer gemanipuleerd door variatie van schaalpolen.

Een vijfde en laatste experiment (zie hoofdstuk 6) richtte zich op de kwestie welk type extreme interpersoonlijke evaluaties (dichotoom denken, splijting, negativistisch denken) het meest indicatief is voor BPS-pathologie en op de vraag of extreme evaluaties in personen met BPS afnemen tijdens psychotherapie. Onderzocht werden reducties in de drie typen extreme evaluaties en BPS-symptomen van 64 en 46 patiënten met BPS tijdens respectievelijk de eerste en tweede helft van een intensieve, drie jaar durende psychotherapie (zie beschrijving bij tweede experiment). Scores voor extreme evaluaties werden bij elk meetpunt met beoordelingen van 9-10 schemagerelateerde emotionele en schema-ongerelateerde neutrale filmpersonages bepaald zoals in het derde experiment. Ernst van BPS-symptomen werd weer gemeten met de BPDKlachtenlijst (Arntz \& Dreessen, 1992).

De resultaten van deze drie onderzoeken kunnen worden samengevat met de volgende antwoorden op de onderzoeksvragen betreffende extreme evaluaties:

- BPS kenmerkt zich door extreme (negativistische) evaluaties. Deze evaluaties waren specifiek voor patiënten met BPS vergeleken met personen 
met andere persoonlijkheidsstoornissen zoals cluster $C$ persoonlijkheidsstoornis en antisociale persoonlijkheidsstoornis.

- Vooral negativistische extreme evaluaties typeren BPS. Patiënten met BPS lieten niet duidelijk meer dichotoom denken en splijting zien dan controleproefpersonen. Wel werd een samenhang gevonden tussen reductie van BPS-symptomen en dichotoom denken. De negativistische evaluaties van patiënten met BPS waren niet eenduidig schema- of persoonsgerelateerd: zij beoordeelden ook schema-ongerelateerde en nietinterpersoonlijke stimuli negatiever dan personen met een andere persoonlijkheidsstoornis en gezonde proefpersonen.

- Een mogelijke voorspeller voor negativistische evaluaties in BPS is seksueel misbruik in de kindertijd. Dit type misbruik liet een positieve samenhang zien met negativistische evaluaties, maar alleen voor schema-gerelateerde negatieve personages.

- Symptoomreductie bij patiënten met BPS hing samen met verminderde extreme evaluaties, maar alleen verminderde dichotome evaluaties en met name tijdens de eerste therapiehelft. De dichotome evaluaties waren hierbij steeds 'multidimensioneel': deze bestonden altijd uit gemixt nega-tieve en positieve extreme evaluaties (bijvoorbeeld erg knap en erg kwaad-aardig) in plaats van gepolariseerd negatieve of positieve extreme evaluaties (bijvoorbeeld erg lelijk en erg kwaadaardig, of erg knap en erg goedaardig). Negativistische evaluaties namen juist toe bij afnemende BPS-symptomen tijdens de eerste therapiehelft en neigden pas tot afname bij afnemende symptomen (trend) tijdens de tweede therapiehelft.

Wat betekenen deze bevindingen voor de theoretische veronderstellingen betreffende BPS?

Hypervigilantie bleek een terugkerend kenmerk van BPS. Dit sluit aan bij eerder onderzoek naar hypervigilantie bij BPS (Arntz et al., 2000; Waller \& Button, 1999). Het past ook bij andere bevindingen die op een duidelijk angstige en hypervigilante kant van BPS wijzen, zoals (1) de hoge co-morbiditeit van BPS met angststoornissen en angstige (cluster $C$ ) persoonlijkheidsstoornissen (Zanarini et al., 1998b, 1998c; Zimmerman \& Mattia, 1999), (2) de hoge prevalentie van kindertrauma's in BPS (e.g., Sabo, 1997), en (3) angst-gerelateerde experimentele bevindingen patiënten met BPS zoals toegenomen activatie van de amygdala (Herpertz et al., 2001a) en genormaliseerde amygdala activatie 


\section{SAMENVATTING}

na een geslaagde behandeling (Arntz et al .2008b: Schnell \& Herpertz, 2007). Het conceptualiseren van BPS als angststoornis biedt een interessant nieuw perspectief voor onderzoek en klinische praktık. Een klinische implicatie is de zinvolheid van angst en trauma-interventies in de behandeling van BPS.

Hypervigilantie bij BPS bleek noch eendudig schemagerelateerd, noch een algemeen fenomeen. zoals voorspeld door achtereenvolgens het Beckiaanse model voor BPS (Pretzer, 1990) en de theorie van Linehan (1993). Deze bevindingen zetten vraagtekens bij schemacorrectie als behandeling voor hypervigilantie en bij een algemene in plaats van gerichte emotionele vaardigheidstraining. Hypervigilantie in BPS lijkt voorlopig het best te worden verklaard met het traumamodel gezien de bevinding dat selectieve aandacht bij patiënten met BPS, tenminste aandacht voor emotioneel negatieve stimuli, samenhing met angstsymptomen en sexueel misbruik in de kindertijd. Selectieve aandacht voor emotioneel positieve stimuli correleerde echter niet (positief) met BPSgerelateerde angstsymptomen en kindertrauma's, maar met BPS-gerelateerde identiteitsproblemen. Dit type hypervigilantie representeert mogelijk: (1) een appetitieve bias, (2) vermijding van negatieve stimuli of (3) splijting. Vervolgonderzoek met een taak die toenaderende en vermijdende aandacht onderscheidt, kan hierover uitsluitsel geven.

Dichotoom denken kenmerkte BPS in slechts één van de drie onderzoeken naar extreme evaluaties (zie hoofdstuk 6 versus hoofdstukken 4 en 5). Dit wijkt af van eerdere bevindingen met vergelijkbare paradigma's waarin proefpersonen filmpersonages (Veen \& Arntz, 2000) of hulpverleners (Ten Haaf \& Arntz, 2009) beoordeelden. Mogelijk is dichotoom denken afhankelijk van schemaactivatie, die gemakkelijker plaats vindt in een interpersoonlijke context (dan de niet-interpersoonlijke context van het vierde experiment; zie hoofdstuk 5) en bij ernstigere (borderline) persoonlijkheidsproblematiek (dan bij de middelmatig ernstige persoonlijkheidsproblematiek van de proefpersonen in het derde experiment; zie hoodtuk 4). Er kan ook sprake zijn geweest van (1) contaminatie door intelligentieverschillen in dit proefschrift (i.e., waarschijnlijk relatief lage intelligentie bij de controlegroepen in het derde experiment; zie hoofdstuk 4) en/of (2) contaminatie door intelligentie- en motivatieverschillen in eerder onderzoek (mogelijk hoge intelligentie en motivatie bij gezonde controlegroep van Veen and Arntz (2000)).

Dichotoom denken bij BPS lijkt het beste te worden geconceptualiseerd als denkfout zoals verondersteld in het Beckiaanse model van Pretzer (1990). De gemixt negatieve en positieve (i.e., multidimensionele) extreme evauaties in dit 
proefschrift en elders (Veen \& Arntz, 2000; Ten Haaf \& Arntz, 2009), en schemagerelateerde en -ongerelateerde extreme evaluaties duiden noch op splijting volgens het model van Kernberg (1996), noch op regressie naar een ander primitief cognitief-emotioneel ontwikkelingsniveau (Layden et al., 1993), noch op schemacongruent denken. De multidimensionele dichotome evaluaties zouden (een neiging tot) een genuanceerd oordeel en/of een verdraaide negativistische evaluatie kunnen representeren. Volgens Beck (1976) en Pretzer (1990) is dichotoom denken een dysfunctioneel fenomeen dat individuen kwetsbaar maakt voor depressiviteit, stemmingsschommelingen en interpersoonlijke problemen. De denkfout kan echter ook worden begrepen als een gevolg van stress (zie, Kroll, 1988), bijvoorbeeld als onderdeel van de 'primal' modus (zie, Beck, 1996) dat op overleving gerichte acties faciliteert en bij patiënten met BPS wellicht ook de spanningsregulatie dient, als tegenwicht voor gevoelens van leegheid. Voor therapeuten impliceren het multidimensioneel dichotoom denken en de aan symptoomreductie gerelateerde afname van dichotoom denken bij BPS dat deze evaluaties met voorzichtigheid gelabeld moeten worden en niet onbehandelbaar zijn.

Patiënten met BPS bleken zich in elk van de drie onderzoeken te onderscheiden door extremere negatieve evaluaties. Deze bevinding sluit aan bij klinische observaties van achterdochtigheid, slachtoffer-denken en de moeilijke therapeutische alliantie bij deze patiëntengroep (e.g., Kroll, 1988) en ook bij vele andere empirische studies naar negatieve interpersoonlijke en nietinterpersoonlijke evaluaties en affectieve reacties (Ackerman et al., 1999; Arntz \& Veen, 2001; Baker et al., 1992; Barnow et al., 2009; Benjamin \& Wonderlich, 1994; Kurtz \& Morey, 1998; Lerner \& St. Peter, 1984; Levine et al., 1997; Nigg et al., 1992; Renneberg et al., 2008; Rosenthal et al., 2005; Segal et al., 1992; Stein, 1996; Stern et al., 1997; Stuart et al., 1990; Wagner \& Linehan, 1999; Westen et al., 1990a, 1990b, 1990c, 1990d; Zanarini et al., 1998a). De relatieve persistentie of zelfs aanvankelijke toename van de negativistische evaluaties bij BPS gedurende langdurende, intensieve therapie is consistent met eerder onderzoek naar aanhoudend verminderd glimlachen na een emotionele crisis (Renneberg et al., 2008) en een gelijke mate van kwaadaardige interpersoonlijke evaluaties tussen adolescente en volwassen patiënten met BPS (Westen et al., 1990d). Voor de klinische praktijk betekent deze onderzoeksuitkomst dat reductie van negatief denken in BPS niet (direct) nodig en/of haalbaar, mogelijk zelfs contraproductief is.

De verklaring voor de negatieve evaluaties bij BPS-patiënten is niet duide- 


\section{SAMENVATTING}

lijk. De negatieve evaluaties bij BPS deden zich ook in schema-ongerelateerde situaties en vormen voor en lijken dus niet alleen te kunnen worden opgevat als schemacongruent denken. Speculatieve alternatieve oorzaken zijn: (1) een combinatie van negatieve affectiviteit en disinhibitie - deze omvat de capaciteit zichzelf te kalmeren, geworteld in een genetische kwetsbaarheid en verwaarlozende of misbruikende opvoeding (e.g., Trull, 2001), (2) traumatische en, moeilijker vast te stellen, subtraumatische ervaringen, en (3) dissociatie / afwezigheid.

Methodologische kanttekeningen bij de onderzoeken van dit proefschrift betreffen met name (a) de validiteit van de emotionele Stroop taak voor de vaststelling van selectieve aandacht en hypervigilantie, (b) de validiteit van de evaluatie-taak voor de vaststelling van dichotoom denken, splijting en negatief denken bij BPS en (c) de validiteit van de schema-gerelateerdheid en schemaactivatie. De onderzoeken van dit proefschrift brachten verder een drietal algemene methodologische aandachtspunten naar voren voor toekomstig experimenteel onderzoek bij patiënten met BPS: (1) disfunctionele interpersoonlijke schema's, (2) dissociatie / afwezigheid en (3) emotionele reactiviteit.

Afsluitend kan worden opgemerkt dat het onderzoek van dit proefschrift weinig aanwijzingen levert voor een fundamenteel verschil tussen BPS en andere (as-l) stoornissen, zoals verondersteld door objectrelatietheorieëen (i.e., primitieve persoonlijkheidsorganisatie) en enkele hedendaagse theorieëen (e.g., defectieve emotionele disregulatie). Patiënten met BPS blijken niet op een primitief cognitief-affectief niveau te functioneren (denken bijvoorbeeld multidimensioneel), delen belangrijke kenmerken met andere (neurotische) patiënten (schema-, angst- en traumagerelateerde hypervigilantie), en kunnen genezen van fundamenteel geachte problemen zoals hypervigilantie. Misschien wordt BPS geplaagd door mythen. Verder onderzoek is nodig om deze te ontkrachten. Dit proefschrift draagt daartoe bij. 
Dankwoord 


\section{DANKWOORD}

Arnoud Arntz, mijn promotor en copromotor. Heel veel dank voor jouw grote toewijding, vertrouwen en kameraadschap, de integere wetenschap die je aanreikte en al het andere.

Franciska Bos. Caroline van den Bossche. Katlijn van den Broek. Kristel van Gisbergen. Veerle Klinckenberg. Eveline Lenders. Jill Lobbestael. Ingrid Mertens. Rob Olij. Joke Opdenacker. Sjoerd Salet. Paula Smits. Hester Vlaanderen. Lieven Wachters. Marionne Wolfis. Dank voor jullie grote inzet en bijdragen.

De vele bereidwillige en hulpvaardige mensen in de instellingen waar de onderzoeken zijn uitgevoerd. Philippe Jacques, Han Keysers, Thamare van Roosmalen en eerder ook Anja Wassenberg van de RIAGG Maastricht. Ineke Brand van PMS Vijverdal in Maastricht. Monique Burger, Roos Jeulink, Marjon Nadort en Noor Tromp van GGZ Buitenamstel in Amsterdam. Manon van Boekel, Michael Bosch, Edith Duermeijer, Chantal van Duijn, Jeantine Harthoorn en Ismay Kremers van Universiteit Leiden. Gerrie Peters van GGz Eindhoven. Stefaan Vertommen van UC Sint Jozef in Kortenberg. An Hauwaert van MC Sint Jozef in Bilzen en CGG in Hasselt. Dirk van de Putte van PCZ in SintTruiden. Bruno de Keirsschieter van de penitentiaire instelling in Brugge. Ed Berretty en Kees Korrelboom van Parnassia PC in den Haag. Daan Jonker en Andre Huijsman van de Robert Fleury Stichting in Leidschendam. Jacques Oomen van het Vincent van Gogh Instituut in Venray. Wim Snellen van Altrecht in Zeist. Ab Hesselink en Roel Verheul van PC De Viersprong in Halsteren. Alle anderen.

Collega's van (het vroegere) DMKEP en de RIAGG Maastricht die direct of indirect hebben bijgedragen aan dit proefschrift. Susan Bögels. Tom Brinckman. Elsa Charles. Lindy Dullens. Hannie van Genderen. Bert Hoekzema. Marcel van den Hout. Rosanne Janssen. Peter de Jong. Merel Kindt. Noël Klinkers. Harmanne Menkveld. Madelon Peters. Annie Raven. Steph Sallaerts. Erik Schouten. Angela Verweij. Johan Vlaeyen. Anoek Weertman. Titia Weiland. Paul Wijts. Al die andere fijne collega's.

Babette Renneberg. Immer wieder warst du da, hast mir geholfen und mich inspiriert. Ganz herzlichen Dank! 
Jeffrey Young. Thank you for sharing your ideas and reflecting on those of mine.

Roger Borath. Rolf Sanderson. Corine van Sonsbeek. Bedankt voor het spandoek!

Urban Leim-Frübis. Thomas Heidenreich. Simone Saurgnani. Danke für eure liebe Unterstützung bei den letzten Schritten.

Heit en mem. Frits en Femke. Foar it paad hinne en altyd wer werom.

Theo van Aerts. Harrie Biessen. Josephine Giesen-Bloo. Frans Heuff. Lydia van der Louw. Martien Schrootten. De dames van de fietsclub. Andere vrienden. Zonder jullie zou het een stuk minder leuk zijn geweest!

Paranimfen Iris Engelhard en Sytske Tjeerdema, die steeds al aan mijn zijde waren.

Hans. Mijn allerbeste vriend, inspiratie, anti-depressivum, stuurman.

Natuurlijk alle patiënten die proefpersoon waren, wiens namen ik ook graag had genoemd.

Vele anderen, ook jullie bedankt! 


\section{Curriculum Vitae}

Simkje Sieswerda werd geboren op 5 februari 1971 te Marssum. In 1989 behaalde zij haar VWO-diploma aan het Titus Brandsma College te Bolsward. Tot 1993 studeerde zij exacte wetenschappen, onder meer resulterend in het propedeutisch examen Natuurkunde aan de Rijksuniversiteit Groningen. In 1994 en 1998 volgden het propedeutisch en doctoraal examen Psychologie aan de Universiteit van Amsterdam. Van 1999 tot 2005 werkte zij als Assistent in Opleiding aan de Universiteit Maastricht bij het Departement Medische, Klinische en Experimentele Psychologie en vanaf 2001 tevens als Psychotherapeut in Opleiding bij de RIAGG Maastricht, Afdeling Volwassenenzorg. In deze periode werden de studies uit deze dissertatie uitgevoerd en kwalificeerde zij zich tot Psychotherapeut BIG. Vanaf 2005 was zij werkzaam als Psychotherapeut bij de Verhaltenstherapie-Ambulanz van de Goethe Universität Frankfurt am Main. In 2007 verkreeg zij het volledig lidmaatschap van de Vereniging voor Gedragstherapie en Cognitieve Therapie. Sinds 2008 heeft zij een aanstelling als Akademische Mitarbeiterin bij het Psychologisches Institut van de Ruprecht Karls Universität Heidelberg en verricht zij onderzoek, psychotherapie en onderwijs. 